LA-5933-MS

Informal Report

UC-32 and UC-79

Reporting Date: March 1975

\section{FTRC $\varnothing$ L: A Computer Code to Group Collapse FTR Files and
tput Standard CCCC Interface Files Group Collapse FTR Files and
Output Standard CCCC Interface Files}

\author{
by \\ Patrick D. Soran \\ D. Soran
}

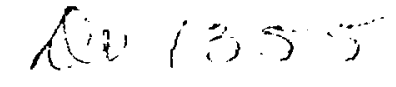

\author{
Issued: April 1975 \\ Issued: April 1975
}


In the interest of prompt distribution, this report was not edited by the Technical Information staff.

Printed in the United States of America. Available from National Technical Information Service

U.S. Department of Commerce

5285 Port Royal Road

Springfield, VA 22151

Price: Printed Copy $\$ 5.45$ Microfiche $\$ 2.25$

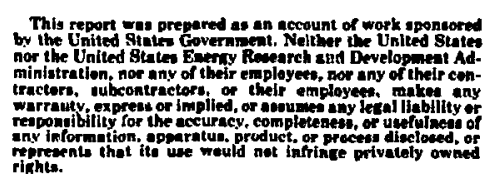


II. SOLUTION ALGORITHM AND VERIFICATION 2

A. Solution Method and Equations 2

B. Verification of FTRCOL 3

1. ISOTXS Verification 3

2. BRKoXS Verification 4

III. GUIDE TO USER'S APPLICATION

A. Overall Program Flow 4

B. Description of Input Data 5

1. Group Collapse Data 5

2. Output File Data 7

c. Output Description for a Test Problem 7

IV. PROGRAM INFORMATION

A. Program Structure 8

1. Role and Function of Subprograms 8

2. Program Flow 8

B. External and Internal Data Files 8

$\begin{array}{ll}\text { REFERENCES } & 10\end{array}$

$\begin{array}{ll}\text { APPENDIX A } & 10\end{array}$

FIGURES

FIg. 1. FTR calculational model . 4

Fig. 2. Simplified logical flow diagram for FTRCOL 5

F1g. 3. Amplified logical flow chart for FTRCOI. 9

TABLES

Table I. Equations employed in group collapsing 2

Table II. Library description 4

Table III. Reactor $k_{\text {eff values as a function of reactor model }}$ and cross
section library

Table IV, Function of subprograms $\quad 8$

Table V. Input/output units 9

This report was prepared

This report was prepared as an account of work

the United States nor States Government. INeither

Research and Development United States Energy

their employees, nor any of their nor any of

subcontractoss, or their empl their contractors,

warranty, express or their employees, makes any

liability or responsibility for the or assumes any legal

liability or responsibility for the accuracy, completeness 
FTRC $\emptyset$ L: A COMPUTER CODE TO GROUP COLLAPSE FTR FILES

AND OUTPUT STANDARD CCCC INTERFACE FILES

by

Patrick D. Soran

\section{ABSTRACT}

1. Program Identification: FTRCoL.

2. Computer for which Program is Designed: CDC-7600, CDC-6600.

3. Description of Function: FTRCDL takes multigroups cross sections and resonance self-shielding factors structured in an FTR format and collapses to fewer groups.

4. Method of solution: Cross-section information is collapsed by a $1 / \mathrm{E}, 1 / \mathrm{E}+\mathrm{fiss}$ ion, or an input weighting function.

5. Restrictions: Program is presently 1 inited to $100 \mathrm{fine} /$ broad groups, 3 temperatures, and $8 \sigma$ values. With minor modification these restrictions can be removed.

6. Running Time: Group collapsing from 30 groups to 5 groups of 15 isotopes using a $1 / E+$ fission spectrum and producing IS $\emptyset$ TXS and BRK@KS interface files requires 15 seconds of CDC-7600 time.

7. Unusual Features of the Program: FTRC $\phi \mathrm{L}$ produces version III standard IS $\emptyset \mathrm{TXS}$ and BRKGXS intexface files. The $1 / \mathrm{E}+$ fission spectrum has optional energy cut points and theta values and uses an adaptive integration technique to produce the fine group fluxes.

8. Machine Requirements: Two system input/output units and $s i x$ input/output units are required. All calculations a re performed and stored in small core.

9. Related Programs: FTRC $\emptyset \mathrm{L}$ can be used with the BINX and LINX for additional processing flexibility. Also the IS(j)XS and BRKDXS files can be converted back to FTR files by use of the FCFTR code.

10. Material Available: Source deck, test problems, and this report will be available from the Argonne Code Center. 
I. INTRODUCTION

FTRCOL is a computer code for group collapsing multigroup cross sections and resonance self-shielding factors (f-factors). The collapsed cross-section information is then given as ISOTXS ${ }^{1}$ and BRKOXs ${ }^{2}$ standard interface files. Input libraries must be structured in the BCD FTR ${ }^{3}$ library format. FTR 1ibraries are generally produced using the ETOX ${ }^{4}$ code and ENDF/B data, or one can produce ISOTXS and BRKOXS files by use of the FCFTR ${ }^{5}$ code. The need for a code such as FTRCOL arises from the requirement to perform two-dimensionai, Sn transport calculations using TWOTRAN-II ${ }^{6}$ (steady-state) or TWOTIME ${ }^{7}$ (timedependent) in a finite amounc of time, thus a need for few group libraries (in ISOTXS format). Additionally one may wish to consider resonance selfshielding and temperature effects in transport calculations directly, especially in safety codes where there is material motion and temperature gradients, thus a need for tew group f-factor librarles (in BRKOXS format).

\section{SOLUTION ALGORITHM AND VERIFICATION}

A. Solution Method and Equations

The FTRCOL code operates from a master library which exists in FTR format. Since the FTR format contains both information regarding multigroup neutron cross sections and resonance self-shielding factors only minimum additional information is required to produce ISOTXS and BRKOXS standard interface files. Given the master library and additional Intormation/isotope to be processed (see Sec.

III.B.) th: data on the master tape can be collapsed to a fewer number of multigroups using a number of flux weighting schemes. The flux (both the fine and broad grobs) terms are calculated or read in as prescribeo by the user.

Equations which are employed in the collapsing procedure ire specified in Table $I$. Using a fine group weigliting spectrum $\phi_{j}$, the $\phi_{j}$ terms are collapsed to bicoad group weighting spectrum. $\Phi_{j}$. Lower case subsicripts $i$ and $j$ Indicate fine groups and upper case stbscripts $I$ and $J$ represent collapsed cross section's and f-factors. The application of the equations given in Table $I$ is straightforward; however, a few words regarding the downscatter from group $\mathrm{J}$ to $\mathrm{J}+1$ might be appropriate. Jf one uses the group average $f$ lux to collapse the elastic

\section{TABLE I}

EQUATIONS EMPLOYED IN GROUP COLIAFSING

1. Capture, fission, inelastis, elastic, and total cross section

$$
\begin{aligned}
& \sigma_{J}=\sum_{j \in J} \sigma_{j}^{k} \Phi_{j} / \Phi_{j} \\
& \Phi_{j} \equiv \sum_{j \in J} \Phi_{j} \\
& \sigma_{j}^{k}=\text { the k-th type cross section for the } \\
& \phi_{j}=\text { the } j \text {-th group fine flux. }
\end{aligned}
$$

2. Total neutron yleld/fission (v)

$$
v_{j}=\sum_{j \in J} v_{j} \sigma_{j}^{f} \phi_{j} / \sum \sigma_{j}^{f} \phi_{j} .
$$

3. Average cosine of elastic scatzering angle ( $\mu$ )

$$
\begin{aligned}
\mu_{J} & =\sum_{j \in J} \mu_{j} \sigma_{j}^{s} \phi_{j} / \sum_{j \in J} \sigma_{j}^{s} \phi_{j} \\
\sigma_{j}^{s}= & \text { the elastic scattering cross section } \\
& \text { for the } j-t h \text { fine group. }
\end{aligned}
$$

4. Downscattering

$$
{ }^{C i} I \rightarrow J=\sum_{i \in I} \sum_{j=J} \sigma_{i \neq j}^{i n} \phi_{i} / \sum_{i \in J} \phi_{i}
$$

however,

$$
\begin{aligned}
& \sigma_{I-1} \rightarrow I=\sigma_{I-1}^{d}+\sigma_{I-1 \rightarrow I}^{i n} \\
& d=\underset{l}{\text { text }} \text { elastic downscatter (see discussion in } \\
& \text { in }=\text { Inelastic. }
\end{aligned}
$$

5. In-Group Scattering

$$
\sigma_{I \rightarrow I}=\sigma_{I}^{t r}-\sigma_{I}^{a}-\sum_{J=I+1} \sigma_{I-J}
$$

where

$$
\begin{aligned}
& \sigma_{I}^{t r}=\left(\sigma_{I}^{t}-\left(\sigma_{I}^{f}+\sigma_{I}^{c}+\sigma_{I}^{i n}\right)\right)\left(1-\mu_{I}\right) \\
&+\sigma_{I}^{f}+\sigma_{I}^{c}+\sigma_{I}^{i n} \\
& \sigma_{I}^{a}= \sigma_{I}^{f}+\sigma_{I}^{c}-\left[\sum \sigma_{I \rightarrow J}^{1 n}-\sigma_{I}^{i n}\right]^{*} \\
& \operatorname{tr}=\text { transport } \\
& t=\text { total } \\
& f=\text { fissinn } \\
& c=\text { capture }
\end{aligned}
$$

* The $(n, 2 n)$ cross section is multiplied by 2 and added to the inelastic scattering matrix; furthermore, the $(n, 2 n)$ cross section is subtracted from the capture (c) cress section. 
TABLE I (cont)

6. f-factor (capture, fission, elastic)

$$
f_{I}=\frac{\sigma_{I}\left(T, \sigma_{0}\right)}{\sigma_{I}\left(T_{\text {ref }}, \infty\right)},
$$

where

$$
\begin{aligned}
& \sigma_{I}\left(T, \sigma_{0}\right)=\sum_{i \in I} \sigma_{i} f_{i} \phi_{i} / \sum_{i \in I} f_{i} \phi_{i} \\
& \sigma_{I}(T \text { ref }, \infty)=\sigma_{I} \\
& T=\text { temperature }(K) \\
& \sigma_{0}=\text { discrete background cross sections. }
\end{aligned}
$$

scattering to the adjacent group appreciable errors can occur. 8,9 To clrcumvent this problem the lower boundary flux is used. Since elastic group transfer occurs near the lover group boundary the choice of the lower boundary for collapsing the elastic transfer is more logical than the group averaged flux. The method used to calculate elastic transfer is that of $1 \mathrm{DX}^{8}$ and is repeated in this report for convenience. For group $J$, the elastic transfer rate for each isotope is calculated using the flux at lethargy $\mathrm{U}_{\mathrm{J}}^{\mathrm{y}}$ such that

$$
\mathrm{U}_{\mathrm{J}}^{\prime}=\mathrm{U}_{\mathrm{J}}-0.66 \xi_{\mathrm{J}}
$$

where:

$\mathrm{U}_{\mathrm{J}}$ is the lethargy at lower group toundary $\mathrm{J}$, and $\xi_{\mathrm{J}}$ is the average lethargy change by elastic scattering for group $\mathrm{J}$.

Employing linear interpolation on the term $\xi \star^{e^{*} \phi} \phi(u)$ the downscattering is calculated as

$$
\sigma_{\mathrm{J} \rightarrow \mathrm{J}+1}=\frac{1}{\Delta \mathrm{U}_{\mathrm{J}}}\left[\left(\xi \sigma^{\mathrm{e}}\right)_{\mathrm{J}}+\frac{\mathrm{U}_{\mathrm{J}}^{\prime}-\mathrm{U}_{\mathrm{J}}}{\mathrm{U}_{\mathrm{J}+1}-\mathrm{U}_{\mathrm{J}}}\left[\frac{\phi\left(\mathrm{U}_{\mathrm{J}+1}\right)}{\phi\left(\mathrm{U}_{\mathrm{J}}\right)}\left(\xi \sigma^{\mathrm{e}}\right)_{\mathrm{J}+1}-\left(\xi \sigma^{\mathrm{e}}\right)_{\mathrm{J}}\right]\right]
$$

Equation (1) can be applied for every group $J$ except for $\mathrm{J}=1$.

Note that the collapse of the $f$-factors is performed by calculating a fine group shielded cross section at some temperature $(T)$ and background cross section $\left(\sigma_{0}\right)$ collapsing this shlelded cross section and dividing by the prevlously collapsed infinite dilution cross section. In FTRCOL, no special consideration is given to the collapse of the total and transport f-factor.

\section{B. Verification of FTRCOL}

Two objectives were sought in verifying the few group cross-sections information calculated by FTRCOL:

a. One which could check the ISOTXS file and

b. One which could check the ISOTXS and BRKOXS file.

Both files should be verified on a realistic descrip$t$ ton of a nuclear reactor. At this time only the ISOTXS file has been verified on a reactor model with the BRKOXS file verifled by analytic models.

\section{ISOTXS Verification}

Figure 1 represents an FTR calculational model (referred to as the detail model) and is discussed in Ref. 10. To reduce computational costs, the FTR model used in the present cross-section validation was that of Fig. 1 modified by using symetry about the midplane and eliminating the upper core structure (referred to as the modified model). This modification reduces computation time by a factor of eight for a 10-energy group, two-dimensional transport calculation, yet is sufficiently accurate as is discussed below.

The cross-section set employed in the analysis described in Ref. 10 was a 10-group set (referred to as the reference set) ${ }^{11}$ obiained by using flux volumes from a 30-group $\mathrm{MC}^{2}$ - generated set. ${ }^{12}$ (MC $^{2}$ problems were run with 30-energy group independent isotope data sets for the inner core, outer core, and axial reflector, and the flux volumes were calculated using the $\mathrm{MC}^{2}$-generated data on the detail model.) The reference library has temperature, energy, and spatial effects incorporated within tts 


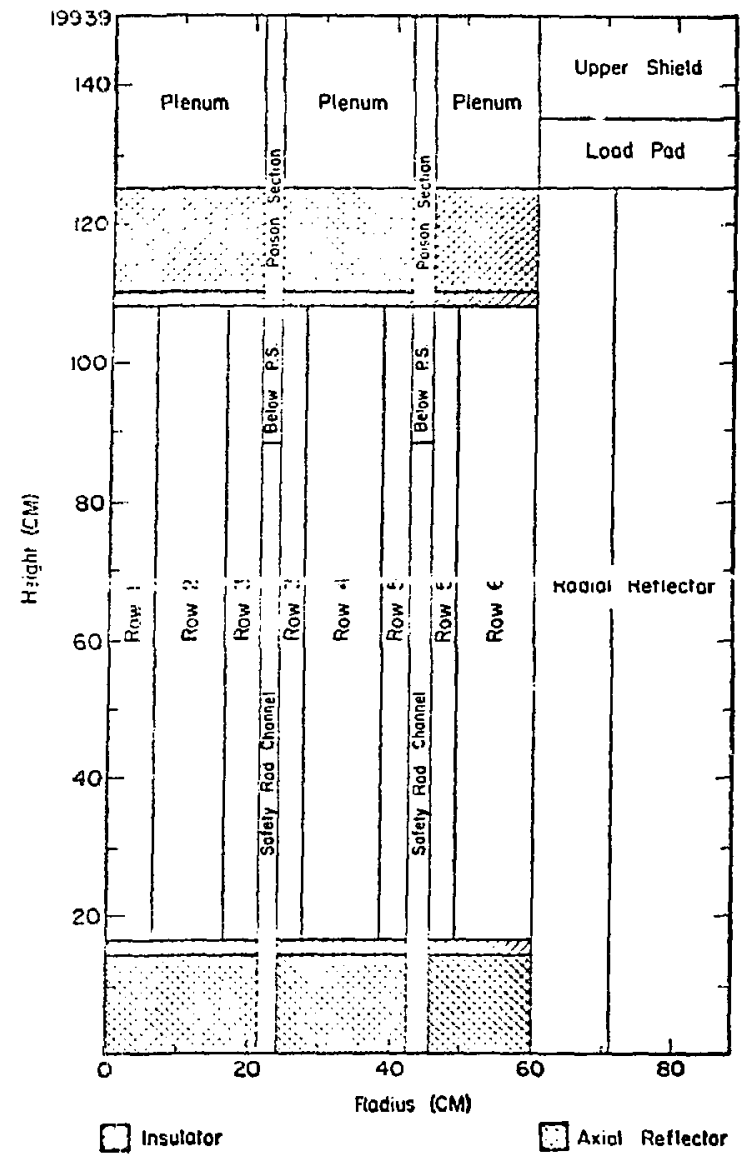

Fig. 1. FTR calculational model.

collapsing a 29-group library. ${ }^{13}$ (Sae Table II for more information on all of the libraries used in this study.)
Having the necessary ISoTxs libraries and calculational models, validation consisted of three steps: 1) detail model and the reference cross-section set, 2) modified model and the reference crosssection set, and 3 ) modified model and libraries $102 \mathrm{FO}, 102 \mathrm{E} 3,072 \mathrm{E} 3$, and 052E3. For each stage a $k_{\text {eff }}$ calculation was performed using TWOTRAN-II code with $r-z$ geometry and $S_{4}$ quadrature. The results of this study are iliustrated in Table III. It appears that the FTRCOL algorithm is sufficient for generation if ISOTXS information.

\section{BRKOXS Verification}

To date, there are no transport codes which use f-factors (BRKOXS files) directly in their calculation. Although TWOTRAN-II and TWOTIME are being modified to do the $f$-factor calculacion as an integral part of the code, modification is not complete. Consequently, only analytic models could be analyzed. To this end $f$-factors which were: a) constant, b) linear, and c) quadratic as a function of energy were collapsed by FTRCoL and compared to hand calculations. The agreement was excellent. Wher the modifications are complete to TWOTRAN-II, the BRKOXS file will be verified using the FTR calculational model discussed earlier.

III, GUIDE TO USER'S APPLICATION A. Overa11 Program Flow

A schematic flow chare for FTRCOL is given in

Fig. 2. A more detailed flow chart is provided in Sec. IV.A. 2 .

TABLE II

LIBRARY DESCRIPTION

\begin{tabular}{|c|c|c|c|c|c|c|}
\hline $\begin{array}{l}\text { Library } \\
\text { Name }\end{array}$ & $\begin{array}{l}\text { Point-Wise } \\
\text { Data Source }\end{array}$ & $\begin{array}{c}\text { No. of } \\
\text { Isotapes }\end{array}$ & $\begin{array}{l}\text { Chi value } \\
\text { Source }\end{array}$ & $\begin{array}{c}\text { No. of } \\
\text { Temp }\end{array}$ & $\begin{array}{l}\text { No. of } \\
\sigma_{0} \text { Values }\end{array}$ & F-Factors \\
\hline Reference ${ }^{a}$ & $\mathrm{ENDF} / \mathrm{B}-\mathrm{I}$ & 36 & ENDF/B-I & 1 & NA & No \\
\hline $102 \mathrm{~F} 0$ & $\mathrm{ENDF} / \mathrm{B}-\mathrm{II}^{\mathrm{b}}$ & 29 & Reference & 3 & 6 & Yes \\
\hline $102 \mathrm{E} 3$ & ENDE/B-III & 15 & Reference & 3 & 6 & Yes \\
\hline $072 E 3$ & ENDF/B-III & 15 & $\mathrm{ENDF} / \mathrm{B} \sim \mathrm{IV}$ & 3 & 6 & Yes \\
\hline $052 \mathrm{E} 3$ & $\mathrm{ENDF} / \mathrm{B}-\mathrm{III}$ & 15 & ENDF/B-IV & 3 & 6 & Yes \\
\hline
\end{tabular}

${ }^{a}$ See Ref. 5 for details.

${ }^{b}$ Moulfied to give agreement with various critical experiments. 
TABLE III

REACTOR $k_{e f} f$ VALUES AS A FUNCTION OF REACTOR MODEL AND CROSS-SECTION LIBRARY

\begin{tabular}{|c|c|c|c|c|c|}
\hline $\begin{array}{l}\text { Library } \\
\text { Name }\end{array}$ & $\begin{array}{c}\text { FTR } \\
\text { Model } \\
\end{array}$ & $\begin{array}{c}\text { No. of } \\
\text { Energy Groups }\end{array}$ & $k_{\text {eff }}$ Value & $\begin{array}{l}\text { Percent Dev. } \\
\text { from Reference }\end{array}$ & $\begin{array}{l}\text { cDC-7600 } \\
\text { Time (min.) }\end{array}$ \\
\hline Reference $^{a}$ & Detail $^{a}$ & 10 & 1.056 & & 62 \\
\hline Reference & Modified ${ }^{b}$ & 10 & 1.047 & 0.85 & 8.2 \\
\hline $102 \mathrm{FO}$ & Modified & 10 & 1.009 & 4.45 & 8.2 \\
\hline $102 \mathrm{E} 3$ & Modified & 10 & 1.020 & 3.41 & E. 2 \\
\hline 072E's & Modified & 7 & 1.030 & 2.46 & 5.4 \\
\hline $052 \mathrm{E} 3$ & Modified & 5 & 1.058 & -0.19 & 3.9 \\
\hline
\end{tabular}

See Ref. 5 for details.

b Same model as described in Ref. 5, but modified by using symetry about core midplane.

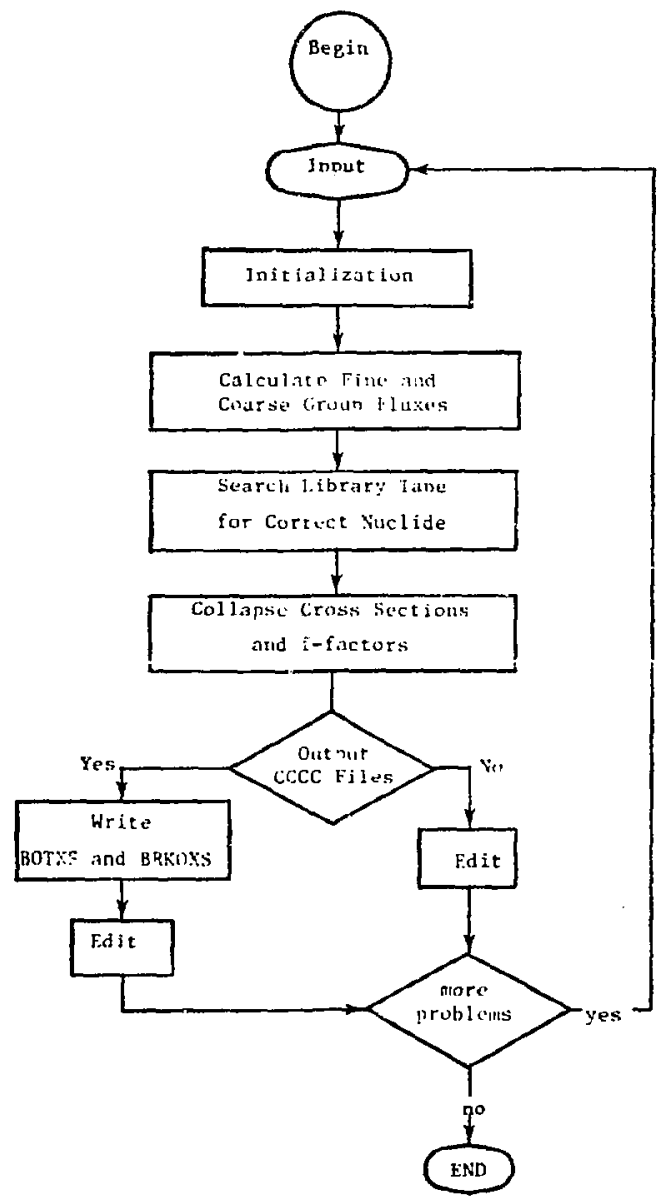

B. Description of Input Data

In the following pages the input data for FTRCOL are listed in exactly the order in which they are entered in the code. The data are divided into two categories: 1) data required to perform the group collapse, e.g., number of subgroups, isotopes to manipulate, and 2) data required to write the ISOTXS and BRKOXs files. The two sategories are explicitly discussed below.

\section{Group Collapse Data}

The user enters the data as noted below:

F1g. 7. Simplifled logical flow diagram for FTRCOL, 


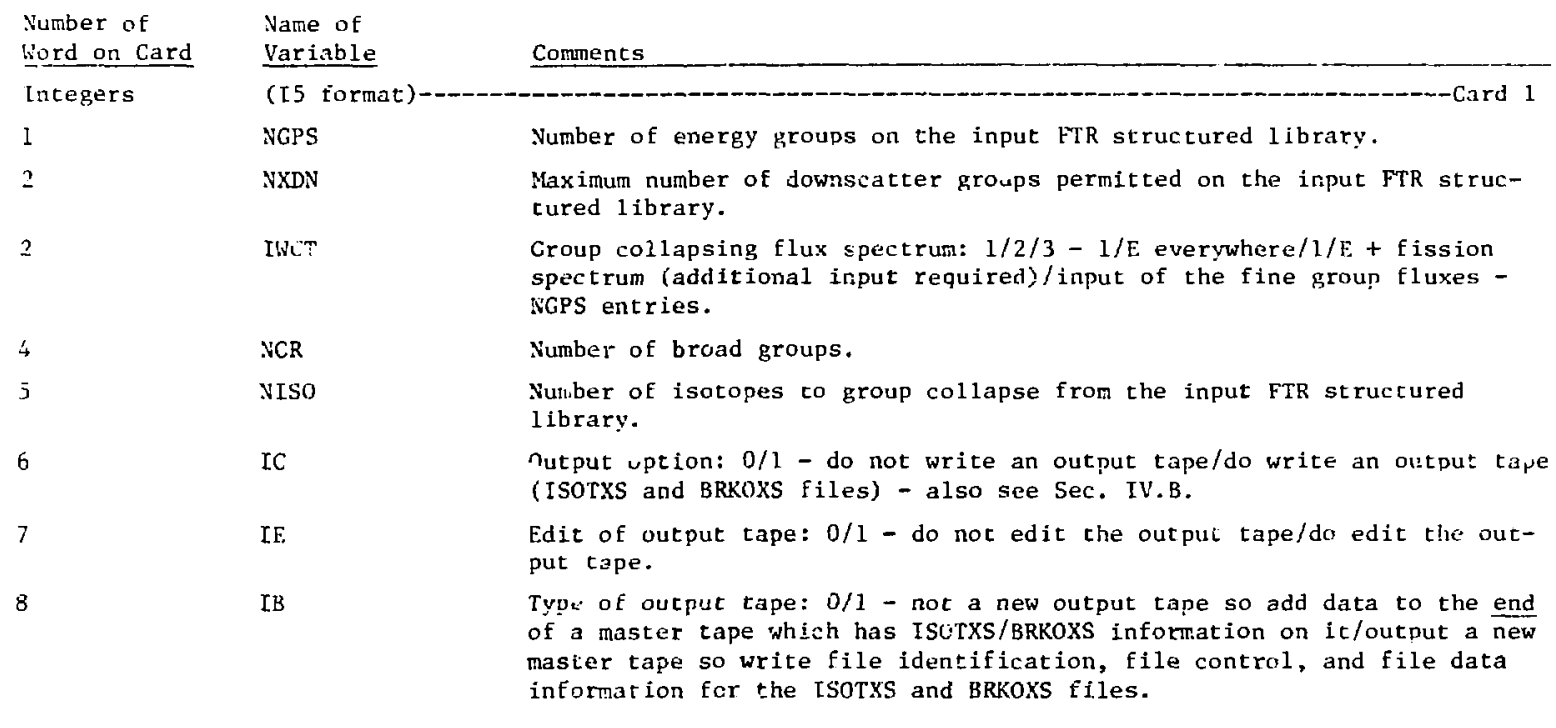

\section{If IWGT is ?}

Floating point (2E10 format)-

1 ECUT The energy cutoff point for the $1 / \mathrm{E}$ in units of $\mathrm{eV}$.

2 ETEMP Neutron cemperature used in the fission spectrum in units of eV.

If IWGT is 3

Floating point (7E10 format)- 2

1 The fine group fluxes which will be used in groups collapsing.

-

•

NGPS

IE IWGT is 1 or 2

Floating point ( 7 E10 format) -

- Card $2 / 3$

1

The actual energy (eV) cut points of the input FTR structured library. Need to calculate the broad group fluxes.

NCPS+1

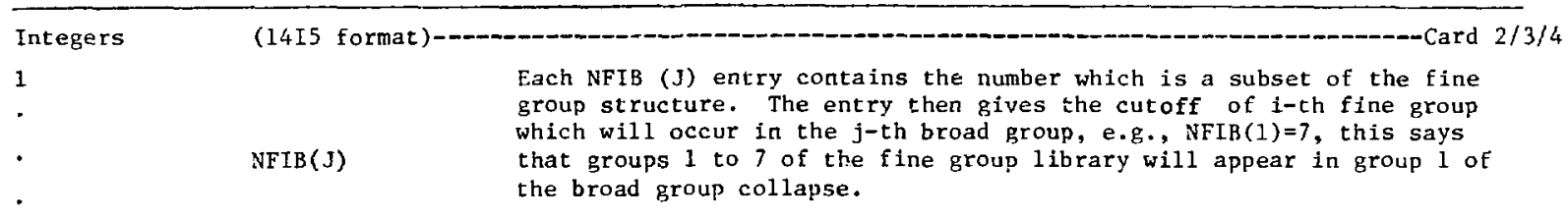

NCR 


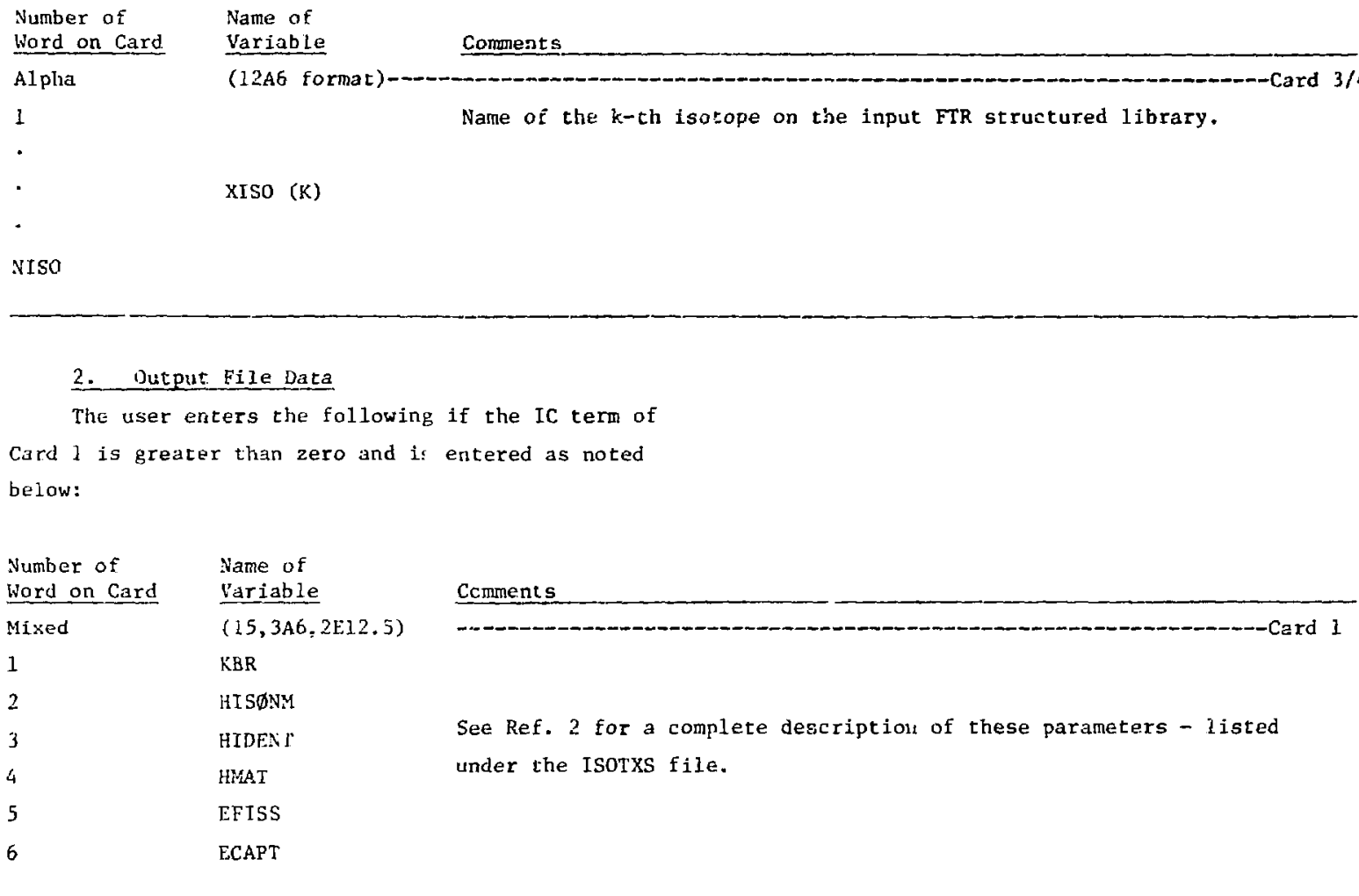

\section{If EFISS $>0$}

Floating point (6r.12.5)-

1

. - $\operatorname{cH} \emptyset(J) \quad T$ The isotope chi values for this isotope.

NCR

If $I B$ is 1 only

alpha $(12 \mathrm{~A} 6)$ card 3

1 Titie to go on the IsoTxs/BRKoxs file.

12

Cards 1 and 2 are repeated for NISO cases; note that Card 3 is only read in once if $I B$ is 1 . c. Output Description for a Test Problem The test problem presented in this report is given in Appendix A. The problem illustrated is that of generating 1 ibrary $052 \mathrm{E} 3$ as discussed in
Sec. II.B.1. The information given in Appendix A illustrates the input data for all isotopes and only gives the ISOTXS and BRKOXS information for one isotope although 15 isotopes were used in the original calculation. 
IV. PROGRAM INFORMATION

In this section we give some of the details of the FTRCOL program. The material contained in this section is designed to help in Euture local modification of the program. Additional information is provided by the program comment cards.

A. Program Structure

1. Role and Function of Subprograms

We describe in Table IV the function of all the subprograms in FTRCOL. In Table IV, the place Irom which each subprogram is called is also discussed.

\section{Program Flow}

In Fig. 3 we show an amplified version of Fig. 2 , depicting the overall flow of the FTRCOL program and showing subroutine names.

\section{B. External and Internal Data Files}

All files used for input, output, and scratch are referred to by symbolic names throughout the code. The user may easily change the physical unit assigned a file by modification of the unit name in subroutine UNITS. Also important to note, that for the present version of FTRCOL, if one wishes to generate output tapes, then the ISOTXS/BRKOXS files will appear on units $8 / 10$ if the NISO value is even. If NISO is odd, then the ISOTXS/BRKOXS files will appear on units $9 / 11$. Table $V$ indicates the files required by FTRCOL.

TABLE IV

FINCTION OF SUBPROGRAMS

\begin{tabular}{|c|c|}
\hline Siubroutine & Eunction \\
\hline $\begin{array}{l}\text { FTRCøL } \\
\text { INPU: }\end{array}$ & 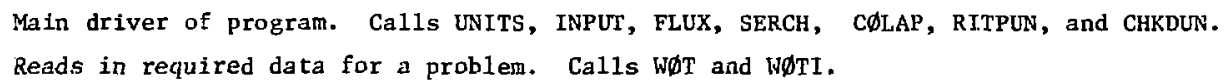 \\
\hline FLUX & Calculates broad and fine group fluxes used during collapse, Calls WWT and QNC7. \\
\hline CDEF & External function used to calculate $1 / \mathrm{E}+$ fissicn spectrum information. \\
\hline QNC7 & Adaptive integration routine. \\
\hline SERCi & Searches FTR tape for isotope of interest and processes. \\
\hline CØLAP & Collapses cross sectiun and f-factors. \\
\hline INITC & Initializes arrays. \\
\hline RITFUN & $\begin{array}{l}\text { Writes standard CCCC files - if desired, calls RITNW, RITIS } \emptyset \text {, and RITBRK. Additional } \\
\text { information is also read. }\end{array}$ \\
\hline RI'IS $\emptyset$ & Reads and arranges data for IS $\emptyset$ TXS-III file. Calls REED, RITE, and STфW. \\
\hline RITBRK & $\begin{array}{l}\text { Reads and arranges data for BRK } \emptyset \mathrm{XS}-\mathrm{III} \text { file. Calls REED, RITE, ST } \emptyset \mathrm{W}, \mathrm{C} \emptyset \mathrm{PYF} \text {, BF, and } \\
\text { XS. }\end{array}$ \\
\hline$B F$ & Stores f-factors in correct location for use in RITBRK. \\
\hline $\mathrm{xs}$ & Stores cross section in correct location for use in RITBRK. \\
\hline CHKDUN & Checks to see if all nuclides have been processed. Calls EDIT. \\
\hline EDIT & Will edit information if so desired. Galls BIS $\emptyset \mathrm{TX}$ and BzRKX. \\
\hline BISOTX & Reads and edits the ISøTXS-III file. Calls REED and WøT. \\
\hline BBR:XX & 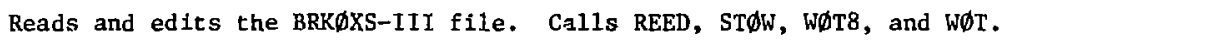 \\
\hline RITNW & $\begin{array}{l}\text { Produces the file identification, file control, and file data information for IS } \emptyset \text { TXS } \\
\text { and BRIKøXS. Calls FILD, ISFC, ISFD, BRIC, and BRFD. }\end{array}$ \\
\hline FILD & Produces file identification for ERK $\emptyset \mathrm{XS}$ and IS $\emptyset \mathrm{TXS}$. \\
\hline ISEC & Produces file control for ISpTXS. \\
\hline ISFD & Produces file data for IS $\emptyset \mathrm{TXS}$. Calls RITE. \\
\hline BRFC & Produces ftle control for BRK $\emptyset \mathrm{xS}$. \\
\hline BRFD & Produces file data for BRKøXS. Calls RITE. \\
\hline$\omega \emptyset \mathrm{T}$ & Prints a 1-, 2-, or 3-dimensional array. \\
\hline & Prints up to 8 one-dimensional arrays. \\
\hline
\end{tabular}


TABLE IV (cont)

Subroutine

WøTI

RITE

REED

STDW

TABLE $\mathrm{V}$

INPUT/OUTPUT UNITS

\begin{tabular}{lc} 
Name & $\begin{array}{c}\text { Logical } \\
\text { Unit }\end{array}$ \\
\cline { 2 - 2 } IU3 & 3 \\
IU4 & 4 \\
IU5 & 5 \\
IU6 & 6 \\
IU7 & 7 \\
IU8 & 8
\end{tabular}

IU9

9

IU10

\section{Function}

Prints a 1-, 2-, or 3-dimensional integer array.

Write binary file.

Reads uinary file.

Stores a vector.

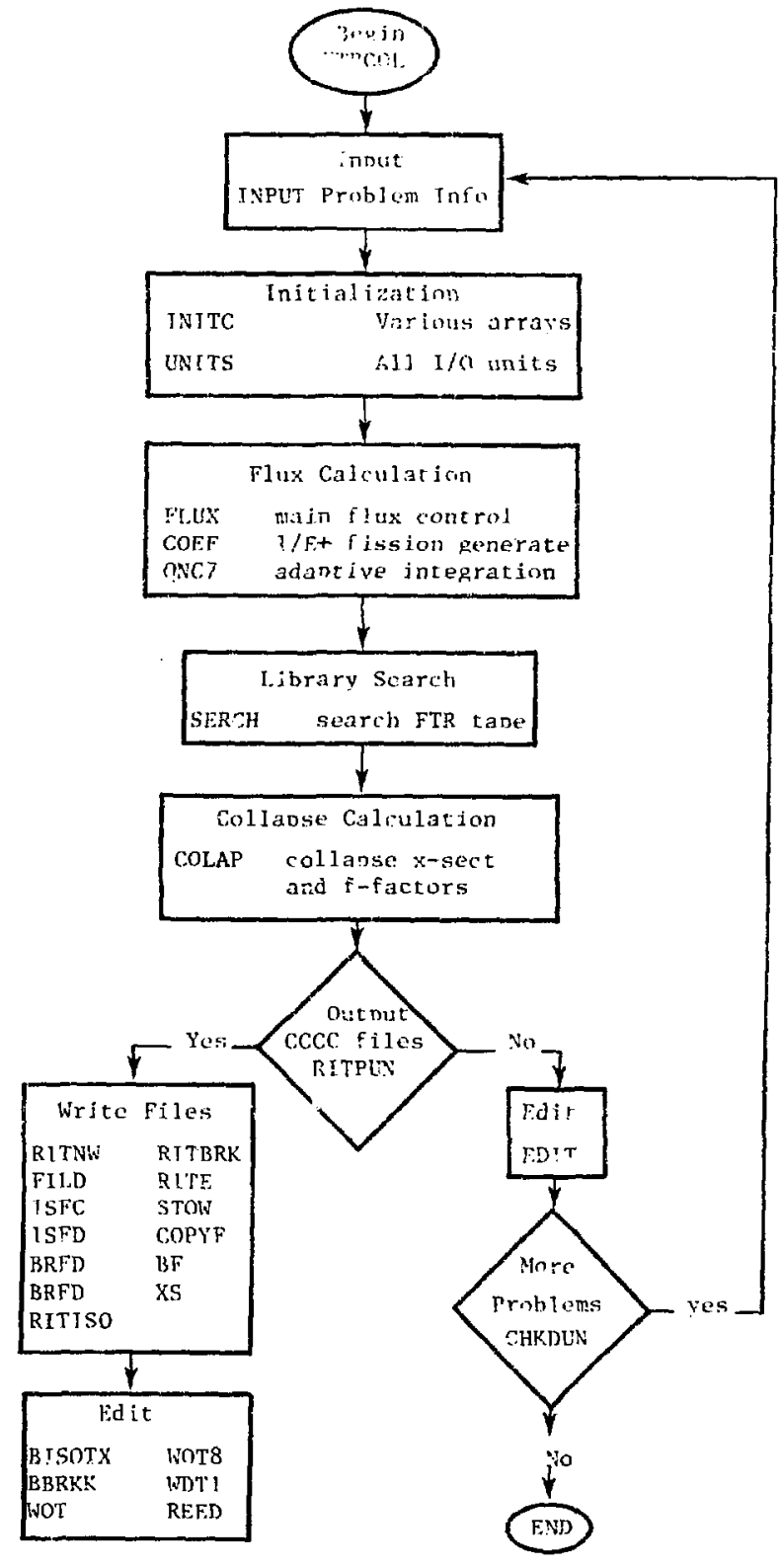

Fig. 3. Amplified logical flow chart for FTRCøL. 


\section{REFERFNCES}

1. B. M. Carmichael, "Standard Interface Files and Procedures for Reactor Physics Codes, Version III," Los Alamos Sclentific Laboratory report LA-5486-MS (February 1974).

2. Ibid.

3. R. E. Schenter, R. B. Kidmar, and J. V. Nelson, "FTR Set 300, MuItigroup Cross Section for FTR Design," Hanford Engineering Development Laboratory report HEDL-TME-71-153 (1971).

4. R. E. Schenter, J. L. Baker and R. B. Kedman, "ETOX, A Code to Calculate Group Constants for Nuclear Reactor Calculations," Battelle Northwest Laboratory report BNhL-1002 (May 1969).

5. P. D. Soran, "FCFTR - A Computer Code to Convert Scandard CCCC Incerface Files to FTR Files," Los Alamos Scientific Laboratcry report LA5745-MS (October 1974).

5. K. D. Lathrop and F. W. Brinkley, "TWOTRAN-II: An Interlaced, Exportable Version of the TWOTRAN Code for: Two-D1mensional Transport," Los Alamas Scientific Laboratory report LA-4848-MS (July 1973).

7. W. R. Reed, "TWOTIME - A Two-Dimensional Transport, Time-Dependent Code," Los Alanos Scientific Laboratory report, to be published.
8. R. W. Hardie and W. W. Little, Jr., "IDX - A One-Dimensional Diffusion Code Eor Generating Effective Nuclear Cross Sections," Battelle Memorial Institute, BNVL-954 (March 1969).

9. P. D. Soran, R. J. LaBauve, C. R. Wersbin, and J. S. Hendricks, "Multiplication Factor Dependence on Number of Muitigroups and Weighting Function Model," Am. Nuc. Trans. 17, November i973.

10. M. E. Battat, "Quarterly Report - Physics for Safety Anaiysis Program, April 1 through June 30, 1974," Los Alamos Scientific Laboratory report LA-5686-PR (July 1974).

11. Jay E. Boudreau and J. F. Jackson, "Recriticality Considerations in JMFBR Accidents," American Nuclear Soclety Fast Reactor Safety Meeting, April 2-4, 1974, Beverly lills, CA, USAEC CONF74041 .

12. B. J. Toppel, A. L. Rago, and D. M. O'Shea, "MC. ${ }^{2}$, A Code to Calculate Multigroup Cross Sections," Argonne National Laboratory report ANL-7318 (1967).

13. R. B. Kidman, Los Alamos Scientific Laboratory, private communication, November 1974.

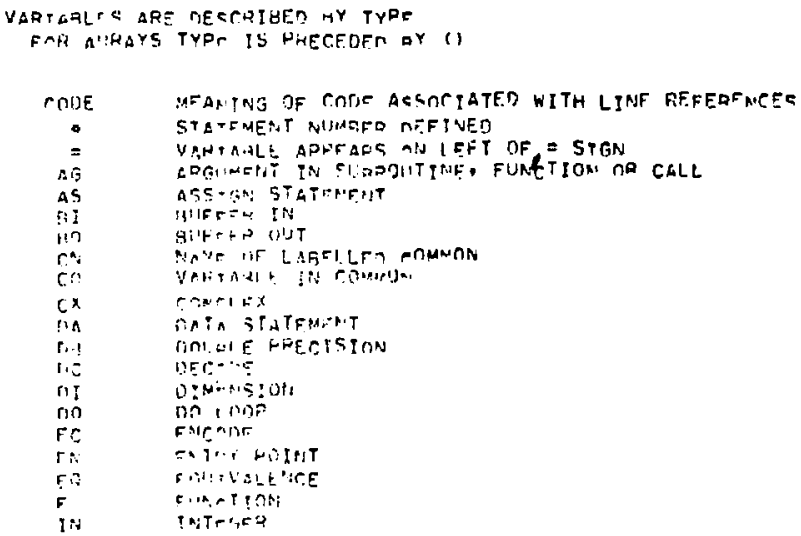

lathot

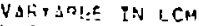

Leriticai.

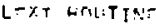

inter is?

abserth

Q1. T

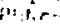

Dein

Din

Singi IN

$5: \Delta_{1}$

8*21 प्या

5.tonrift?

time la:

Tî. ALT

पराTF 


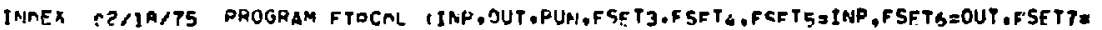

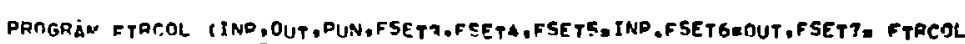

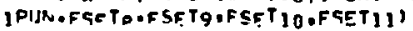

fotal

COLLAPSE nATA WHTH IS IN AN FTR TYPE FOQMAT TO FEWER GFOUPS FTRCOL

FTrets

COMAON Alingoos

FTHCal

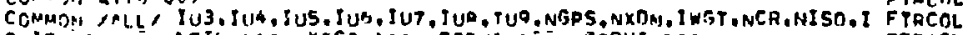

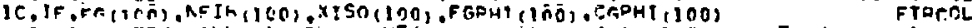

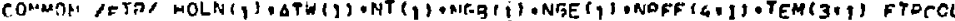

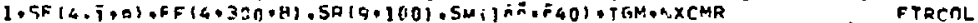

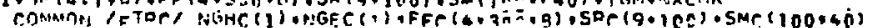

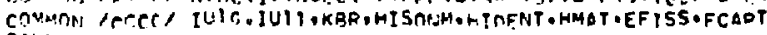

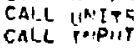

CAIL FIU

O CAII GFRMM INFIHOI

CALL TOLAP

CAI.L GTTOUN

CAIL CHKAIN INFSNOO

GO TO

FTRCOL
FTREOL

Ftacol
Ftaenl

FTPEOL

FTRCOL

FTRCOL

FTACOL

FTarol

ftactil

FTRCAL

ENi

$F$ TRCOL
FTACAL

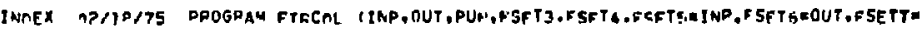

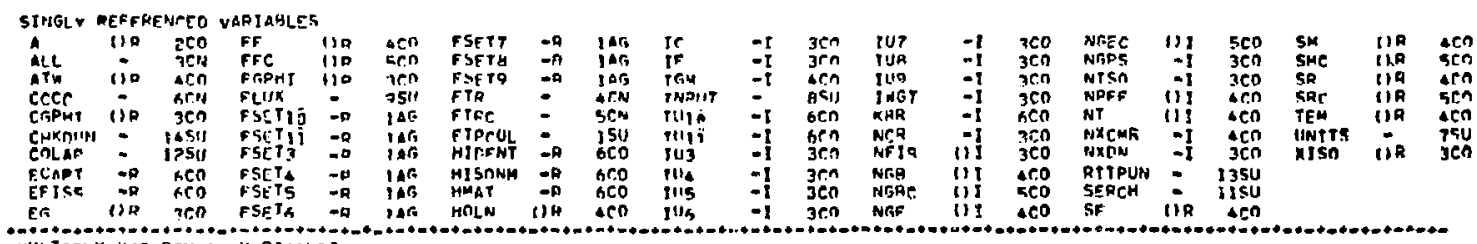

MULITDLYTHEFERTHE, VARTABLES

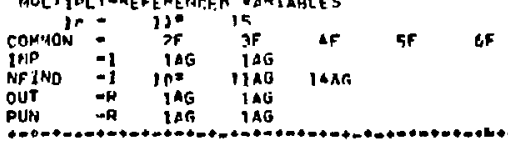

INAEX IPT/R/75

SURROLTINE UNITS

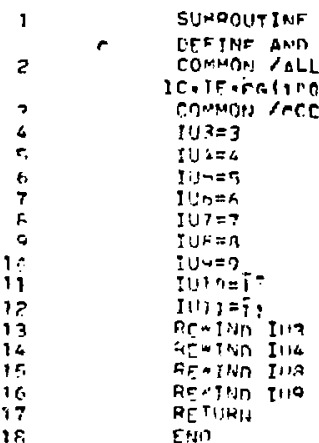




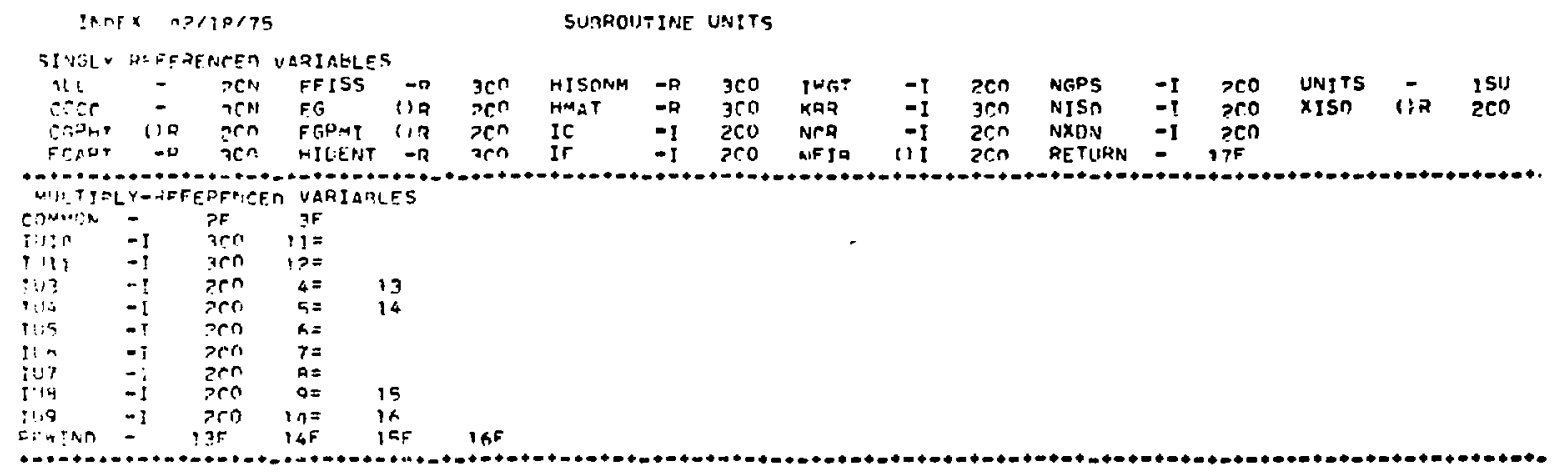

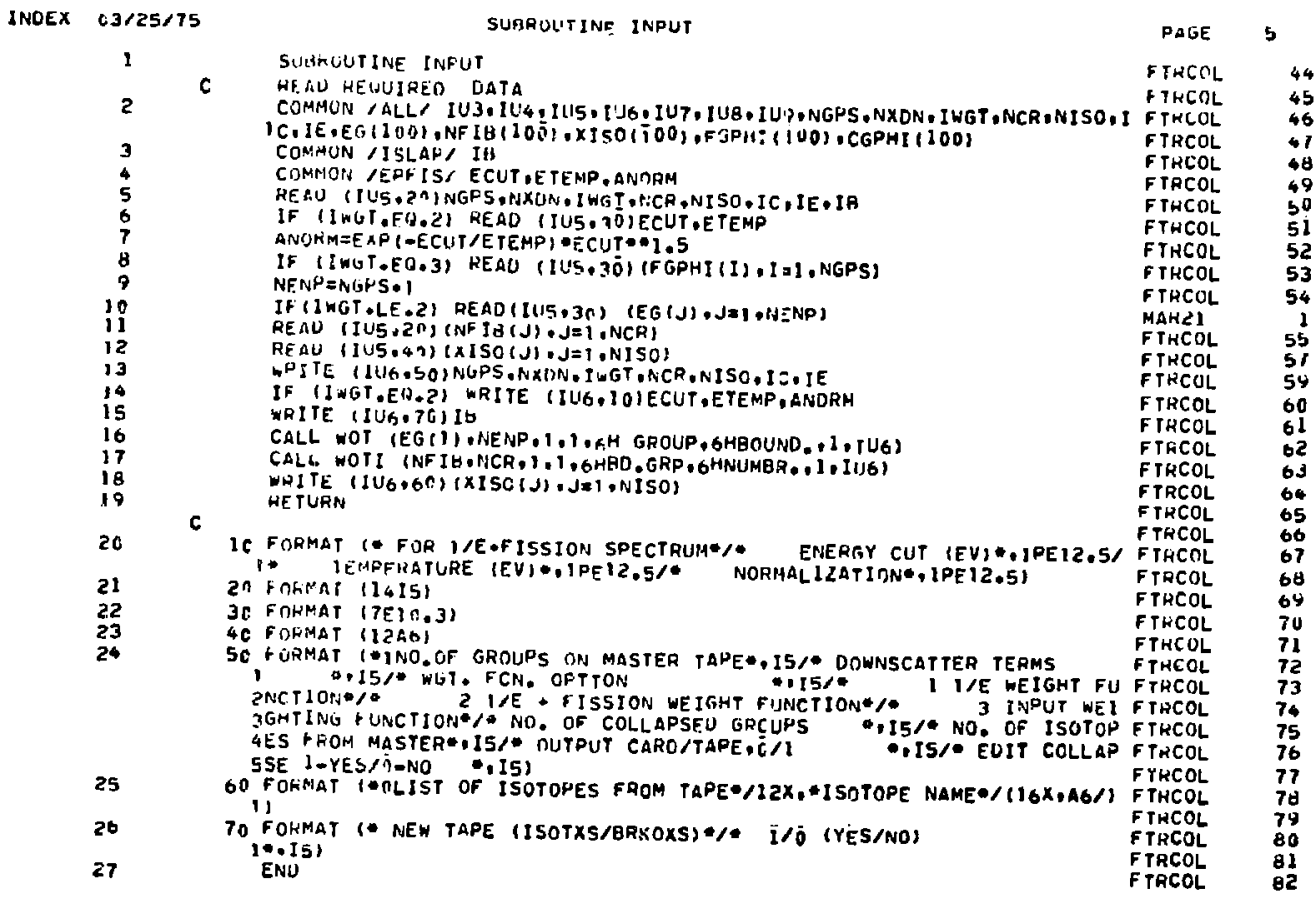




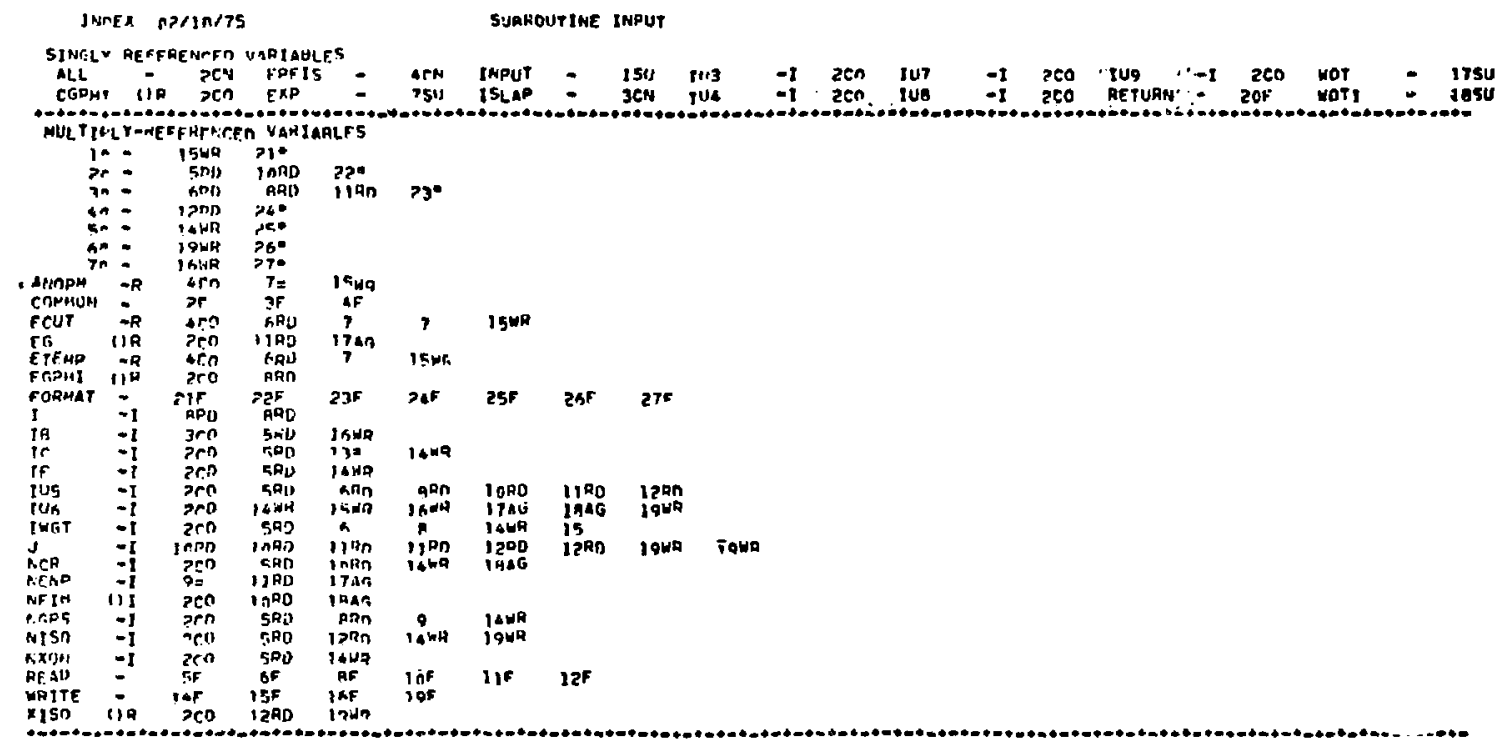

INREX O2/19/7S

SURROUTYNE FLUX

\begin{tabular}{|c|c|c|c|c|}
\hline$\frac{1}{3}$ & & $\begin{array}{l}\text { SUHDOUT IAFF FLUX } \\
\text { EXTERNAL CDEF }\end{array}$ & $\begin{array}{l}\text { FTRCSL } \\
\text { FTRCA: }\end{array}$ & $\begin{array}{l}83 \\
84 \\
85\end{array}$ \\
\hline 3 & $\mathbf{r}$ & 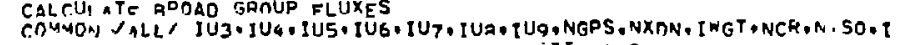 & $\begin{array}{l}\text { FTRCOL } \\
\text { FTRCOL }\end{array}$ & $\begin{array}{l}85 \\
86\end{array}$ \\
\hline 4 & & 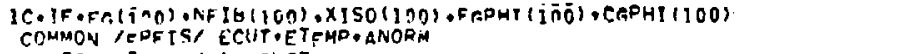 & $\begin{array}{l}\text { FTRCOL } \\
\text { FTRCOL }\end{array}$ & $\begin{array}{l}87 \\
\text { gi }\end{array}$ \\
\hline 5 & & 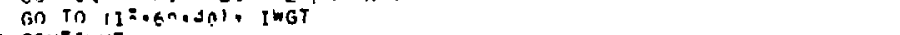 & FTRCOL & 89 \\
\hline$\frac{4}{3}$ & 10 & CONTtYIE & FTFCOL & 90 \\
\hline ? & & in $>$ i $i=, \cdot$ NirRs & FTRCAL & 91 \\
\hline & 20 & 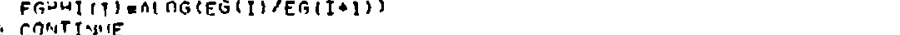 & FTPrOL & 92 \\
\hline 13 & 3c & Conthidie & FTECOL & 94 \\
\hline 1 & & WEITE IIIT.90) & FTACRL & 95 \\
\hline 17 & & 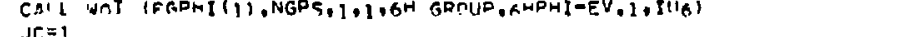 & FTRCAL & 96 \\
\hline$\%$ & & $J_{n} C_{i}=\bar{n} \quad t=\bar{i} \cdot$ NCR & $\begin{array}{ll}\text { atarol } \\
\text { FTh }\end{array}$ & 97 \\
\hline 15 & & $\operatorname{cog}+418)=n, a$ & FTACOL & 89 \\
\hline is & & $N x=\operatorname{ts} T \cap\{1+1$ & FTnenL & 100 \\
\hline 17 & & $13 n=\pi \quad 1=1 r_{0}, n x$ & FTirnt & 101 \\
\hline IR & & 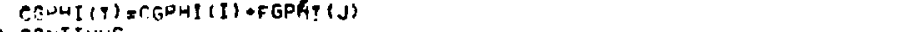 & FTRCOL & 192 \\
\hline 10 & 40 & CONTEMIE & FTRCOL & $\ln 3$ \\
\hline ?n & & $x=13 x+1$ & FTnC:L & 194 \\
\hline 21 & 50 & Contsinte & FTHROL & 105 \\
\hline $\begin{array}{ll}37 \\
33\end{array}$ & 60 & G. Tos 40 & FTACOL & 106 \\
\hline & r & OH- DUTH F + FISSION SPECTRUM & $\begin{array}{l}\text { FTFEOL } \\
\text { FIECTL }\end{array}$ & $\begin{array}{l}107 \\
10 \pi\end{array}$ \\
\hline$? 4$ & r. & 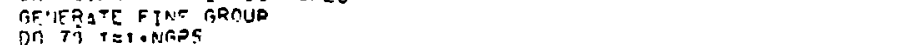 & fTargL & 109 \\
\hline 75 & & 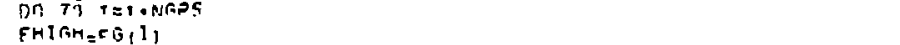 & FTFCOL & 110 \\
\hline 26 & & 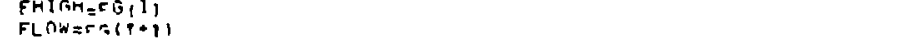 & $F T=C A L$ & $11 !$ \\
\hline 37 & & 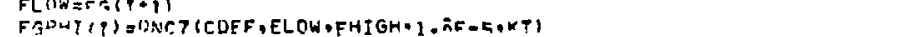 & $\begin{array}{l}\text { FTACOL } \\
\text { FTACOL }\end{array}$ & 112 \\
\hline$=\mu$ & 70 & CONTIUlE & GTACOL & 114 \\
\hline & & (sก) Ti & FTirOL & 115 \\
\hline $3 n$ & 80 & COSTITHE & ETACAL & 116 \\
\hline$\because$ & & WDITE $(I 116 \cdot 1 \mathrm{nn})$ & FTAROL & 117 \\
\hline 3? & & 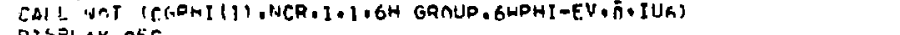 & FTHCOL & $11 \mathrm{H}$ \\
\hline 33 & r & 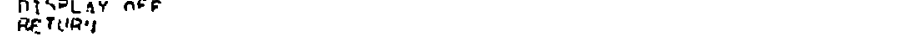 & $\begin{array}{l}\text { FTPCOL } \\
\text { FTarnL }\end{array}$ & 120 \\
\hline 36 & $\bar{c}$ & FONMAT in & $\begin{array}{l}\text { FTarnL } \\
\text { FTarnL }\end{array}$ & \{? \\
\hline $3 \mathrm{~h}$ & 109 & FDUMAT (ACOAASE GROUF FLUX VALUES ISEN IN COLLAPSFA) & FTRCAL & 123 \\
\hline & & ENO & FTHCOL & 128 \\
\hline
\end{tabular}


InInEX $n ? / 19,75$

SURROUTINF FLUX

2 THEE $Y$ HEFFHFNCER VARIAULES

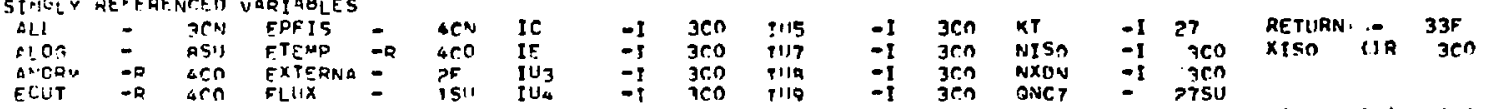

LLIIX

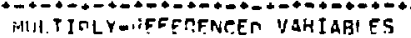

MIIT.TIRLYAIFFESFNCER VAHIABI ES

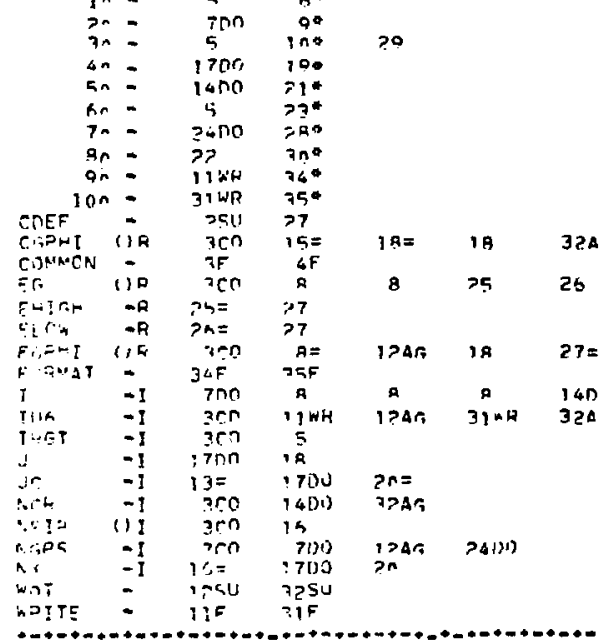

$\begin{array}{lllllllllll}15 & 15 & \text { in } & 19 & 2400 & 25 & 36 & 27\end{array}$

INREX CP/?R/75

FUMICTION COEF (EX)

\begin{tabular}{|c|c|c|}
\hline 1 & & 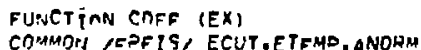 \\
\hline$?$ & & COPMOH KEPFIS, ECUT,ETFMD, ANOHM \\
\hline$?$ & & IF (EX.GE.ECUT) GO TO IS \\
\hline 4 & & CDFF $=\triangle A, O D M / F X$ \\
\hline $\begin{array}{l}5 \\
6\end{array}$ & & RET(1RA) \\
\hline $\begin{array}{l}5 \\
7\end{array}$ & 12 & CNFF $=S A R T\{E X\}$ EXP $\{-E X / E T F M P\}$ \\
\hline 7 & & RETUAN \\
\hline $\mathbf{q}$ & & ENI) \\
\hline
\end{tabular}

$\begin{array}{ll}\text { FTACAL } & 125 \\ \text { FTRCAL } & 126 \\ \text { FTOCAL } & 177 \\ \text { FTACELL } & 128 \\ \text { FTFCOL } & 1>9 \\ \text { FTAEAL } & 130 \\ \text { FTIRAL } & 131 \\ \text { FTACDL } & 132\end{array}$

INREX $\quad 2 / 19 / 75$

FUNCTIINN COEF (EX)

SINGLY REFEQENCED WARIAHLES

CONMAM - PF EPFIS - $2 C^{N}$ EXP - $6 S U$ SDRT $-6 S 11$

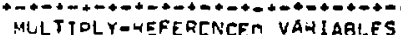

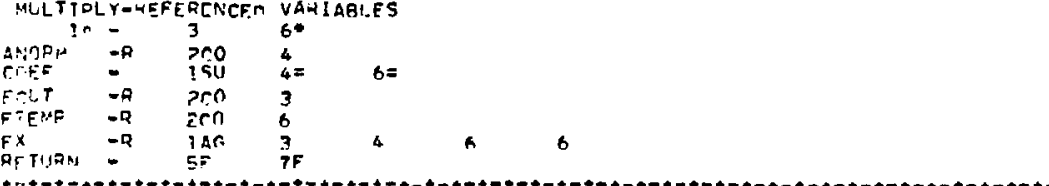




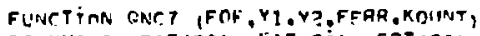

STACOL $\quad 133$

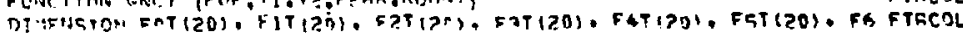

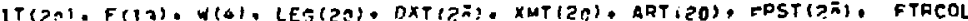

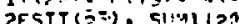

$W(1)=4 i, a_{0}$.

$W(P)=? i 6.194 n$

$H(x)=j 7,144^{N}$.

$W(4)=37$ P. $144 n$.

$A=Y 1$

FPS=FFOR

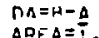

$F S T=1$.

$1=1$

lin $1 \dot{r}, \tau=i, 1 \mathrm{x}$, ?

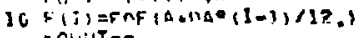

$n \cap_{i+i}+T=7$

20 lix $=n A /=0$

$x, y=A+n x$

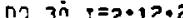

$30 F(1)=F \cap F(A+1, a *(I-1) / 12$.

$h O+A_{i} T=V O I N T \cdot h$

anC $7=:$

IF (KOIINT.RT.50OC) RETUJRN

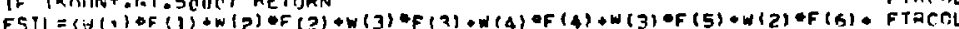

Iw $(1)$ os 7, i or $x$

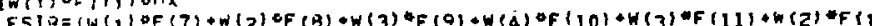

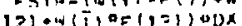

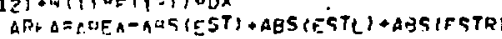

$S 11 N=F C T L+F C T A$

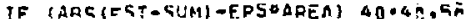

4. IF $(F T T-1.17,7.50 .70$

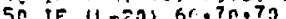

$60 L=+1$

$L \equiv \therefore(L)=2$

F $T(L)=F(7)$

$F_{1} P(1)=,F(a)$

$F>r(6)=F(4)$

FYi (1, I =F(1n)

$F G T(t)=F(1)\}$
$F Q T(L)=F(1)\}$

FG $(L)=F(1, T)$

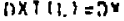

$X M T(L)=X L$

$\triangle D T(L)=\angle O F A$

EDST $(1,=50 S / 1$.

FSPT

$D A=D X$

$F(13)=\sigma(7)$

$F(1)\}=F(4)$

$F(Q)=F(-)$

$F(7)=F(4)$

$F(5)=F(3)$

F(3) $=5(2)$

FST $=$ ESTL

EPS=EnCT (L)

GO TO $\rightarrow 0$

70 if (Lec $(1)-P$ ) $90+8 c=80$

DO 5UM) (L) $=$ elum

LEG(L) $=1$

$A=X M T(1)$

$D A=C X T(L)$

$F(I)=F \cdot T, L)$

$F(3)=F(t)$

$F(4)=F+T+1)$

$F(7)=F+T$ (I)

$F(9)=r 4 T$ ( )

F 111$\}=F 5+$ IL)

$F(17)=56 \mathrm{~T}(\mathrm{~L})$

$\triangle A F A=C D T$ R I

EST $=E S T T$ II.

EFC=ECatil

(io $\mathrm{T} n=0$

90 SU:A=Siml $(1)+$ SUM

$L=L-1$

I5 (L-7) $10 n \cdot 100 \cdot 70$

100 OHCT:S11

RETLIRI)

ENO 


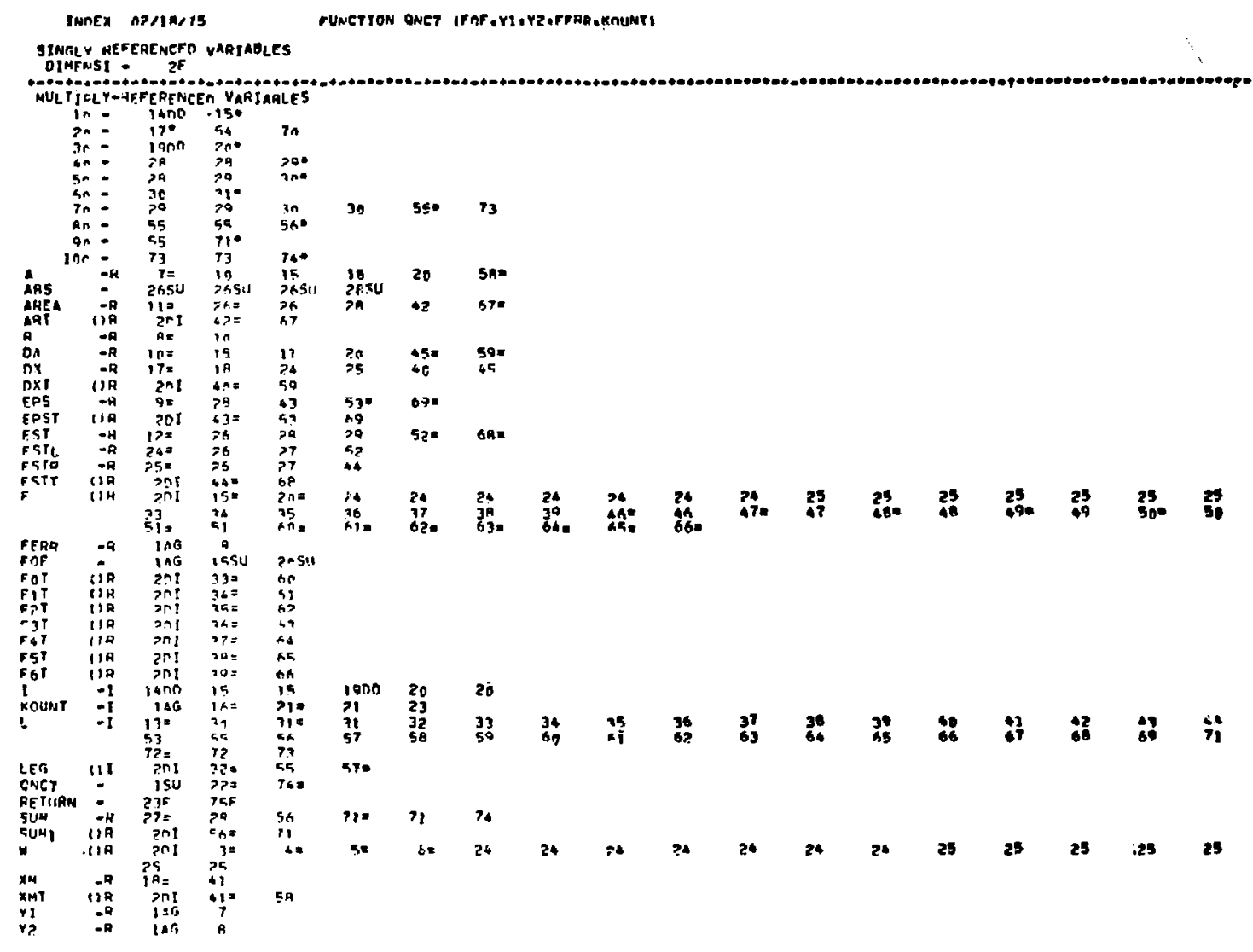

IN.EX CZ:T9/75

SHAHOUTINE SENCW\{RETNR

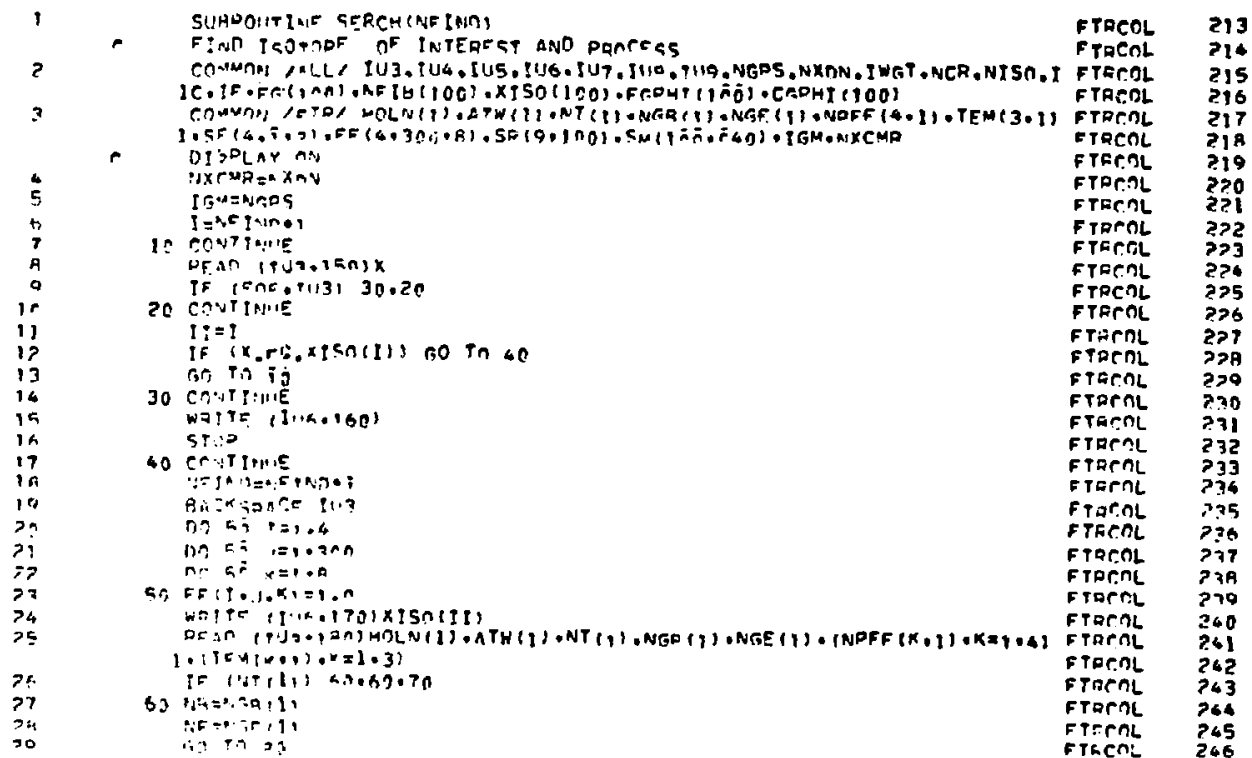




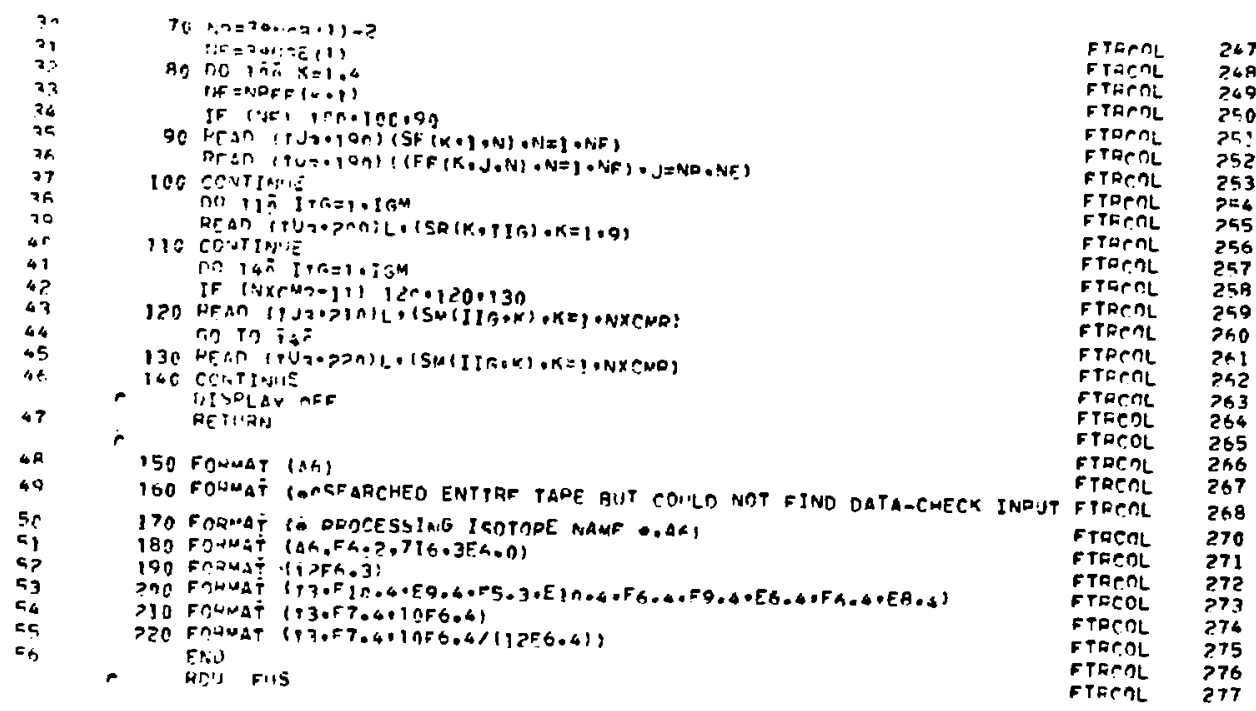

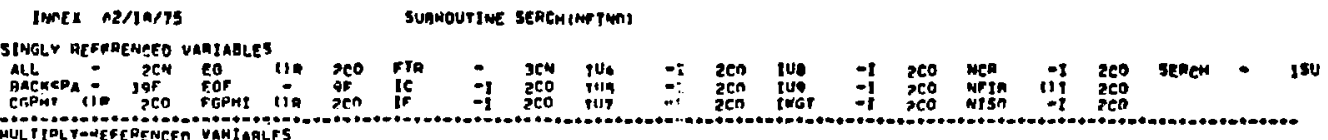

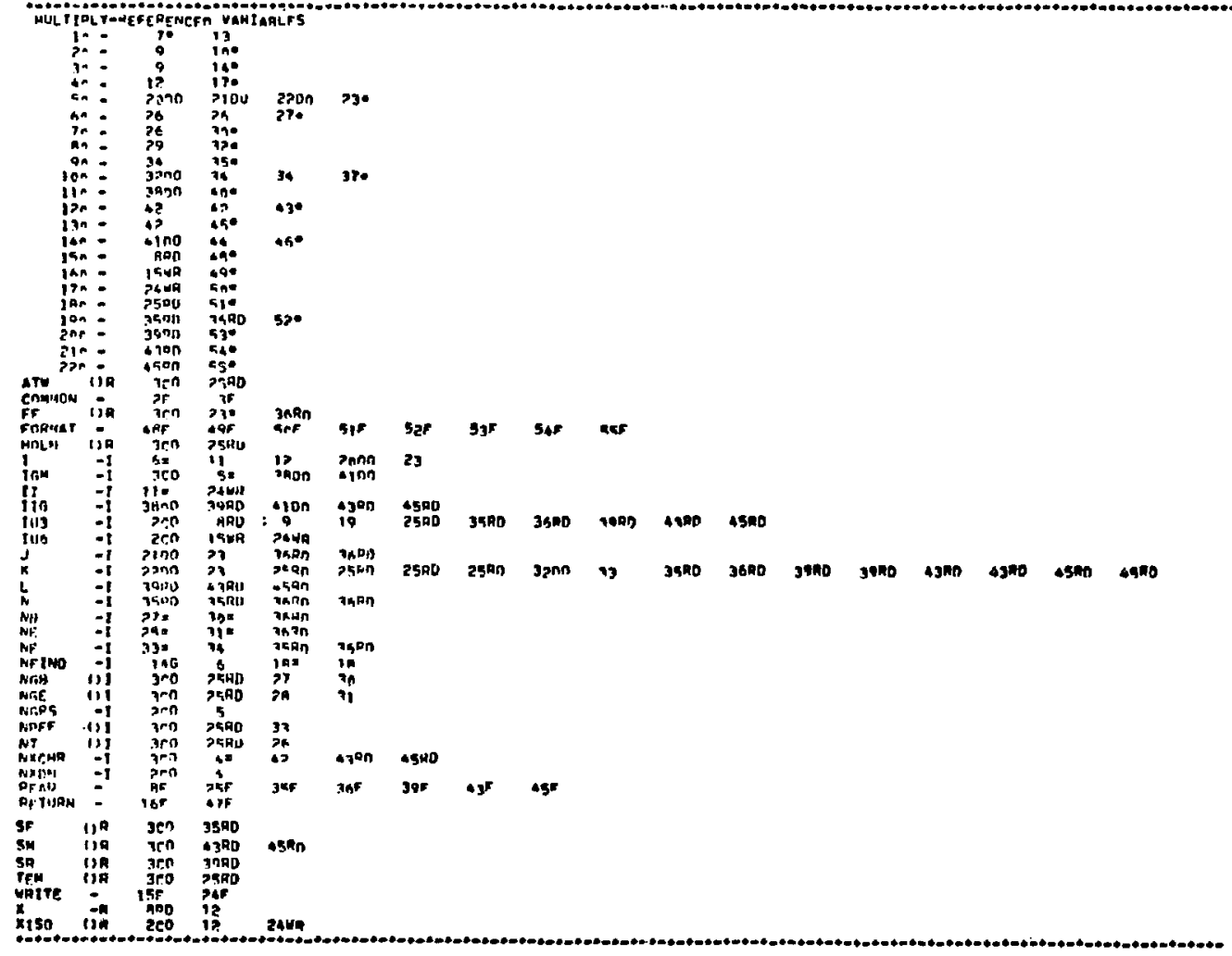




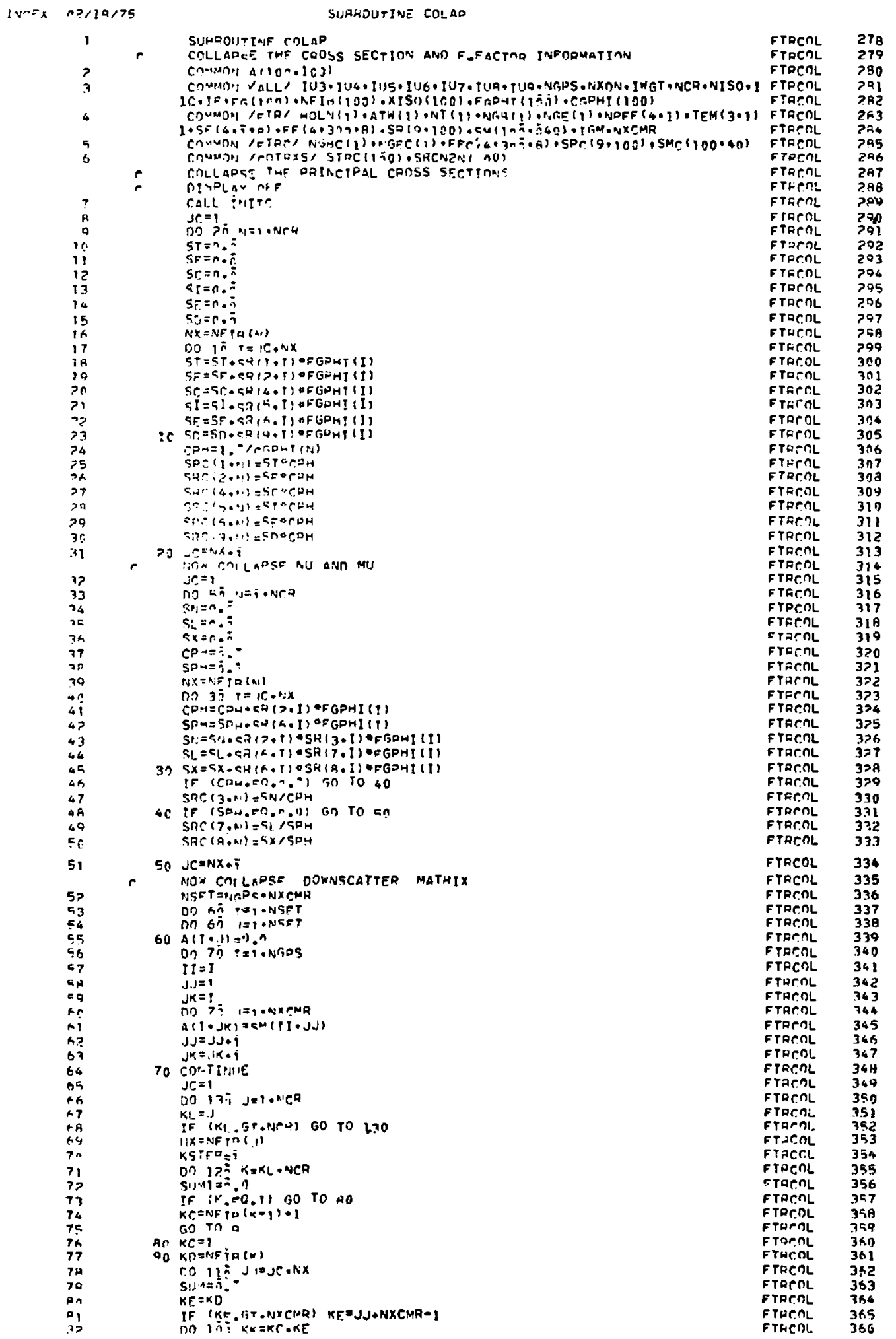




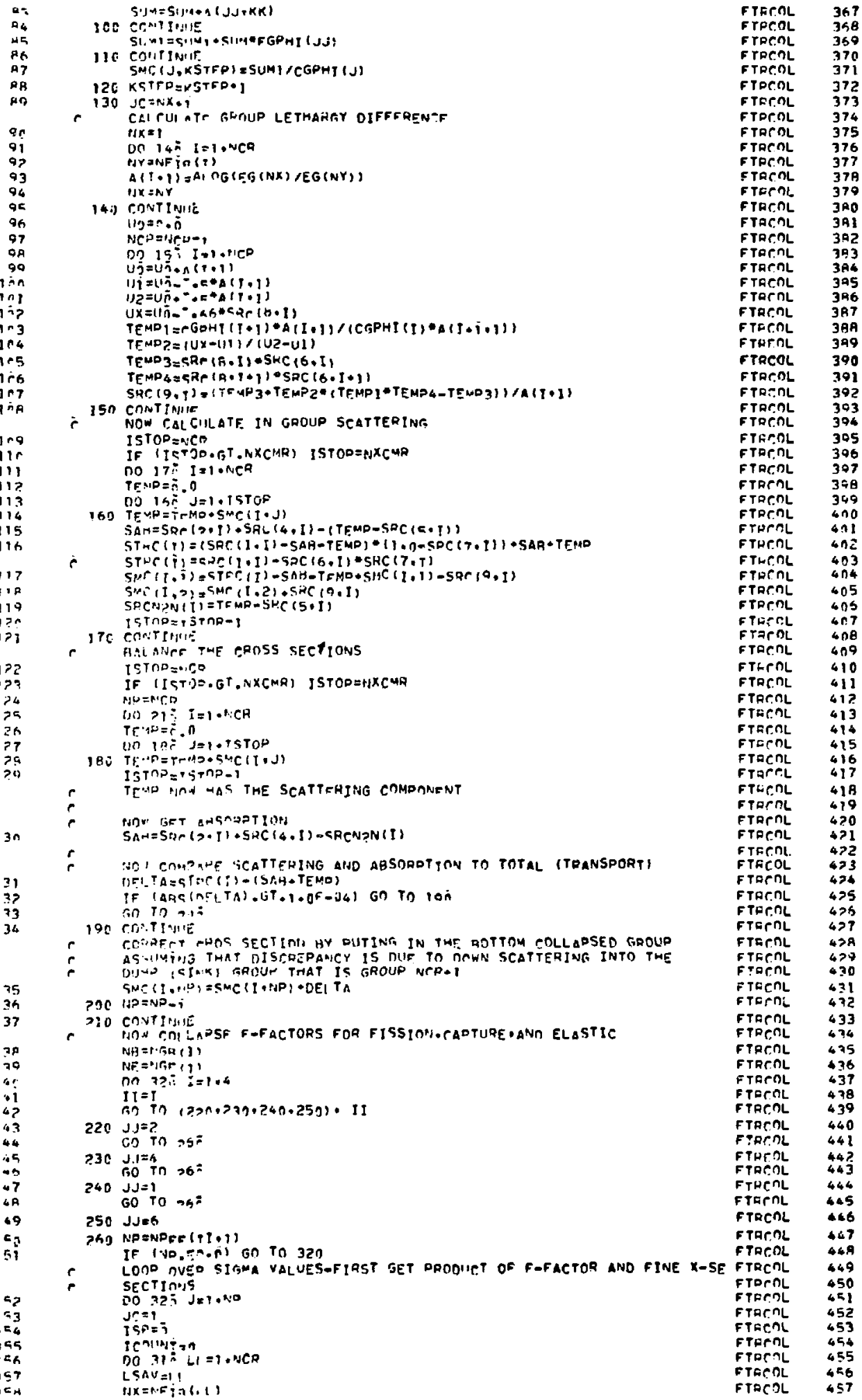




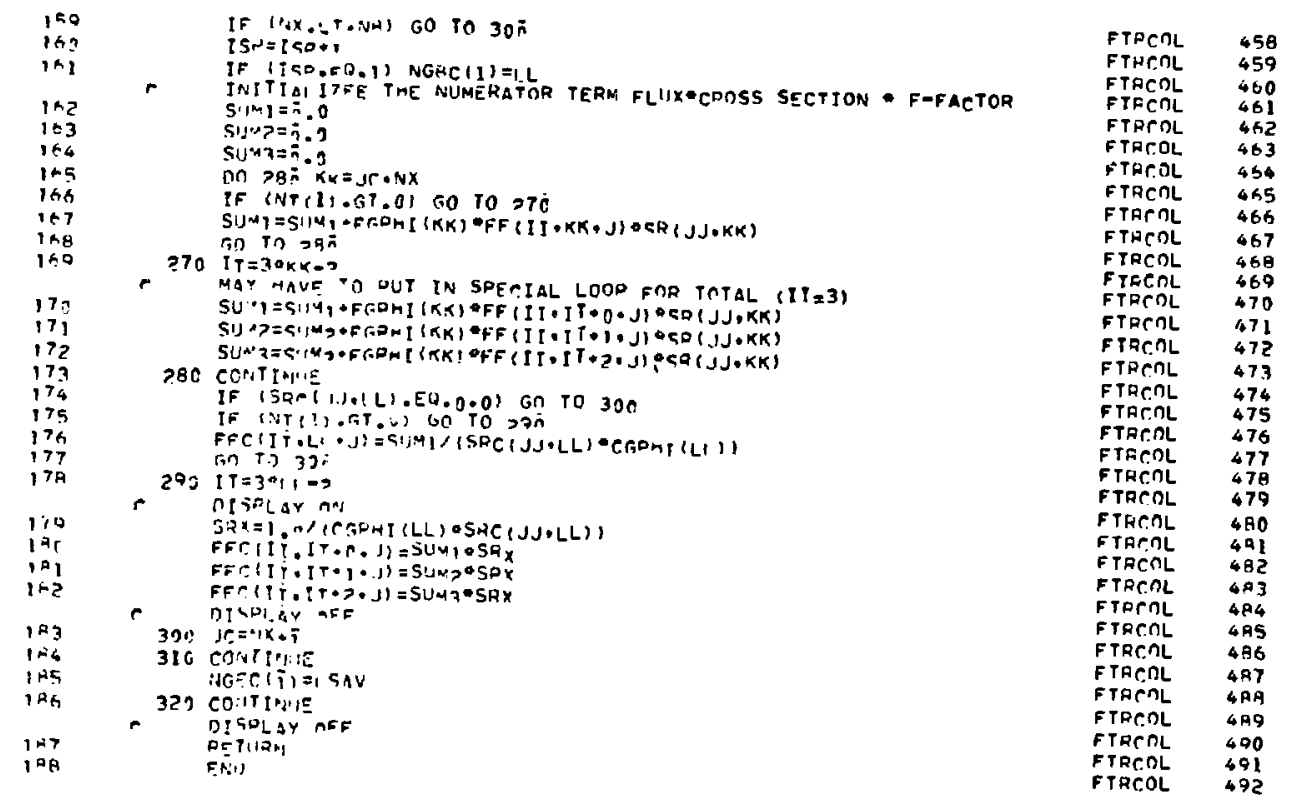

SURAOUTINE COLAP

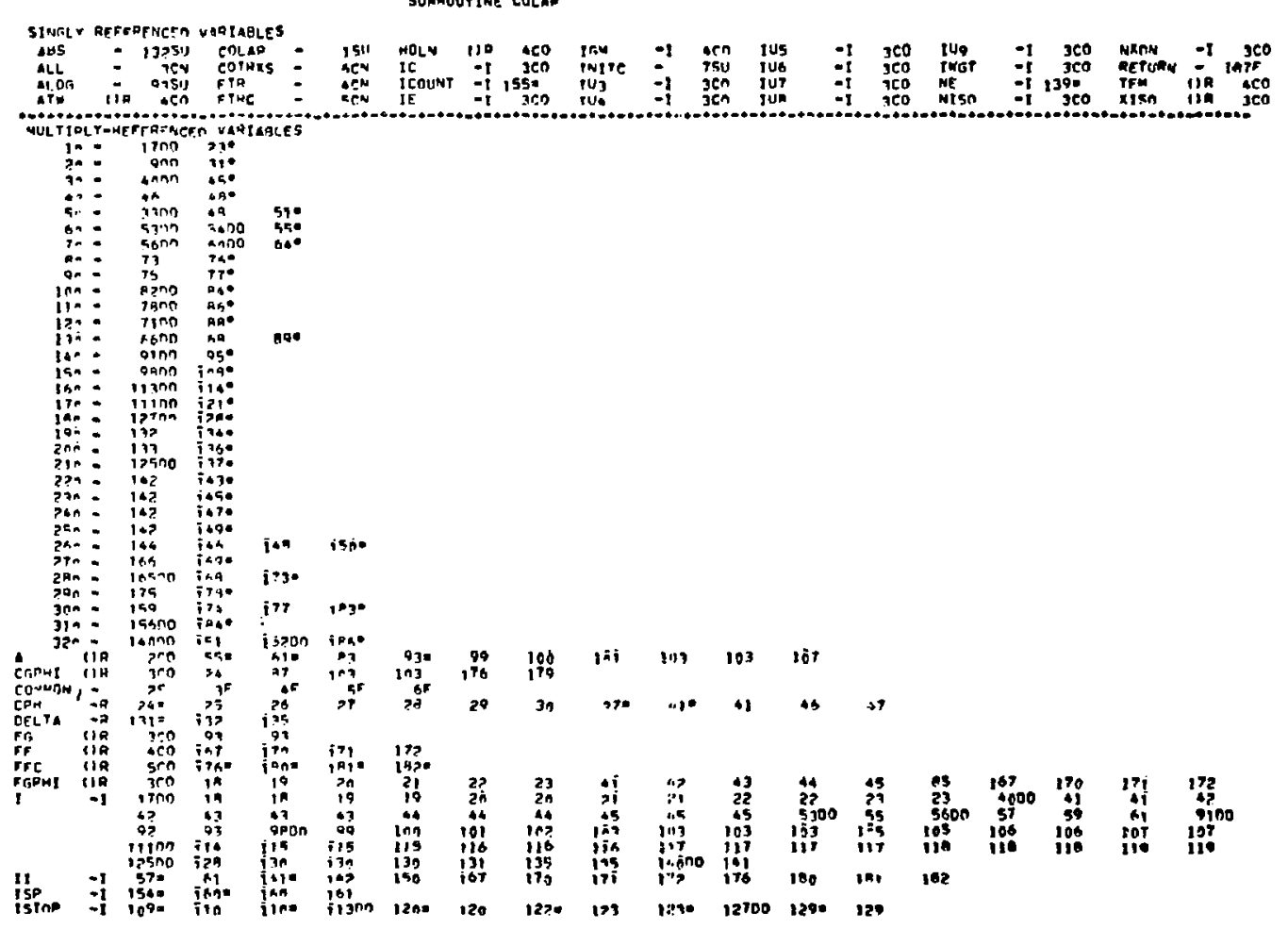



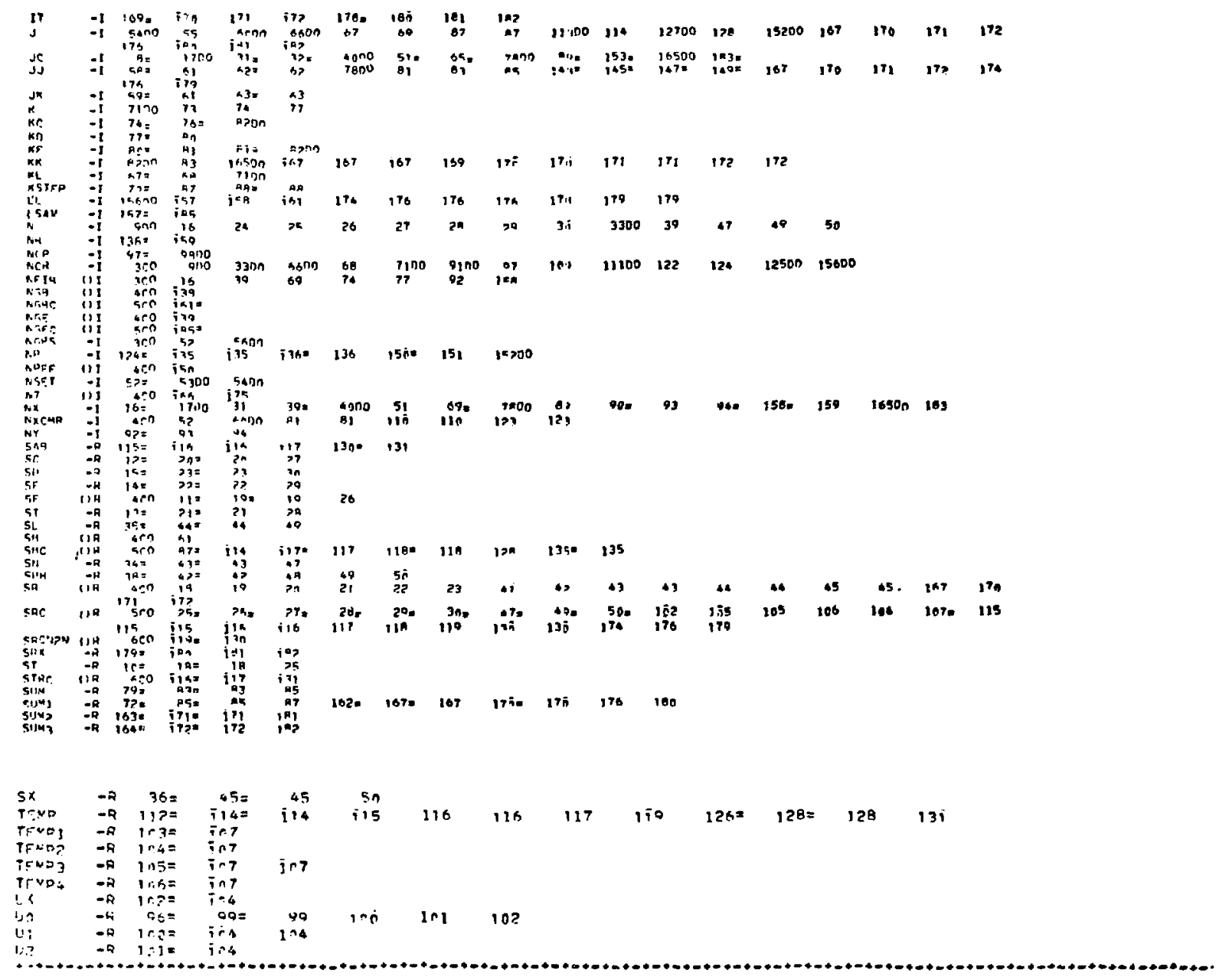

INHFX N?/A/75

SURROUTINE INITC

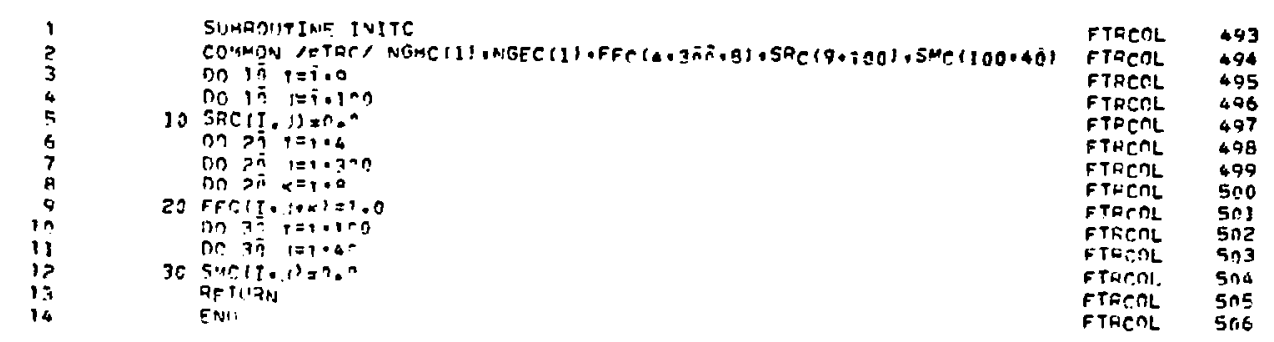


InIfX as,ie/72

SURKOUTINE INITC

SIHEL TEFFRER:CFO UARIABLES

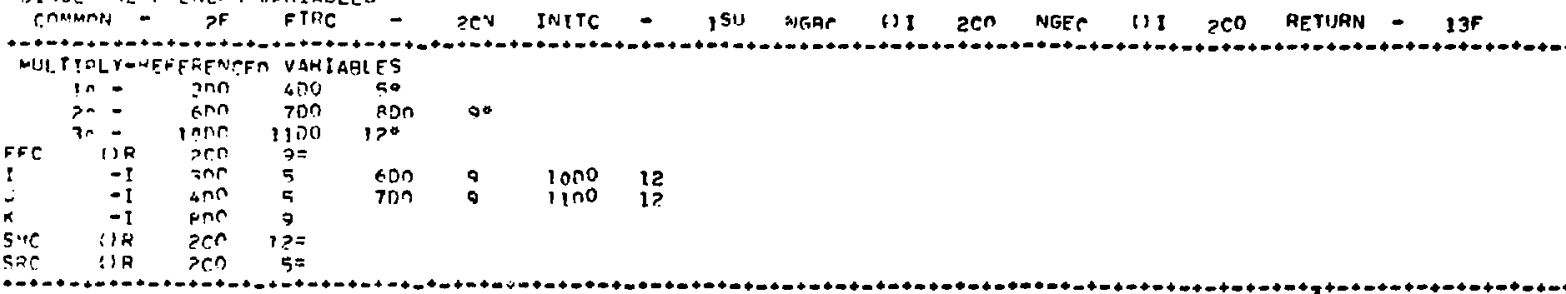

InIfx y?/1日/75

SUAADUTINE RITPUN

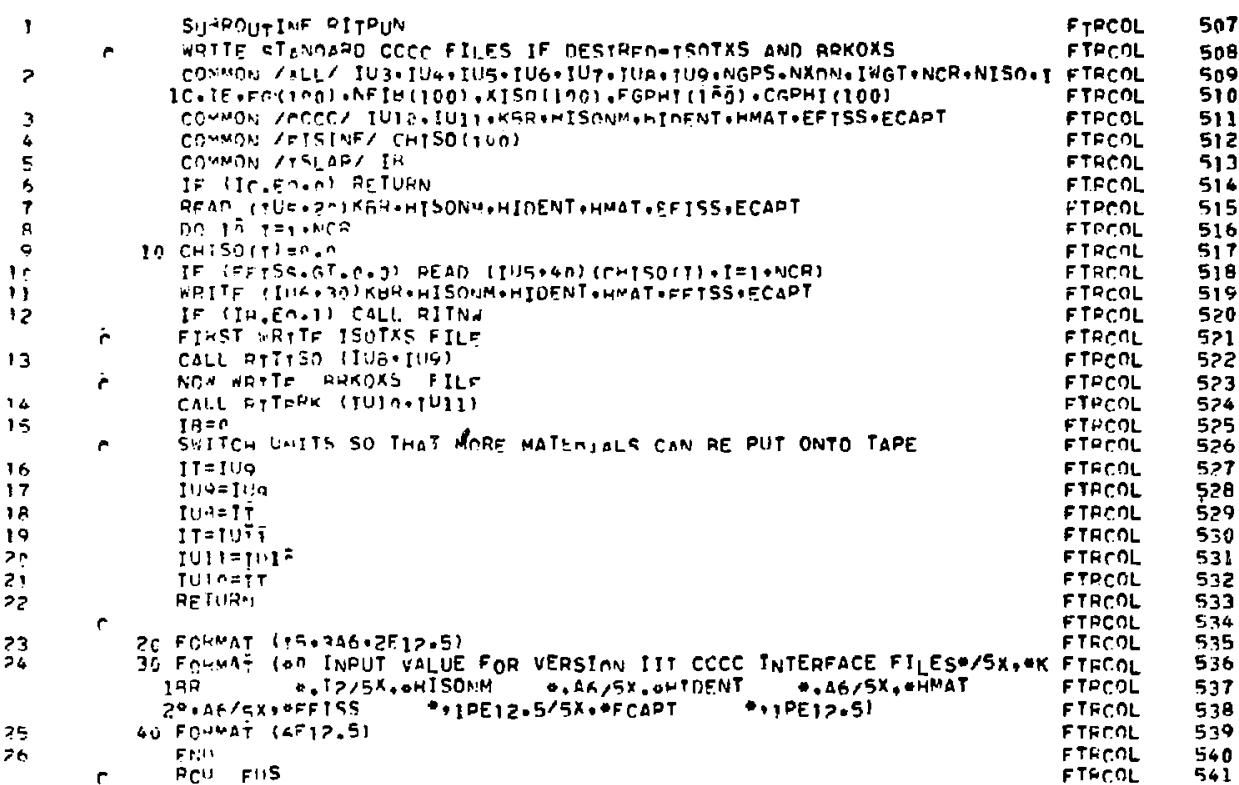

InREX $-5 / 1 R / 75$

SURROUTINE RITPIN

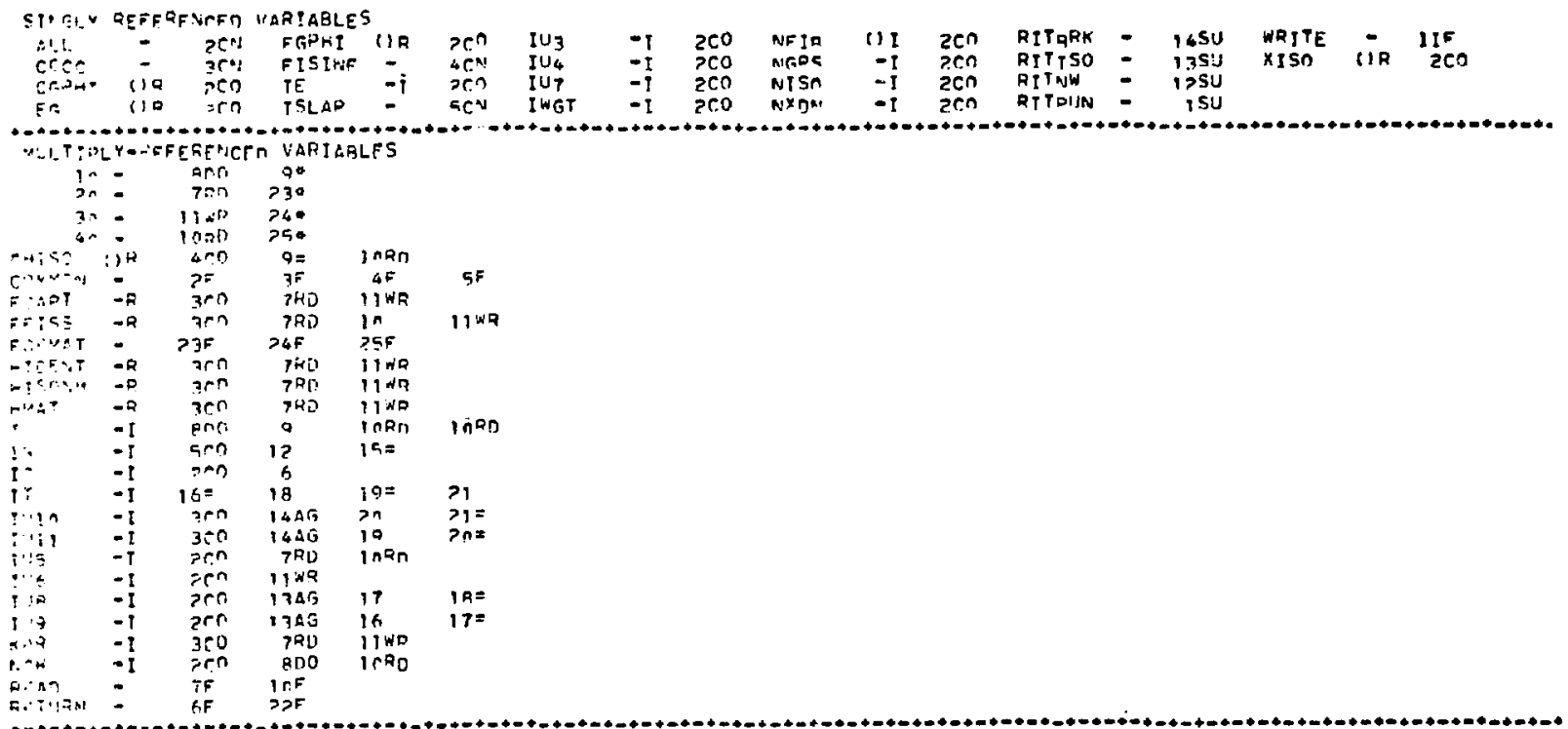




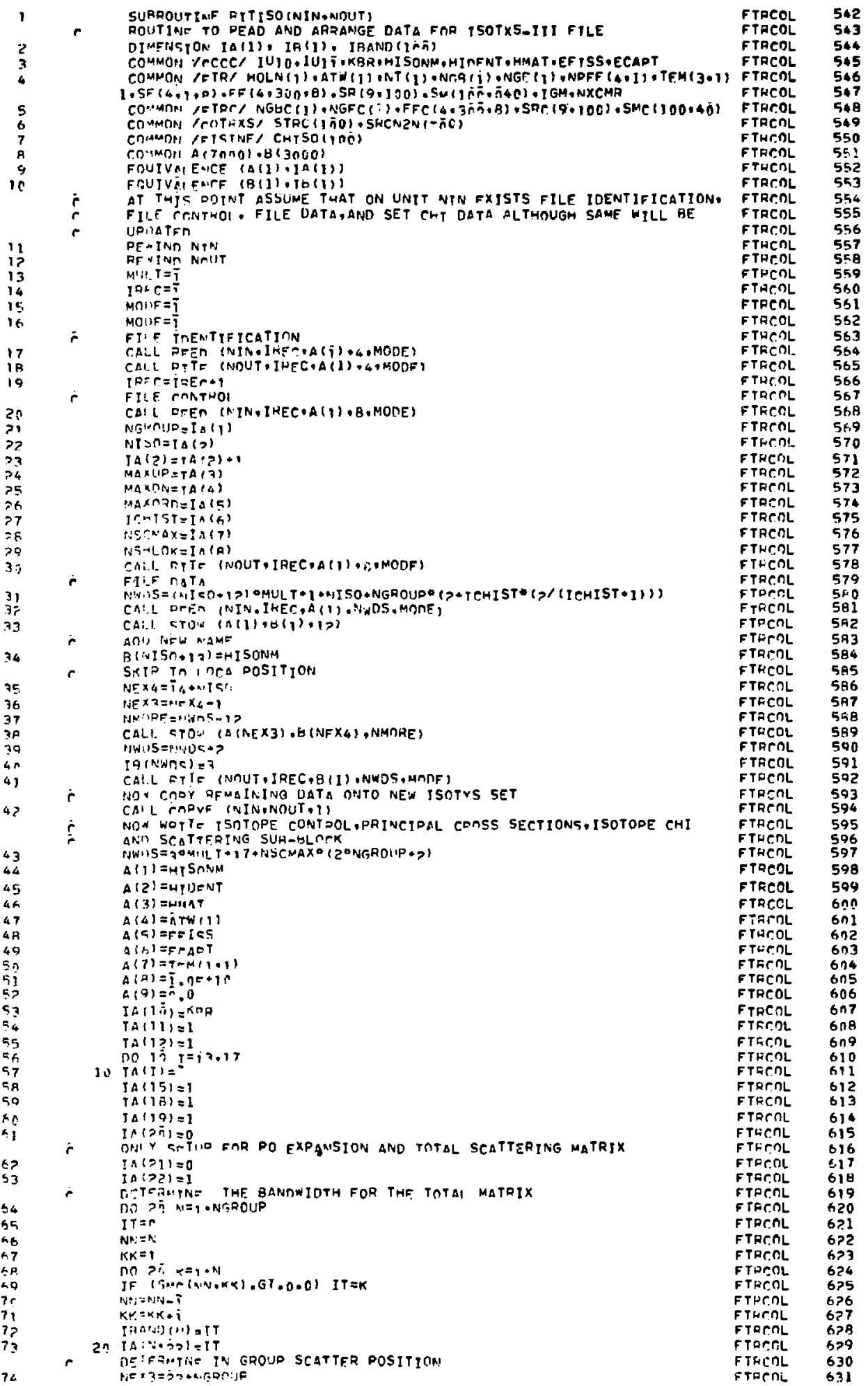




\begin{tabular}{|c|c|c|c|c|}
\hline $7 a$ & & $n$ in $N=1$. NSTLOUP & FTRCOL & 632 \\
\hline 76 & 30 & I $\triangle\left(N E \times{ }^{2}+M\right)=1$ & FTFCOL & 633 \\
\hline 77 & & 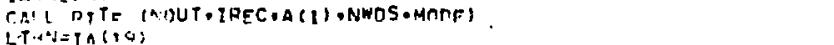 & $\begin{array}{l}\text { FTEENL } \\
\text { FTFEOL }\end{array}$ & $\begin{array}{l}534 \\
635\end{array}$ \\
\hline 79 & $\dot{r}$ & 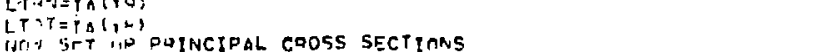 & $\begin{array}{l}\text { FTRCCL } \\
\text { FTPCOL }\end{array}$ & 693 \\
\hline $\mathrm{ac}$ & & 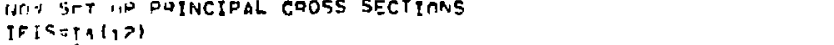 & FTRCOL & $\begin{array}{l}637 \\
6.38\end{array}$ \\
\hline Pi & & BCHI=іs & FTOCOL & 6.39 \\
\hline 87 & $\dot{r}$ & 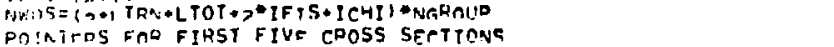 & $\begin{array}{l}\text { FTHCOL } \\
\text { FTFCOL }\end{array}$ & $\begin{array}{l}640 \\
641\end{array}$ \\
\hline $\begin{array}{l}A-a \\
F i\end{array}$ & & 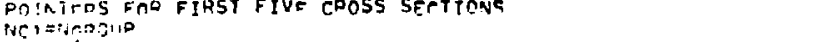 & FTPCOL & $\begin{array}{l}641 \\
642\end{array}$ \\
\hline $\begin{array}{l}F 4 \\
85\end{array}$ & & HC $>=$ SaNGAnUD & FTRCOL & \\
\hline aA & & 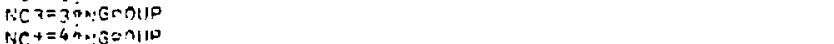 & FTREOL & \\
\hline 9 & & 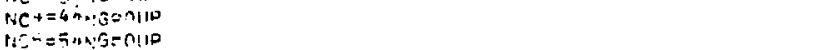 & FTHCOL & 645 \\
\hline क $\mathbf{P}$ & & 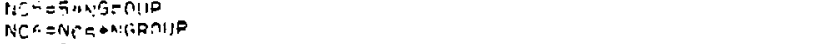 & $\begin{array}{l}\text { FTRCNL } \\
\text { FTRENL }\end{array}$ & $\begin{array}{l}546 \\
547\end{array}$ \\
\hline म9 & & 1) $0 . \% \quad I=1$. NGAROUP & FTECOL & 648 \\
\hline cis. & & $A(J)=2$ the $(J)$ & FTRCOL & 609 \\
\hline$q_{1}$ & & $A(1+N C i)=\operatorname{CoC}(1, \mathrm{~J})$ & FTHCOL & \\
\hline 03 & & $\triangle(j+N C, 5)=5 a C(4 . J)$ & FTACOL & 651 \\
\hline 93 & & $\left.A(1)+N()^{2}\right)=\operatorname{APC}(2 \cdot \mathrm{J})$ & FTRERL & 652 \\
\hline 84 & & $A(7+N(4)=\operatorname{SPC}(3 . J)$ & FTACOL & 653 \\
\hline 95 & & 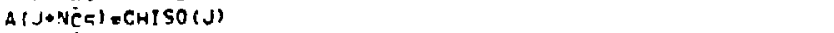 & FTACOL & 654 \\
\hline $9 \mathrm{~h}$ & & 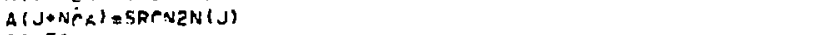 & FTRCOL & 655 \\
\hline 97 & $4 i$ & CONTINHE & FTFCSL & 656 \\
\hline QA & & CALL PTTE \{ADUT IHEC,A(1),NWOS, MBRF & FTRen & \\
\hline & $\dot{r}$ & 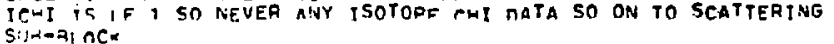 & $\begin{array}{l}\text { FTFCOL } \\
\text { FTRENL }\end{array}$ & $\begin{array}{l}658 \\
659\end{array}$ \\
\hline 49 & & nn $A i n=i$. NGROUP & FTREOL & 669 \\
\hline ine & & NS $=$ I AMND (N) & FTRCOL & 661 \\
\hline ini & & NWOS=E & FTECOL & 662 \\
\hline In? & & $\therefore=P T=$ & FTFCOLL & 663 \\
\hline$i+3$ & & tF (Na.L $=.0)$ S0 To 60 & FTECOL & 664 \\
\hline in 4 & & PHA $=N$ & FTRCOL & \\
\hline ins & & $k K=1$ & FTRCOL & \\
\hline $\ln 6$ & & DO 5 i $k=1$. NA & FTKCOL & 667 \\
\hline $1+7$ & & $N E \times T=P-X T+1$ & FTFCOL & 668 \\
\hline I R & & 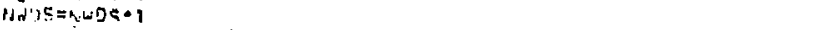 & FTFCOL & 669 \\
\hline ino & & $A(U E X T)=C N C(N N, K M)$ & FTPENL & 670 \\
\hline 118 & & $p \mu y=v i t-i$ & FTaCnL & 671 \\
\hline 11 & 50 & KK=KK, it & FTacol & 672 \\
\hline 117 & & CALL DITE (NOUT,IREC, $L(I)$, NWDS.MADF) & $\begin{array}{l}\text { FTRCOL } \\
\text { FTRCOL }\end{array}$ & $\begin{array}{l}573 \\
674\end{array}$ \\
\hline 113 & 60 & $\begin{array}{l}\text { COATIP, IE } \\
\text { PETUARS }\end{array}$ & FTFEOL & $\begin{array}{l}674 \\
675\end{array}$ \\
\hline 115 & & $\begin{array}{l}\text { PE TuANS } \\
\text { ENH }\end{array}$ & FIFEOLL & 6? \\
\hline & e & $\dot{R C}, \dot{F}: y S$ & FTFEOL & 677 \\
\hline
\end{tabular}

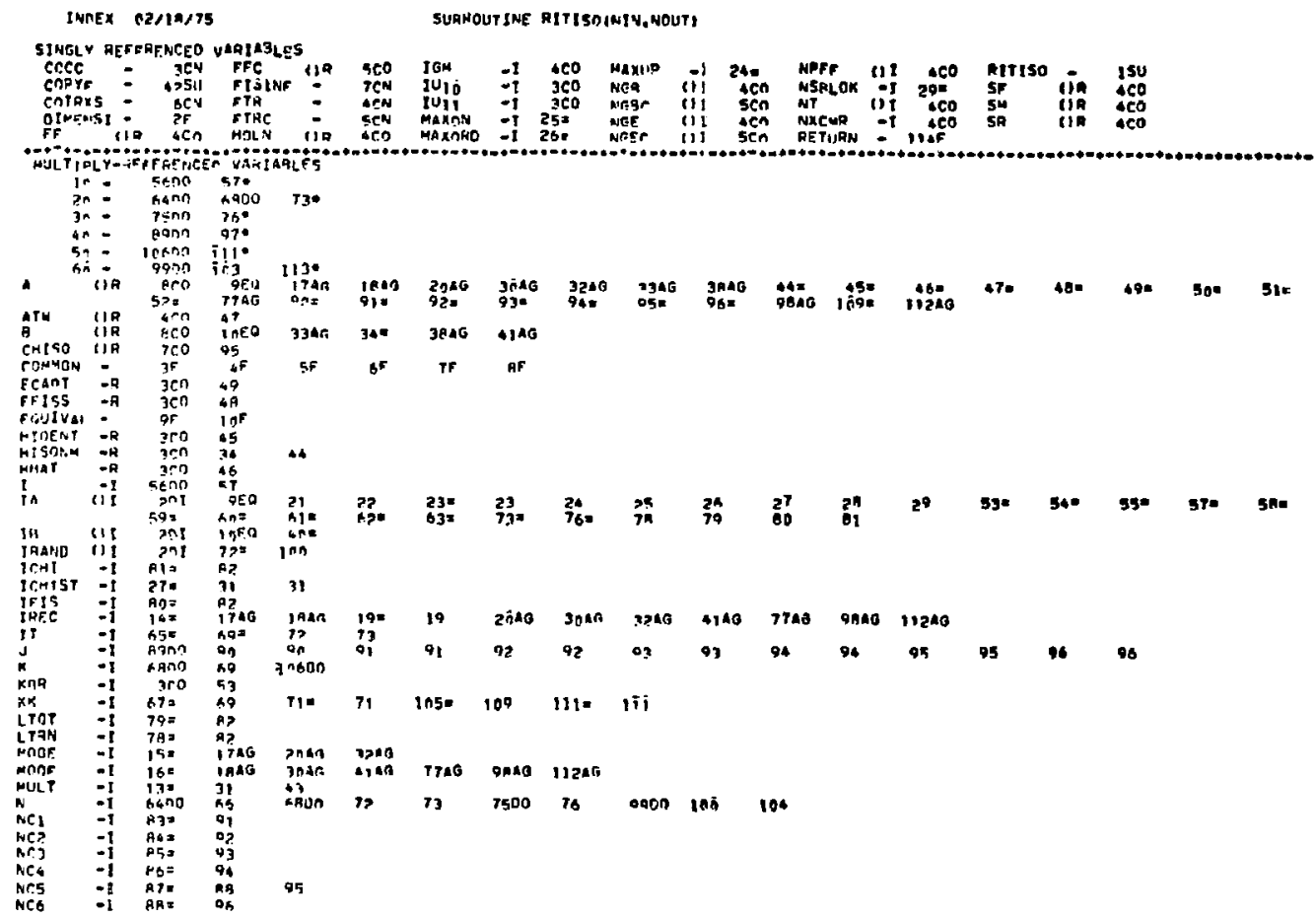




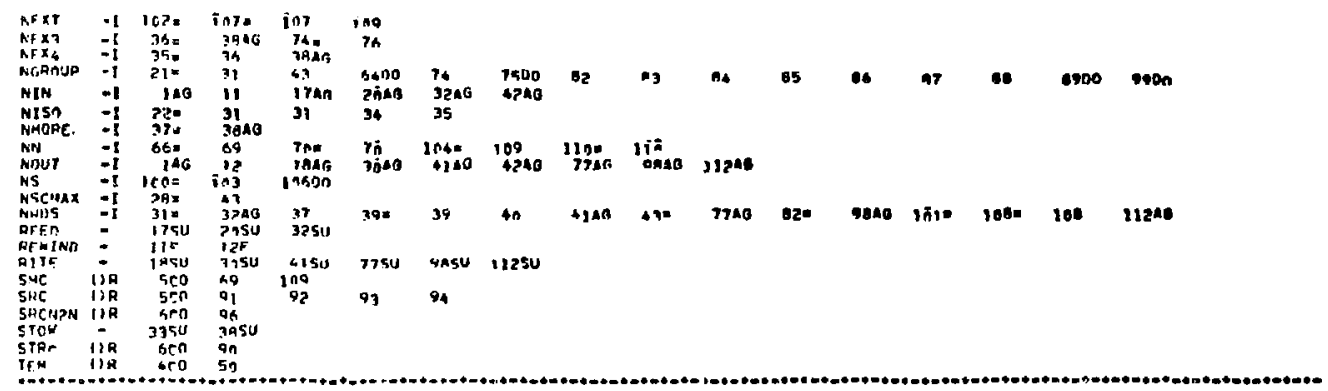

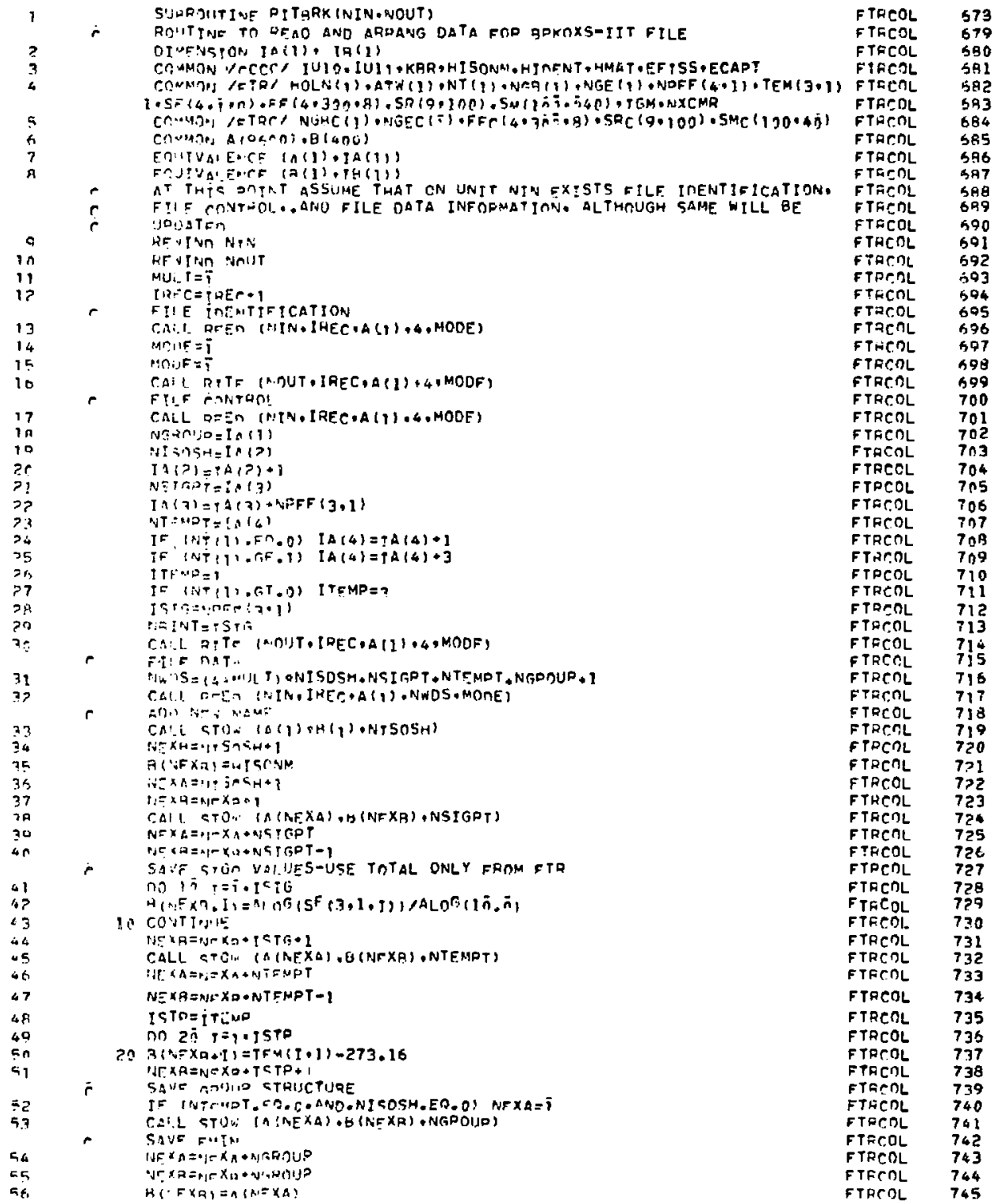




\begin{tabular}{|c|c|c|c|c|}
\hline 57 & & s. $x n_{n}=n_{1}-K_{n}=1$ & FTRCOL & 766 \\
\hline$=8$ & & 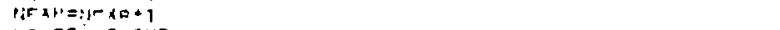 & FTHCOL & 767 \\
\hline & $\mathbf{r}$ & $i, 0 \times 5+5 \pi 01,0$ & FTRent & \\
\hline 59 & & 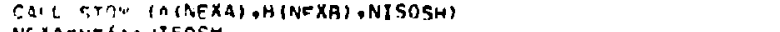 & FTECti. & 7.9 \\
\hline 60 & & Rif $x A=B-x_{A}+N I 5 \cap S H$ & FTACOL & 750 \\
\hline $0 !$ & & 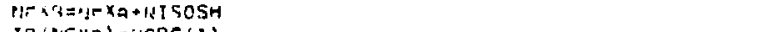 & FTEROL & 791 \\
\hline+2 & & 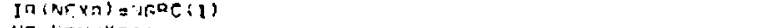 & FTFEOL & 752 \\
\hline$=3$ & & it $\cdot i 4=r:-\times a n$ & FTHEOL & 753 \\
\hline 64 & $r$ & $\begin{array}{l}\text { HI'BHEST RUAIS } \\
\text { CA!L STOH (A (NEXA) . E (NEXA), NISOSHI }\end{array}$ & $\begin{array}{l}\text { FTACOL } \\
\text { Fracol }\end{array}$ & $\begin{array}{l}754 \\
755\end{array}$ \\
\hline $6 \overline{5}$ & & $\therefore A^{-} \times A=A-X \cdots N T S U S H$ & FTACOL & 756 \\
\hline AE & & IJFAB = MI XOQRIT SOSA & FTARAL & 757 \\
\hline 47 & & If $(\operatorname{tex} x)=\mathrm{x}(\mathrm{a})(1)$ & FTHCOL & $748 *$ \\
\hline ar & & 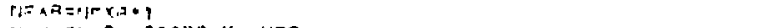 & FTACOL & 759, \\
\hline No & $\dot{r}$ & 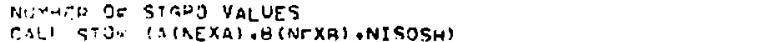 & $\begin{array}{l}\text { FTACOL } \\
\text { FTFrOL }\end{array}$ & \\
\hline $7 r$ & & 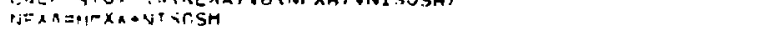 & FTAROL & 762 \\
\hline $7 i$ & & $\operatorname{Hit} \times 4=-12 \times c+2180 S H$ & FTACOL & 763 \\
\hline 77 & & {$[\because(A, F \times 7)=19 i G$} & FTACOL & 7.4 \\
\hline 7.7 & & $n: F \times n=1, \cdots x=+1$ & FTACOL & 765 \\
\hline & $n$ & HITHER CE T-MUERATIHEE VALUES & FTHCOL & 766 \\
\hline 72 & & 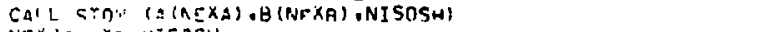 & FTACOL & 767 \\
\hline $7 \div$ & & 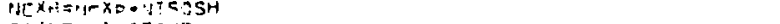 & FTFCOL & 758 \\
\hline $7 \mathrm{~h}$ & & 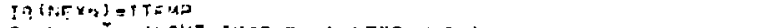 & FTACOL & 769 \\
\hline 77 & & CA: I DTF ('OUT, [UECQH(I) + NEXR MANE) & FTACOL & 770 \\
\hline & $r$ & COLY SRLE-SWIELUING FACINAS ANO CANES SECTIONS & FTACOL & 771 \\
\hline if & & CA:I POPQF (N[NA:ZUITUI) & FraCOL & $77 ?$ \\
\hline 79 & $r$ & $\begin{array}{l}\text { HO ARI AH NEW SFLF SHTELDING FACTORS ANO CROSS SFCTIONS } \\
\text { ISXI:T }\end{array}$ & $\begin{array}{l}\text { FTarOL } \\
\text { Firol }\end{array}$ & $\begin{array}{l}773 \\
774\end{array}$ \\
\hline Pn & & AS: $: F F=N+1 j C(1)-N G 4 C(1)+1$ & FTARAL & 775 \\
\hline $\begin{array}{l}\text { P } 1 \\
\text { P }\end{array}$ & & 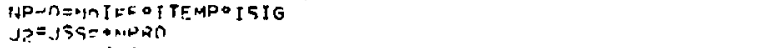 & $\begin{array}{l}\text { FTFEOL } \\
\text { FTOTL }\end{array}$ & $\begin{array}{l}776 \\
777\end{array}$ \\
\hline $3 \frac{3}{4}$ & & 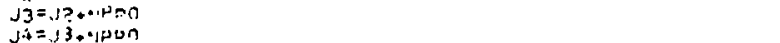 & FTACOL & $\begin{array}{l}779 \\
779\end{array}$ \\
\hline 45 & & 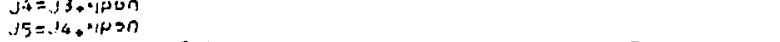 & $\begin{array}{l}\text { FTACOL } \\
\text { FTACOL }\end{array}$ & $7 A 0$ \\
\hline $\operatorname{na}$ & & 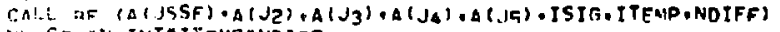 & FTPCOL & $7 B 1$ \\
\hline P 7 & & 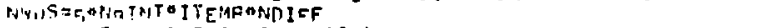 & FTRrOL & 792 \\
\hline a & $c$ & 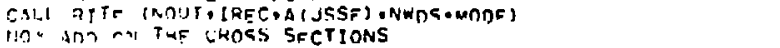 & $\begin{array}{l}\text { FrRent } \\
\text { FTheOL }\end{array}$ & $\begin{array}{l}793 \\
784\end{array}$ \\
\hline $\log ^{n}$ & & 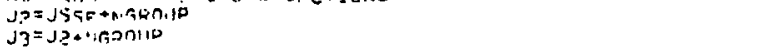 & $\begin{array}{l}\text { FTACOL } \\
\text { FTACOL }\end{array}$ & $\begin{array}{l}7 \text { PS } \\
\text { 7At }\end{array}$ \\
\hline $9 i$ & & $J_{4}=J 3+\cdots G 0$ OIIP & FTAROL & $7 A i$ \\
\hline 47 & & $\sqrt{5}=74 . \cdots 600110$ & FTACOL & 788 \\
\hline 93 & & $J 6=j 5 *$ *GOJIIP & FTACNL & 7ค9 \\
\hline 94 & & CALL XS , A(J5SF),A(J2),A(J3), A(J4), $\triangle(., 15), A(J 6), N$ NROUP) & FTRCOL & 790 \\
\hline 95 & & 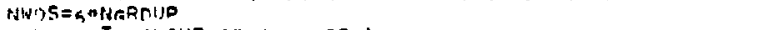 & FTACOL & 791 \\
\hline $\begin{array}{l}96 \\
97 \\
96\end{array}$ & & 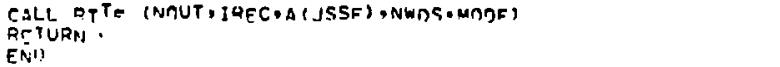 & $\begin{array}{l}\text { FTACOL } \\
\text { FTHCOL } \\
\text { FTACOL }\end{array}$ & $\begin{array}{l}792 \\
793 \\
794\end{array}$ \\
\hline
\end{tabular}

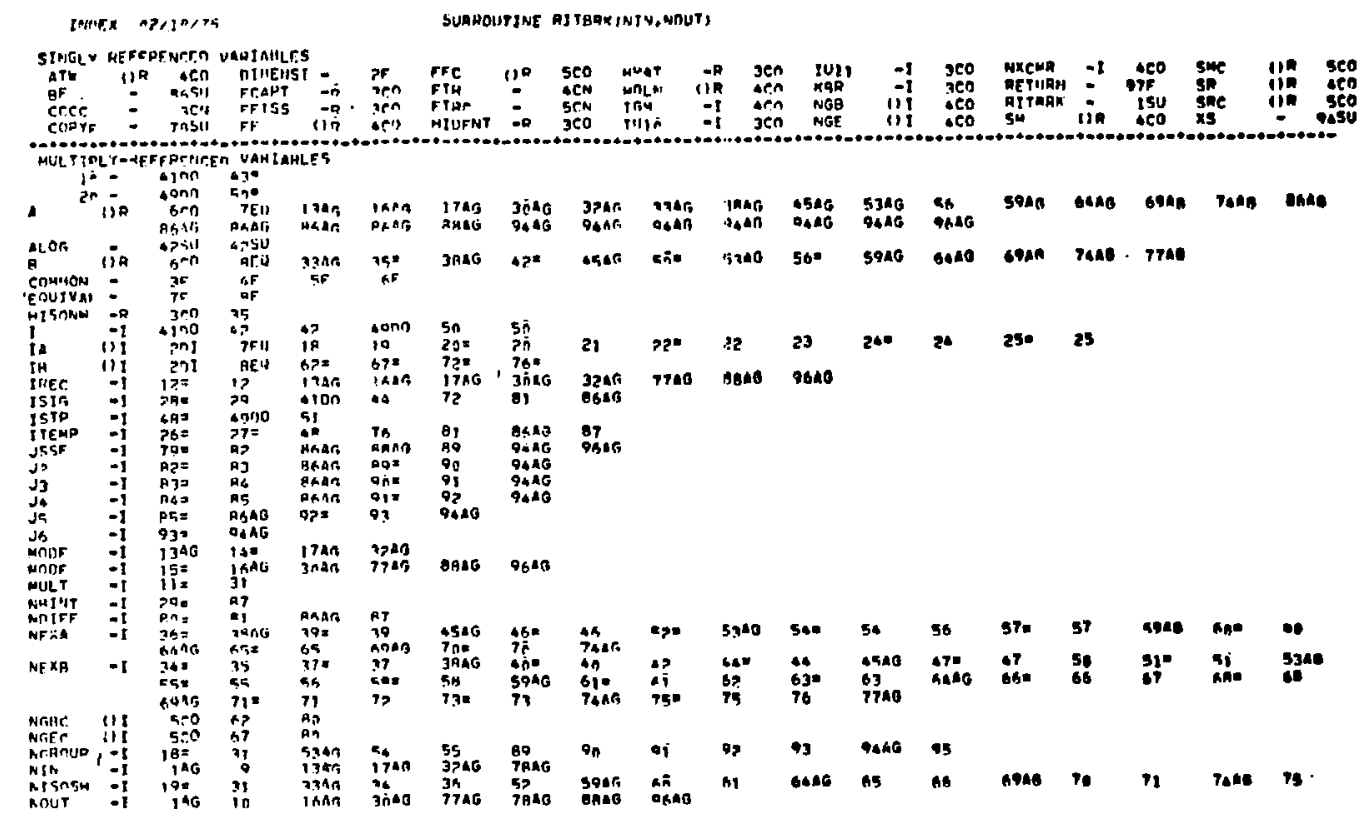




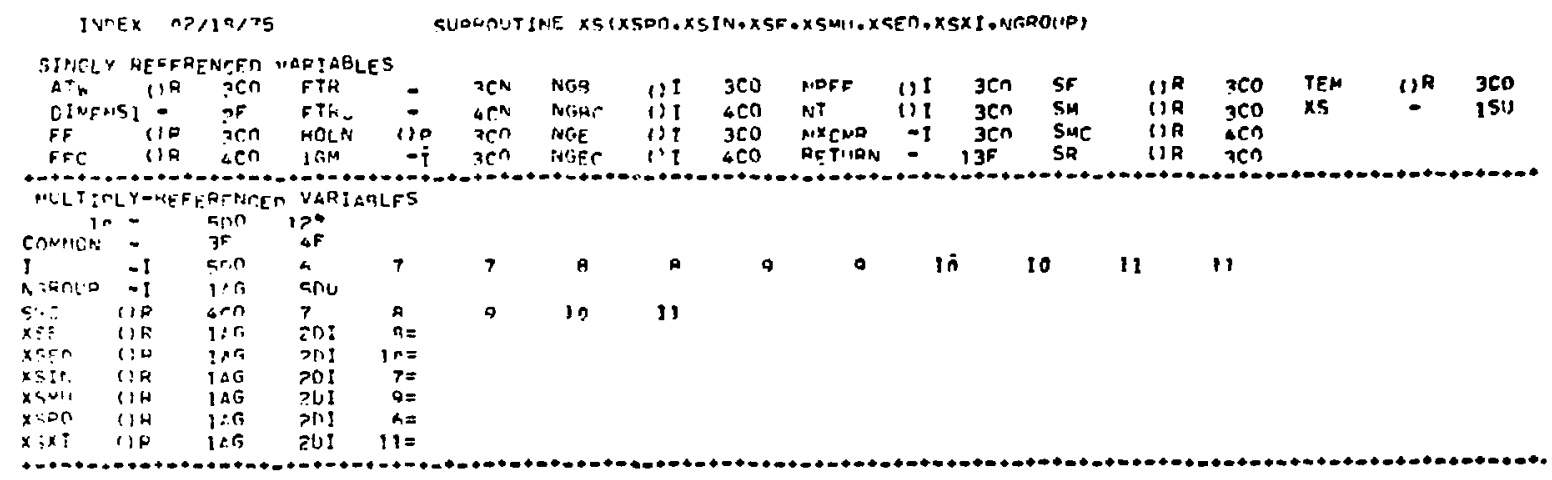

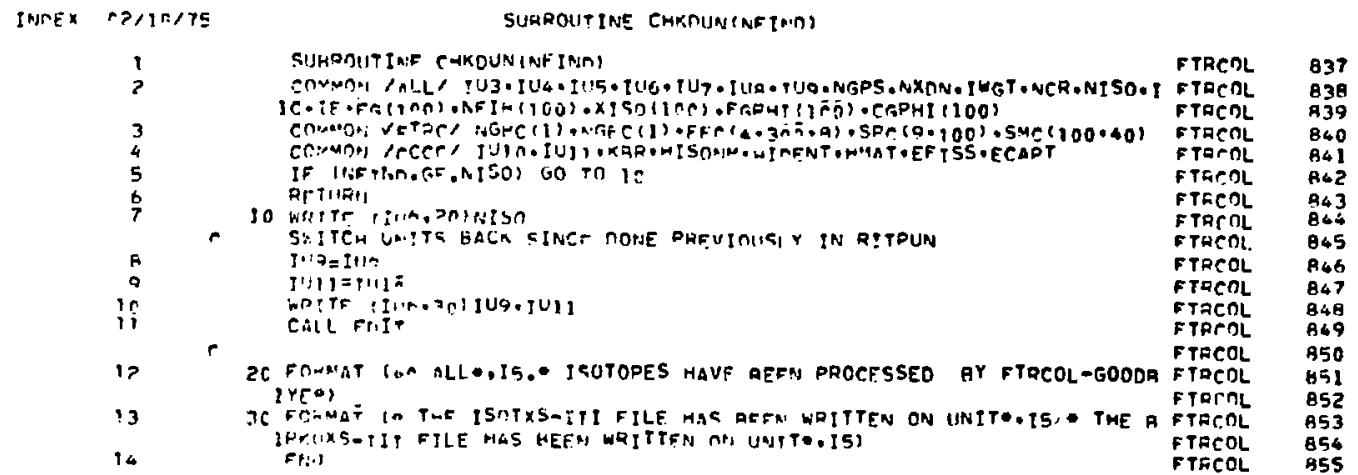

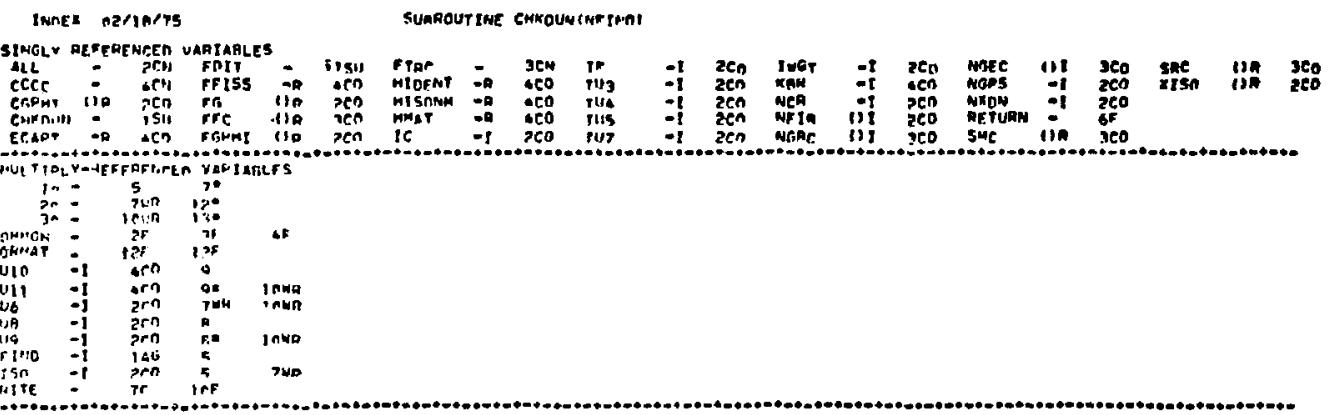



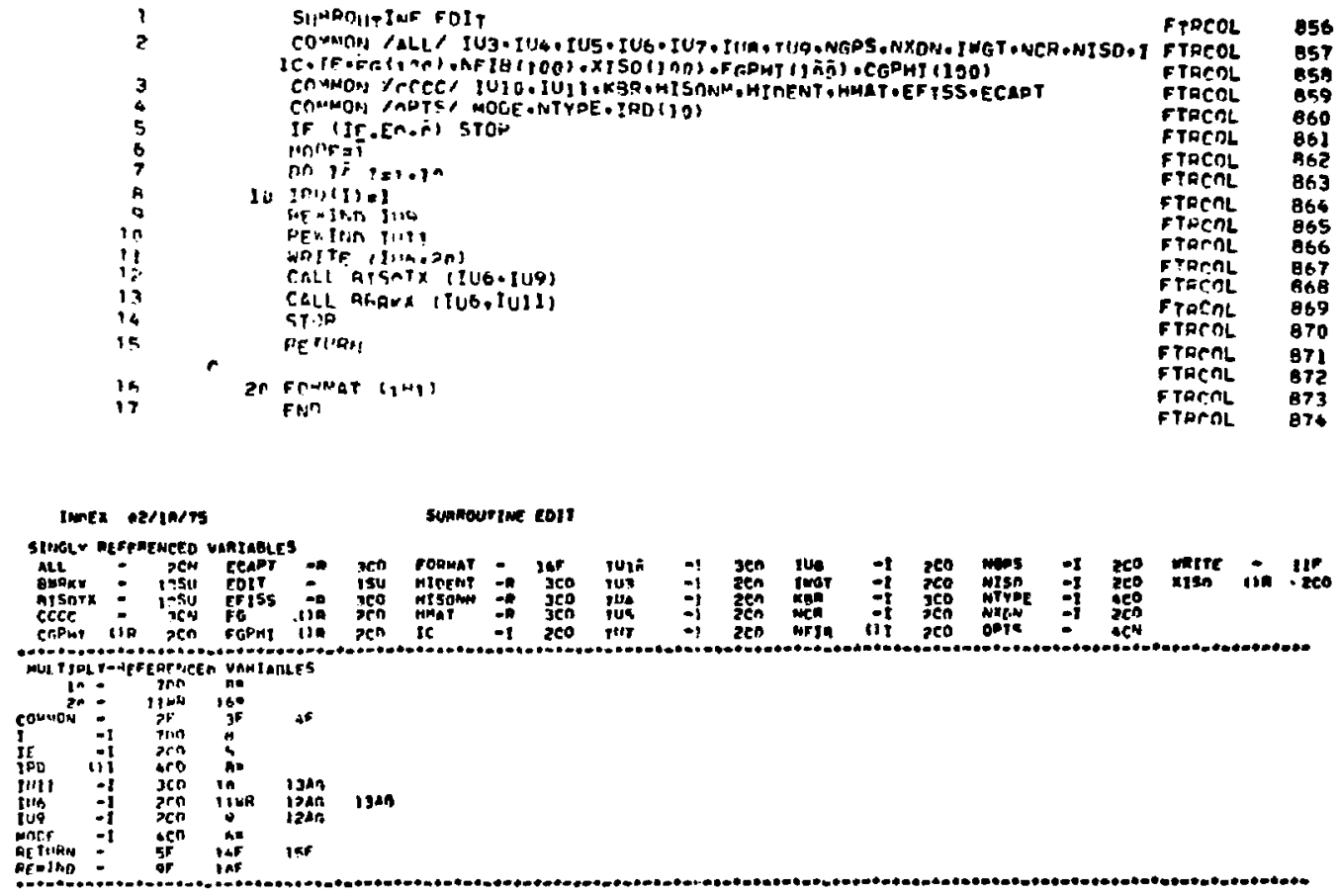

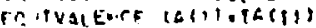

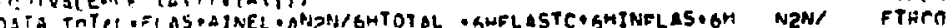

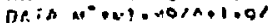

-afila Thentik JCation

$10+r i=i$

inisicis

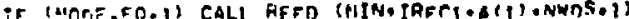

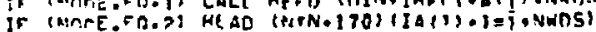

Ft. $=\Delta(i)$

$H i=4+3$

NC: $=171$

Int: $i=; A(B)$

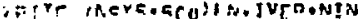

Wn:te persosighmons

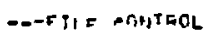

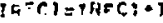

Mudic $=0$

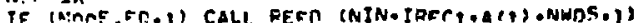

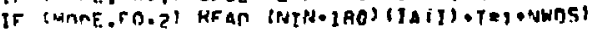

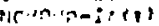

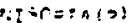

(a)

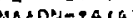

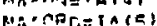

$[C-15 T=J A(6)$

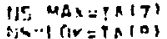


MULT $=\overline{7}$

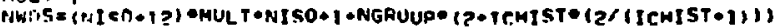

IRFCI IfFECI +1

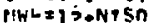

IF (MNAE,FO.1) CALL REFD (NIN.IRFCF.AII) -NWDS.I)

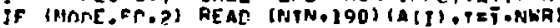

WPITR (NE*5.PPC) (A, I) $1=1 \cdot 12)$

WQTTE $(N E T 5035 P$ )

Un $1 \% T=1.0150$

$J=I+1 j$

IC WhITf. (nSYS- JCh) I. A (J)

THE Txitiontisn

IF IIPHIST.NF. IS 6010 20

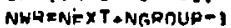

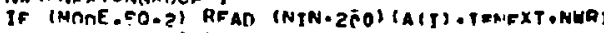

WRITE (RERG $3(n)$

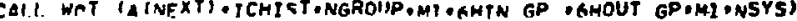

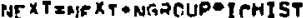

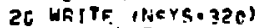

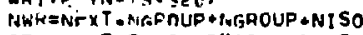

IF (NARE, FA.S) READ (NIN.2CO) (APT), IENFXT ONWA)

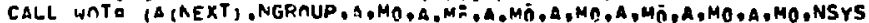
$N W T=P F \times T$

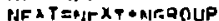

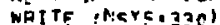

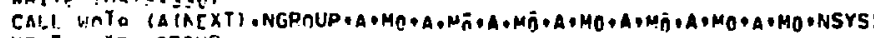

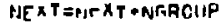

HRITE (NEYS.34UIASNEXT)

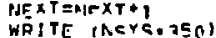

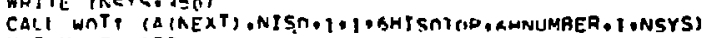

r

If IICHJET,Fn, n) GO TO 40

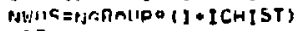

$\operatorname{Hat}=1$

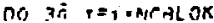

IRECIETPECI+1

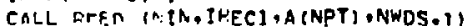

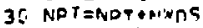

WRITE (MEYS+360)

CALL. WOT (AII) ICHIST,NGROUP,MI IAHIN GD , GHOUT GP,MI ONSYS)

- --ISATCOF CONTFOL AND GROUP INITEPFNDENT UATA

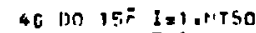

IF (I.T.T. I) WEITE [NSYS 16 $16^{\text {ñ) }}$

HK!

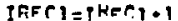

(F (MONE.ED.1) CALL REFD (NIN.IPECI.AII) +NWOS.])

IF (MOAE.FO.Z) REAT (NYN.21O) (AT) T=T NWOS)

JRFAD $=1+R$ WLS

ICHI=TA III)

IFIS=\{ंभ\{|?]

(A) $f=\{A(i)$

IN:H=IA $(1 A)$

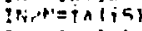

$[P, 1]=! \cap(\lfloor\leq)$

WRITF (ACYS.37nII

WRITE (NEYS.3PC) $(A, I), I=1,6)$

WRTTE. (AEYS.39C) (AII) Y $Y=7,11)$

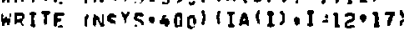

NEXI=⿱一𧰨

LTITIT $=$ is (iA)

LTRAN=TA(19)

ISTRPNEI I $\triangle\left(\mathrm{S}^{A}\right)$

WPITE INGYS-410)LTAT LLTRAN

WRITF (NGYS.420)

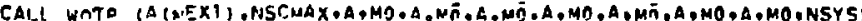

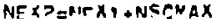

NSTTF $(N<Y<, 430)$

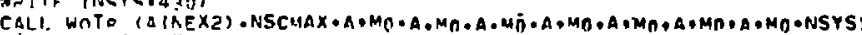
$J i=N E_{-} X \rightarrow \infty, C \operatorname{CBAX}$

WOITE (I:CYS.443)

CAIL WOT, (A) JI) INGKOUP, NSCMAX,MI, GH GDO IGH RLOCK,M) ,NSYSI

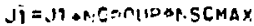

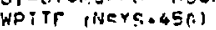

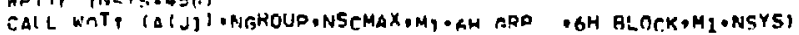

-apRincipal choss SEctions

HHIIS=

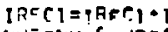

NHW $R=$ NWIS

FTRCOL

FTACOL

FTACOL

FTaCOL

FTRCNL

FTaCOL

FTPCOL

FTRCOL

FTREOLL

FTACOL

FTaCOL

FTRCNL

FTACoL

FTRCOL

FTRCOL

FTRCAL

FTACOL

FTRCDL

FTACOL

FTPCDL

FTRCOL

FTreal

fTreal

FTACOL

FTfEnt

FTPCal

FTRCOL

FTRCOL

FTRCOL

FTFCOL

FTRCOL

FTRCOL

FTREOL

FTarol

FTarol

FTACOL

FTACAL

FTFrol

FTECOL

fTacol

FTHEOL

FTRTOL

FTRCOL

FTHEOL

FTRCOL

FTRCOL

FTACDL

FTPCOL

FTRCOL

FTRCOL

FTRCOL

FTRCOL
FTACOL

FTREOLL

FTPCOL

FTPC?

FTPCOL
FTRCOL

FTRCOL
FTREOL

FTREOL

FTFCOL

FTRCNL

FTRCNL
FTRCAL

FTRrOL

fiarne

FTACOL

FTACOL

FTPCOL

FTACOL

FTRCOL

FTRCOL

FTRCOL

FTRCOL

FTRCOL

FTPCOL

FTACOL

FTaCOL

FTRCDL

FTRCOL

ftaral

FTPCOL

F THEOL

FTFCOL

FTPCOL

FTPCOL

FTFCOL

FTLCo:

FTHCOL

FTRENL

fTRCOL 


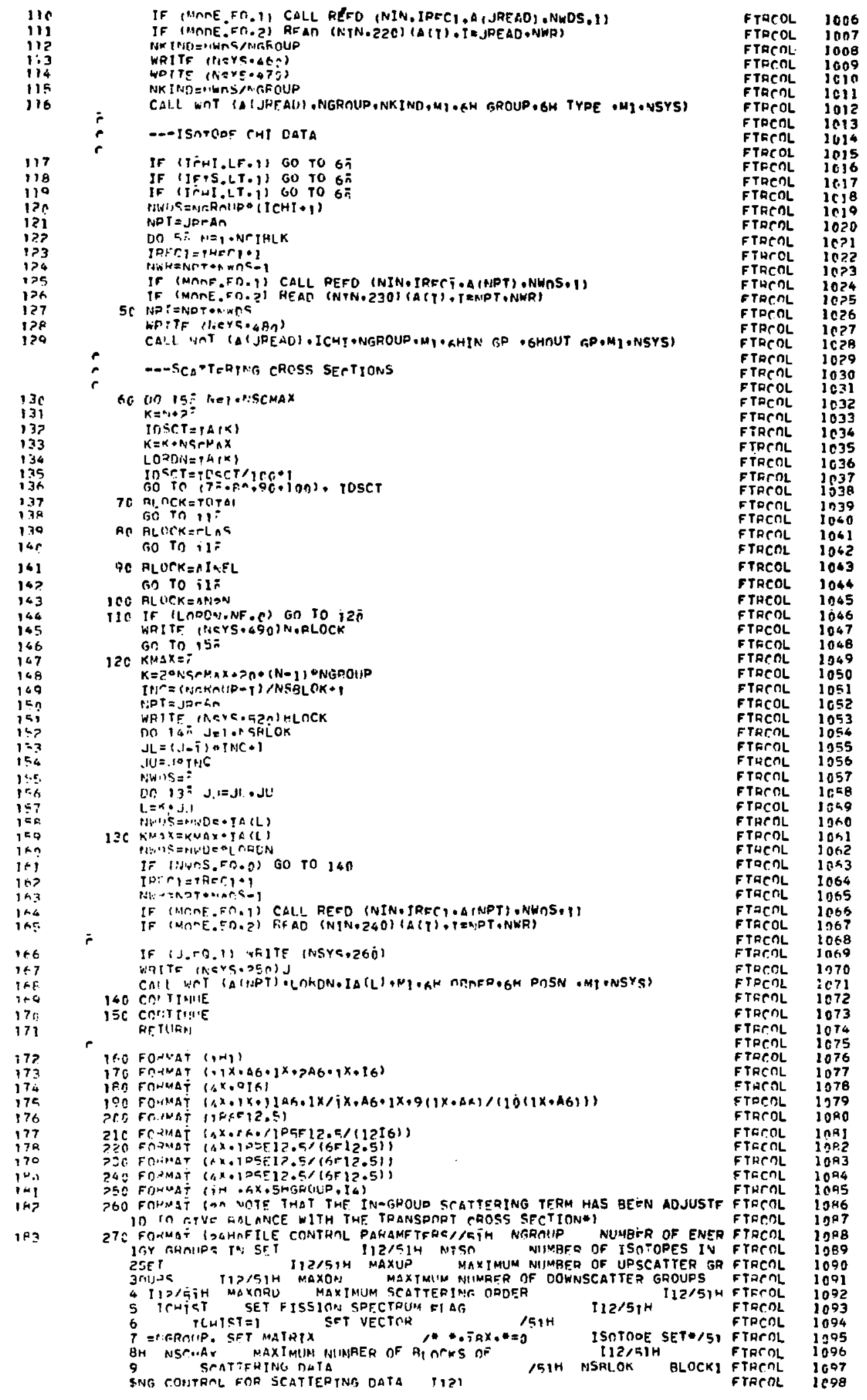




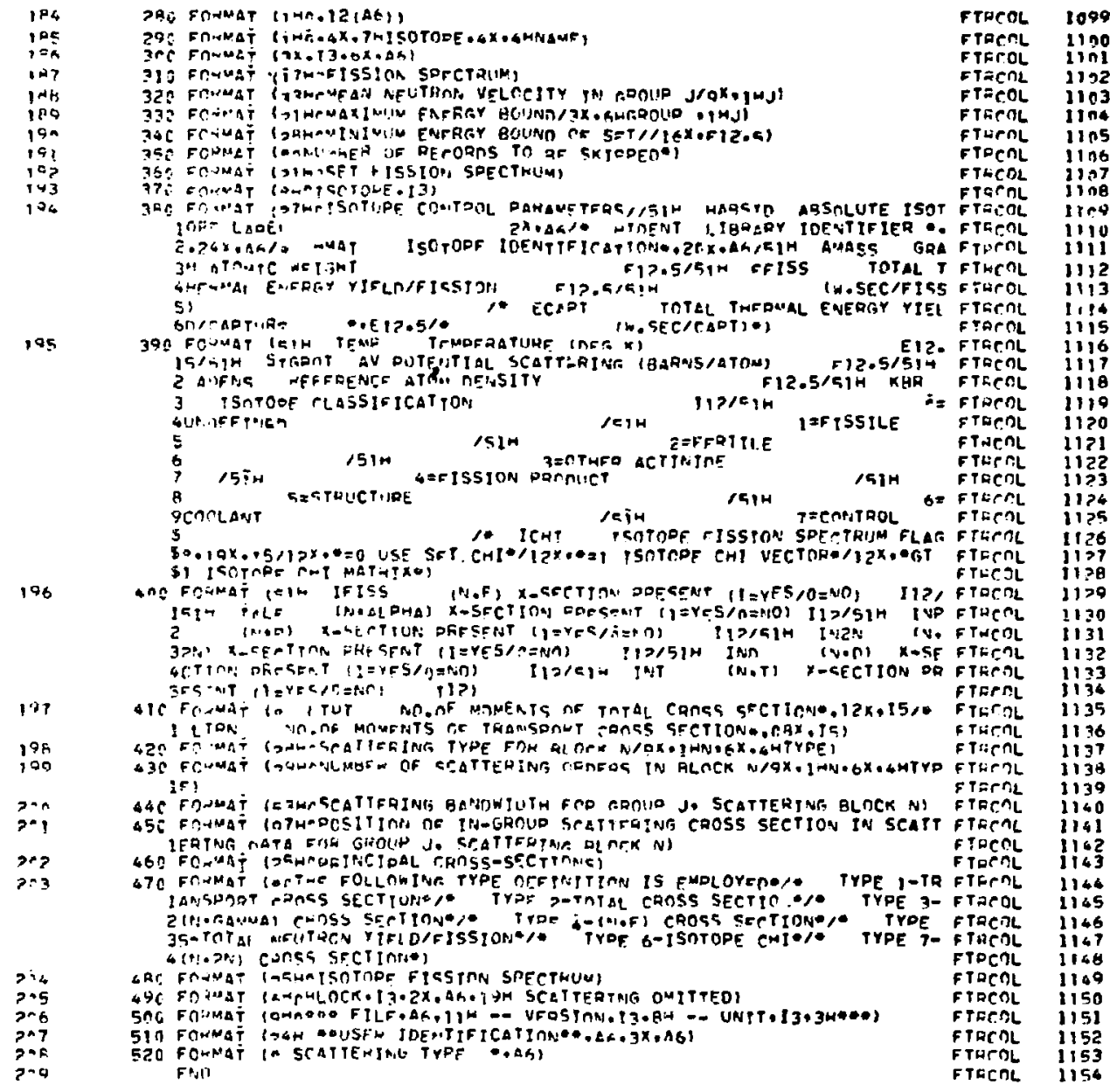

JanEX A2/J8/TS

SUAHOUTINE UISOTX IESYG. NIN]

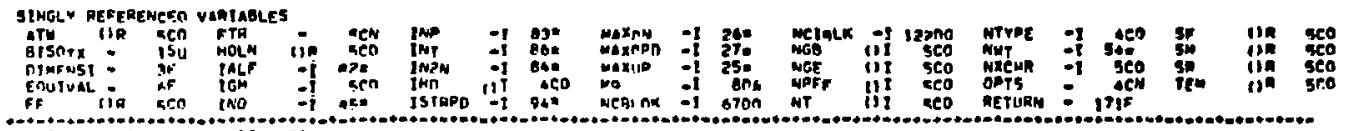

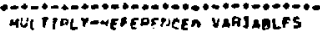

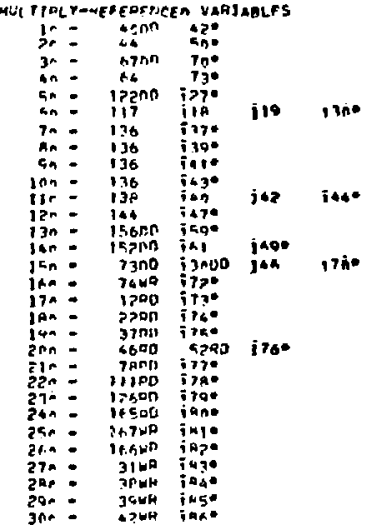




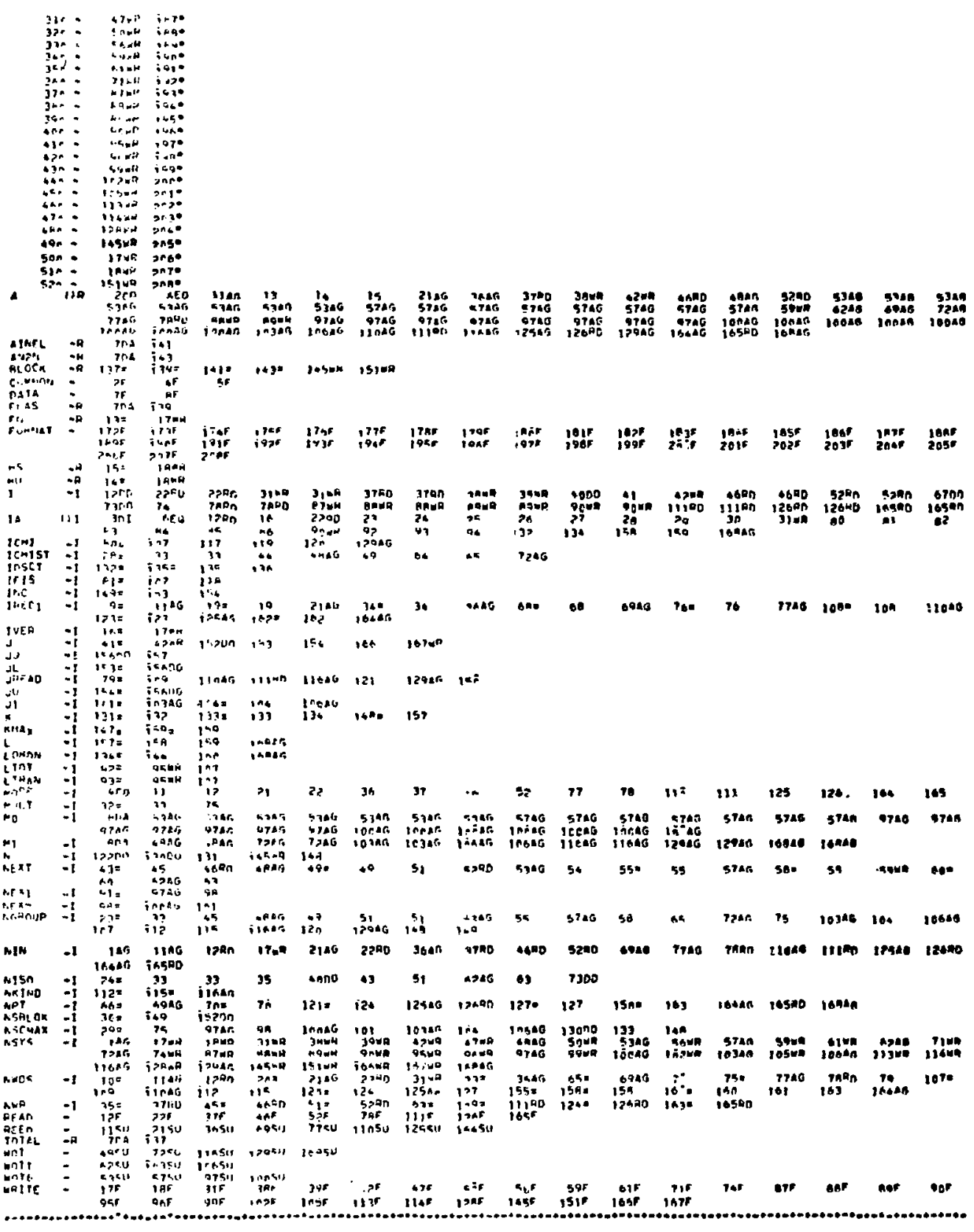




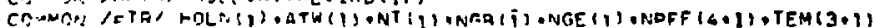

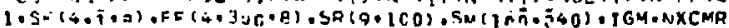

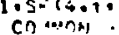

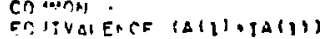

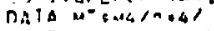

$\dot{r}$

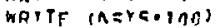

a-emit timentification

$[\sec C=i$

Bi. 1 . $15=4$

IF (MONE.FO.1) CALI HEFO (NIN-IREC).A(I) -NWOS.1)

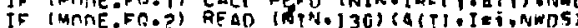

$F H_{1}=A(\bar{T})$

Hy=A $A, 3\}$

$H S=\Delta(9)$

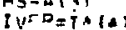

WDITF (NEYS.ABC)FN, ILEO,NIN

WFITE inERG.4lCituits

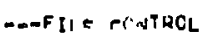

Iarel $=$ TReCl*1

$N A) S=4$

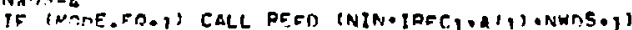

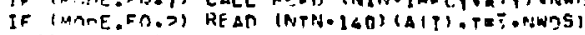

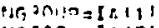

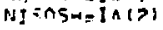

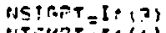

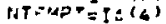

De. $17,=1,0$

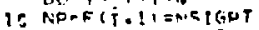

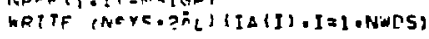

nofitr nata

NhH

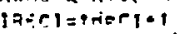

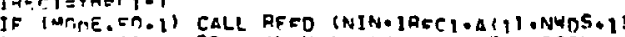

IF (IATRE,FO,

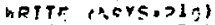

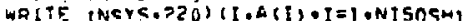

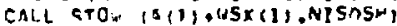

DERT

hätTE (AEYC.>3t)

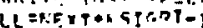

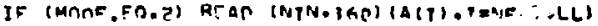

NM=?.

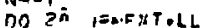

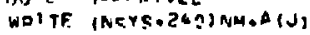

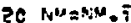

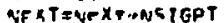

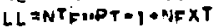

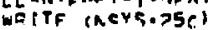

is $=1$

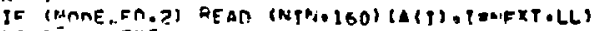

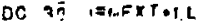

HEI TE INEYSODQR:NMALIJ)

30 M

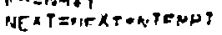

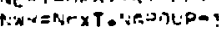

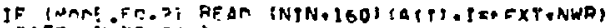

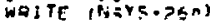

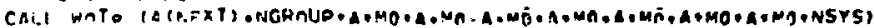

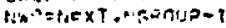

A.F.t

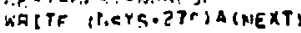

HIT $A 1=+15 x+1$

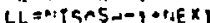

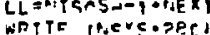

nimite

IF (WNEE,FA.P) HEO

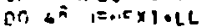

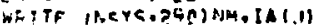

4: $: \cdots=0,4, i$

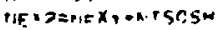

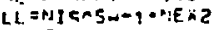

TEITE $1:=-5.3601$

$: \pi, 4=1$

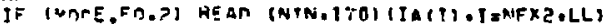

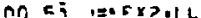

White incegossinnotalj)

ilsc

FTirni I1K

FTECOL 1162

FTFr.OL 1163

FTacol 1164

FTREOL 1165
FTPCOL
1166

FTACML 116 ?

FTECFL IIA5

$\begin{array}{ll}\text { FTFCOL } & 1159 \\ \text { FTFCOL } & 1170\end{array}$

fincol liti

FTREDL jitz

FTirnl 1173

FTarol 1175

FizenL 1176

FTACTL 1177

-TeCOL 7178

FTERl 1179

FTECXL $11: 0$

riocol lial

Fiacol lige
FTfent lias

atiene liat

FTECOL 1175

FTECOL lipe

frarel liat

Etaral jiga
fThtol ifas

fTwerl 1100

ftirol 1191

FTFCAL 1192

F)

FTicol 1196

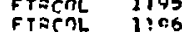

FTOCOL 1147

FToenl jiga

FTarel 1190

FTACAL IEnO

FTPCOL $1 \geq 0$

FTorne 1202

FTRCAL IPNA

fTacol 1205

fTarnL 3236

FTERAL 1207

FTDCTL I I RB

FTPCOL JENa

FTRCOL 1210

FTaCOL 1211

FTACOL 1212

FTPCOL 1213

FTFral 1214

FTOCAL 1215

Frecnl 1216

Frochl 1217

ETHCOL I2IS

FTFEnL $12 ? 0$

FTFCAL 1220
FTFTOL $12 ? 1$

fTFCX 1222

FTrene 12?3

$17 ?$

FTOCAL 3225
FTRCAL 1276

FTRPAL $12>7$

fTirta iz?o

Firen 1279

fterne 1270

fiarnl 1231

ctactr 1232

fiacnl 1233

FTECRL 1236

FTECAL 1235

Firen: 1236

FTatol 1237

FTRCAL 1239

FTACAL 1279

Fincal 1240

fTachl izaz 


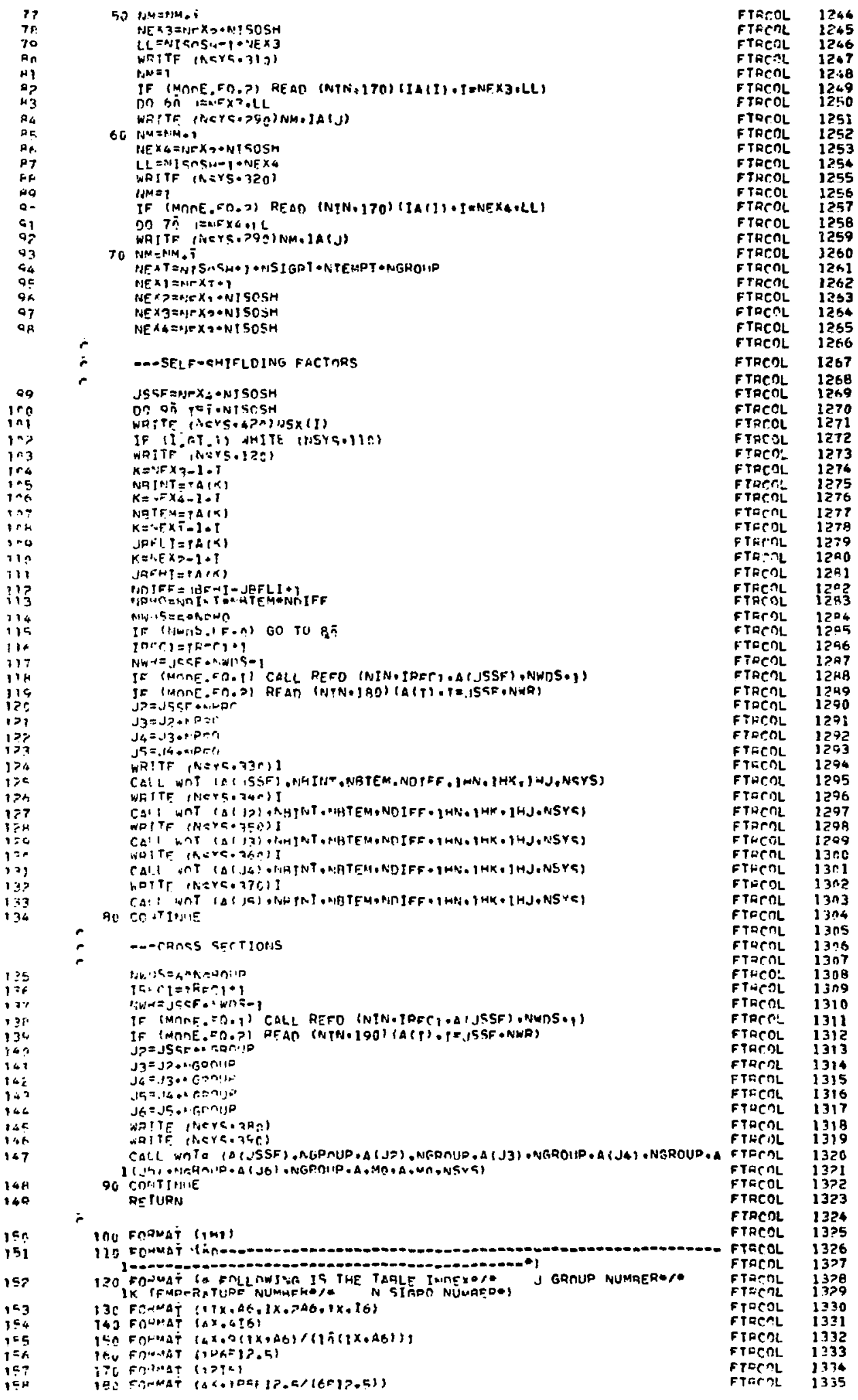




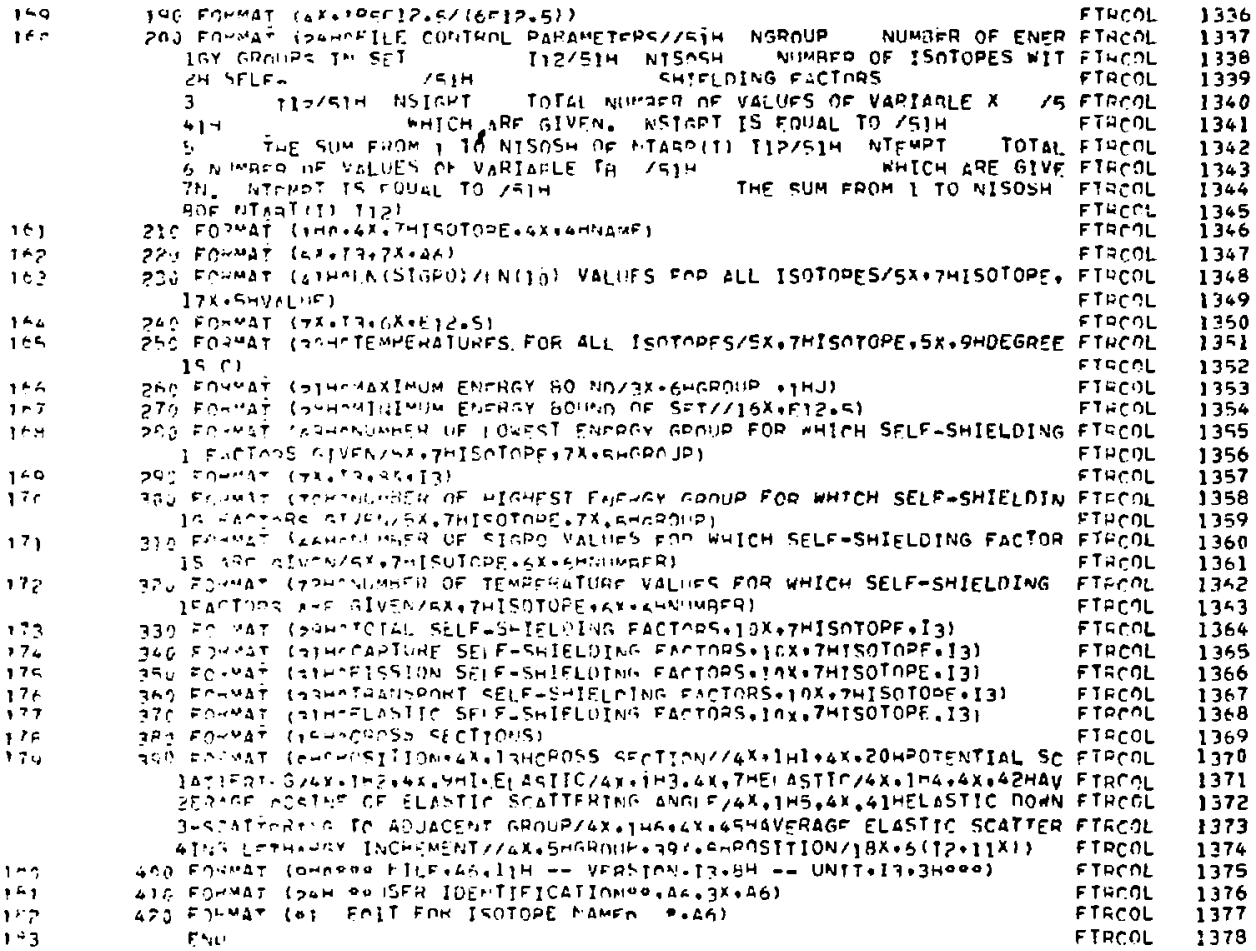

tarea 02/16/75

SUAKOUT IME GRAKEINSYS.NINI

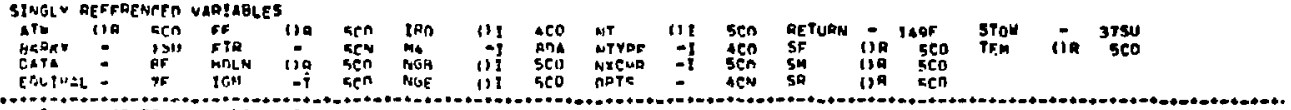

aripiolr-affepicen vahiagles

if: : lane $\rightarrow c=$

ye - cirn $45^{\circ}$

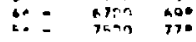

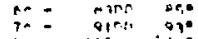

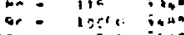

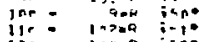

joe: irson ic?o

lia: lian is30

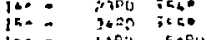

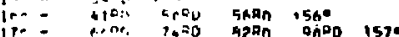

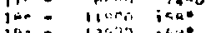

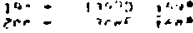

zir:

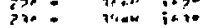

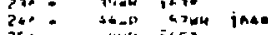

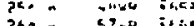

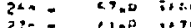

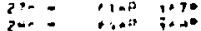

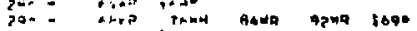

ine: ris irno

Jin: - and ino

ije- 1i4no ipo

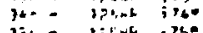

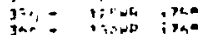

yat: iven ilat

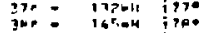

jun : room ipos

tia - lame inie

ar if lime inte

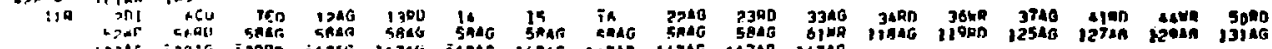

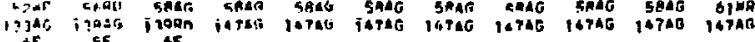

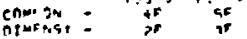




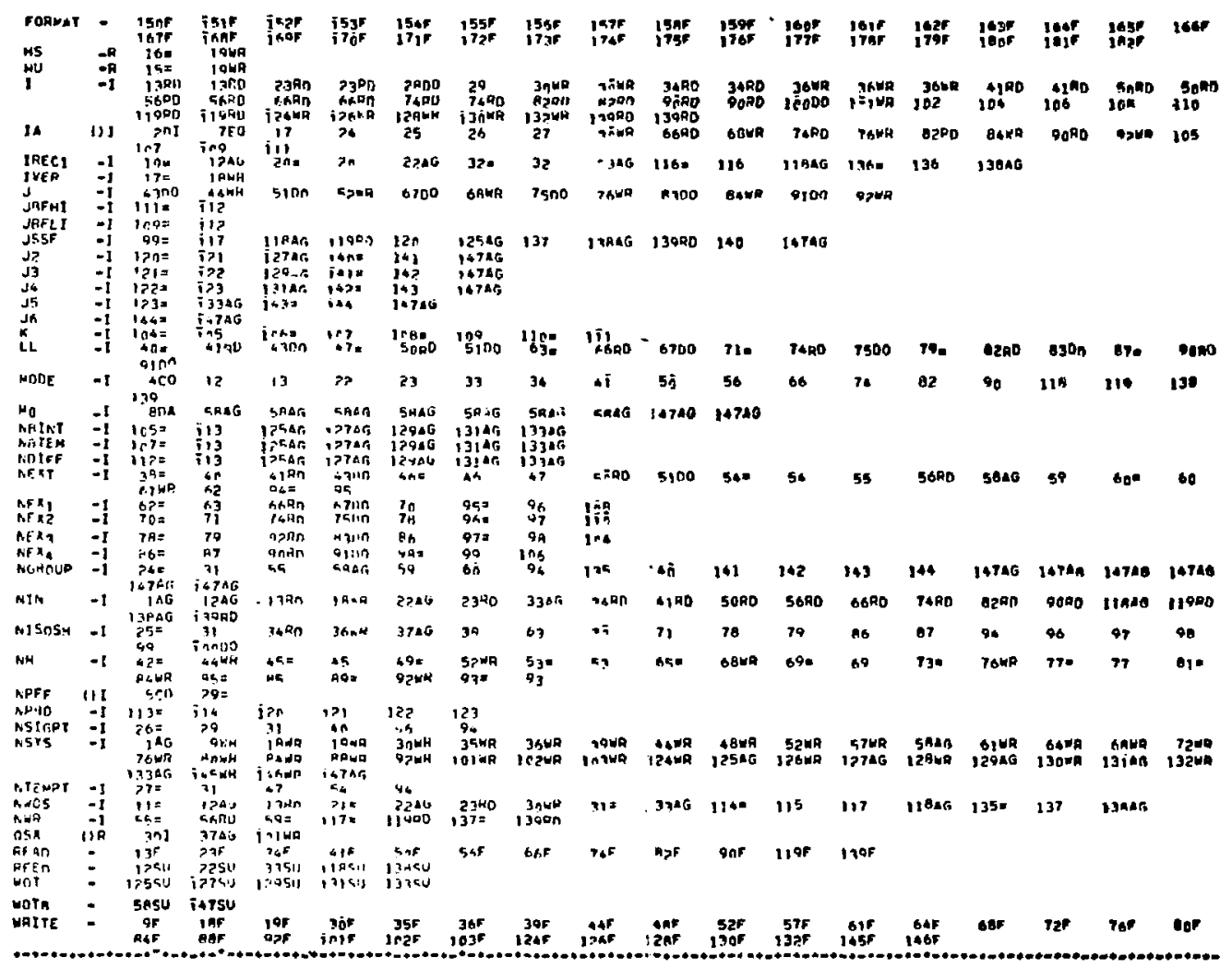

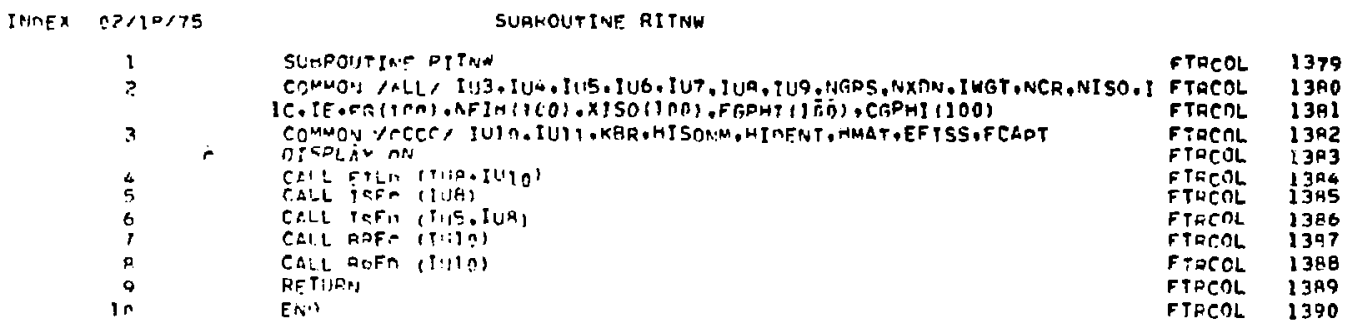

INAFA neCIANTS

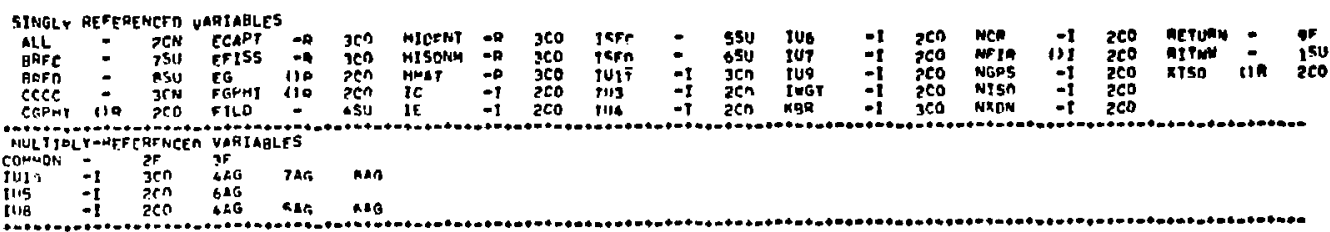




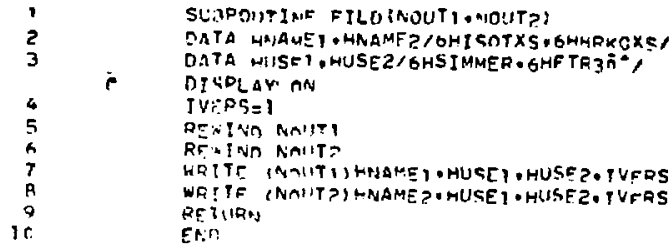

$\begin{array}{ll}\text { FTREOL } & 1391 \\ \text { FTRCOL } & 1392 \\ \text { FTPCOL } & 1393 \\ \text { FTACOL } & 1394 \\ \text { FTRCOL } & 1395 \\ \text { FTACOL } & 1396 \\ \text { FTOCOL } & 1397 \\ \text { FTPCOL } & 1396 \\ \text { FTRCOL } & 1399 \\ \text { FTACOL } & 1300 \\ \text { FTPCOL } & 1401\end{array}$

JHEEX AP/IR/75

SUFTOUTINE FILUINCUTI -NOUTZ)

SIAELY REFFPENCTO VARIABLES

FILO - jSII PETURN - QF

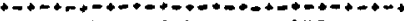

MUL TIELYANEFERTIEEN VARIARLES

CATA - JF JF

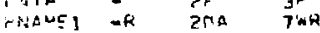

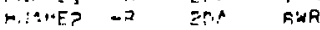

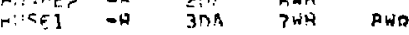

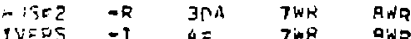

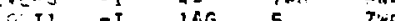

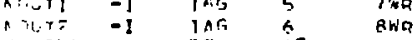

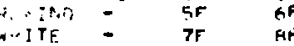

NITE

AF

\begin{tabular}{|c|c|c|c|c|}
\hline NnF $x$ & $n ? / 19 / 75$ & SUAKOLTIHE ISFC(THOUT) & & \\
\hline & 1 & SUTROUTIUF ISFC(NOUT) & F ToCOL & 1402 \\
\hline & 2 & 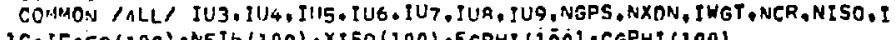 & FTPCOL & 1403 \\
\hline & f & 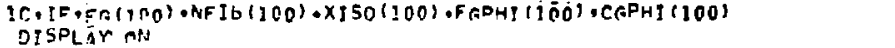 & $\begin{array}{l}\text { FTOCOL } \\
\text { FTFCOL }\end{array}$ & $\begin{array}{l}1404 \\
1405\end{array}$ \\
\hline & 3 & $\begin{array}{l}\text { Nisx }=8 \\
\text { MnXUP=- }\end{array}$ & $\begin{array}{l}\text { FTHCOL } \\
\text { FTHCOL }\end{array}$ & $\begin{array}{l}1406 \\
1407\end{array}$ \\
\hline & 5 & $\begin{array}{l}H_{A} \times \cap R_{n}=0 \\
I C+I G T=0\end{array}$ & $\begin{array}{l}\text { FTACAL } \\
\text { FTECNL }\end{array}$ & $\begin{array}{l}1408 \\
1409\end{array}$ \\
\hline & 7 & $\operatorname{tg} \operatorname{sen} \Delta x=1$ & F tPCOL & 1410 \\
\hline & R & $A S-L D K=N C H$ & FTACOL & 1 \\
\hline & 9 & WBITF (NOIJT)NCA,NISA, MAXUP,NCR,MAXORD,TEHIST,NSCHAX,NSBLOK & FTRCOL & 12 \\
\hline & 3 & RETUPA & F TPCOL & 1613 \\
\hline & i! & Enil & FTACOL & $1 4 \longdiv { 1 6 }$ \\
\hline
\end{tabular}

\begin{tabular}{|c|c|c|c|c|c|c|c|c|c|c|c|c|c|c|c|c|c|}
\hline$I \cap \cap F$ & $F x \quad 5$ & $\mid 1 R / 75$ & & & & su & TINF & ISFC & ? & & & & & & & & \\
\hline SINELY & AEFFW & ENeFn & $\checkmark \triangle P\{A B L E$ & & & & & & & & & & & & & & \\
\hline $\begin{array}{l}\text { ALL } \\
\text { CFFH: } \\
\text { CRNHAN } \\
\text { ER }\end{array}$ & $\underset{110}{i a}$ & $\begin{array}{l}\text { PCin } \\
\text { Pron } \\
\text { PF } \\
\text { Pin }\end{array}$ & $\begin{array}{l}\text { FRTMI } \\
\text { IE } \\
\text { IE } \\
\text { ISFC }\end{array}$ & $\begin{array}{l}138 \\
-7 \\
=7 \\
=\end{array}$ & $\begin{array}{l}\text { Per } \\
\text { Per } \\
\text { sen } \\
\text { isin }\end{array}$ & $\begin{array}{l}I U_{3} \\
\text { IU4 } \\
\text { IUS } \\
\text { IUt }\end{array}$ & $\begin{array}{l}-1 \\
-1 \\
-1 \\
-1\end{array}$ & $\begin{array}{l}200 \\
200 \\
200 \\
200\end{array}$ & $\begin{array}{l}\text { TUT } \\
\text { TUA } \\
\text { TI19 } \\
\text { IWET }\end{array}$ & $\begin{array}{l}-1 \\
-1 \\
-1 \\
-1\end{array}$ & $\begin{array}{l}2 c n \\
26 n \\
2 c n \\
2 c n\end{array}$ & $\begin{array}{l}\text { NF I } \\
\text { NGPS } \\
\text { NISB } \\
\text { NXDN }\end{array}$ & $\begin{array}{l}1) 1 \\
-1 \\
-I \\
-1\end{array}$ & $\begin{array}{l}P C 0 \\
P C O \\
? C 0 \\
>C 0\end{array}$ & $\begin{array}{l}\text { RETUAN } \\
\text { W91:E } \\
\times 150\end{array}$ & $\overrightarrow{-}$ & $\begin{array}{l}10 F \\
9 F \\
2 C 0\end{array}$ \\
\hline MLLTI"L & $y=-2$ & HFticE & $V A+I A A$ & $=-\cdots$ & & & & & & & & & & & & & \\
\hline Ir-!st & $-I$ & $f=$ & $\operatorname{ans}$ & & & & & & & & & & & & & & \\
\hline$\omega a x c=0$ & -1 & $5 x$ & $94 \mathrm{H}$ & & & & & & & & & & & & & & \\
\hline$N A X t, P$ & -1 & $4=$ & op. & & & & & & & & & & & & & & \\
\hline$A C \bar{H}$ & $-I$ & $p e^{n}$ & P & OHD & and & & & & & & & & & & & & \\
\hline$n I \leq x$ & $-i$ & $3=$ & QWA & & & & & & & & & & & & & & \\
\hline A V T & -1 & $j \propto 5$ & ghH & & & & & & & & & & & & & & \\
\hline$\because \therefore \angle O K$ & $-i$ & $A=$ & QWR & & & & & & & & & & & & & & \\
\hline$n=\cos 3 x$ & $-I$ & $7=$ & 944 & & & & & & & & & & & & & & \\
\hline
\end{tabular}


INDEX $03 / 25 / 75$

SUBROUTINE ISFO(NIN, NOUT)

PAGE 69

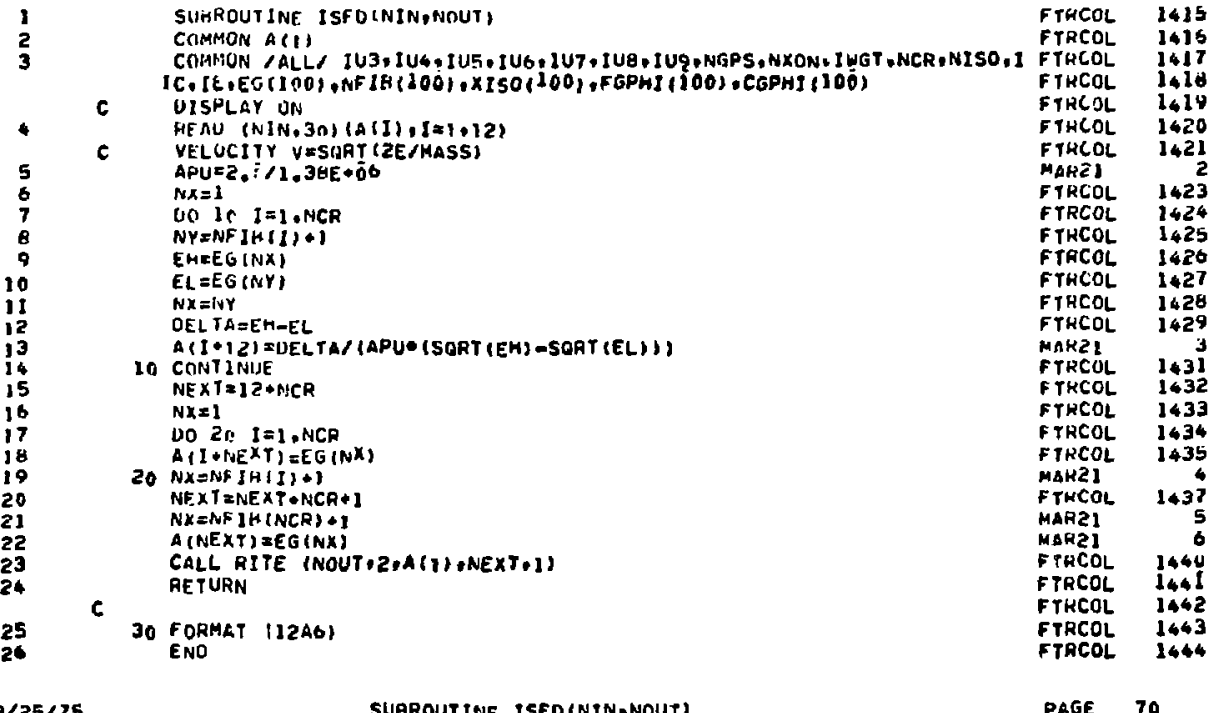

IMOEX C3/25/7S

SUBROUTINE ISFDININ,NOUTI

PRGE 70

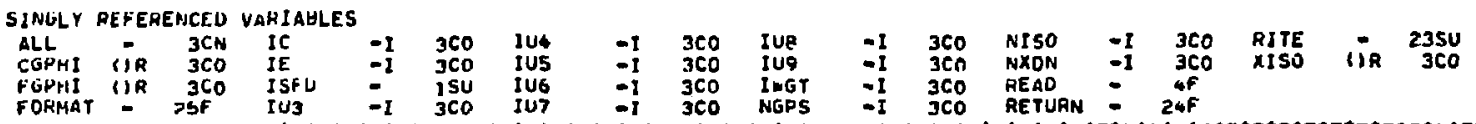
FORHAR

MULTIPLY-QEFEPENCEO VARIABLES

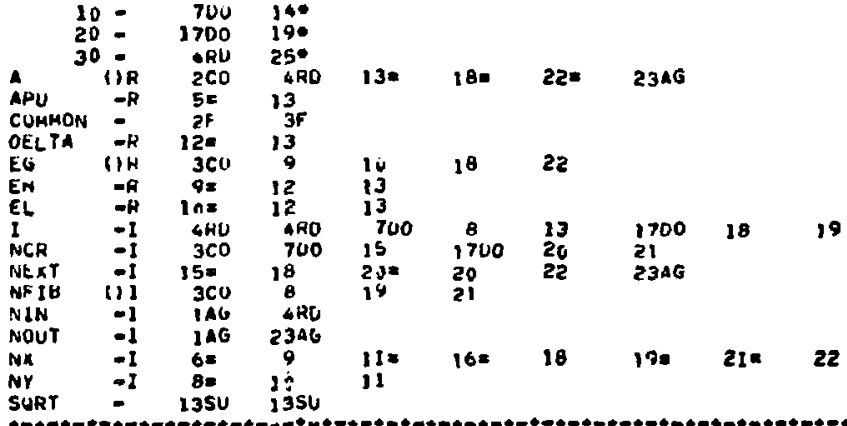

INAEX APSIMSTS

SURHOUTINE ARFE(HNIT)

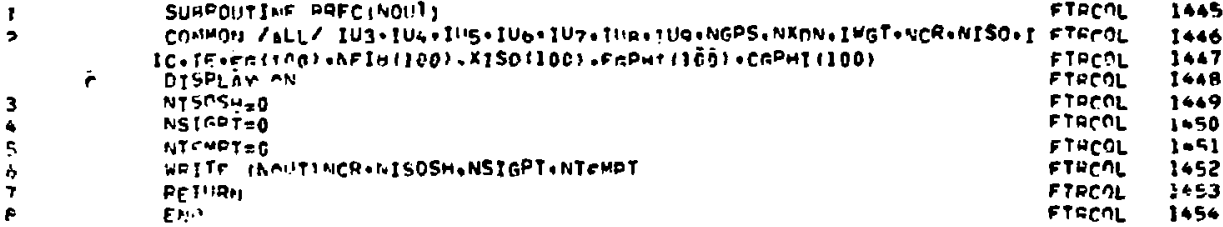

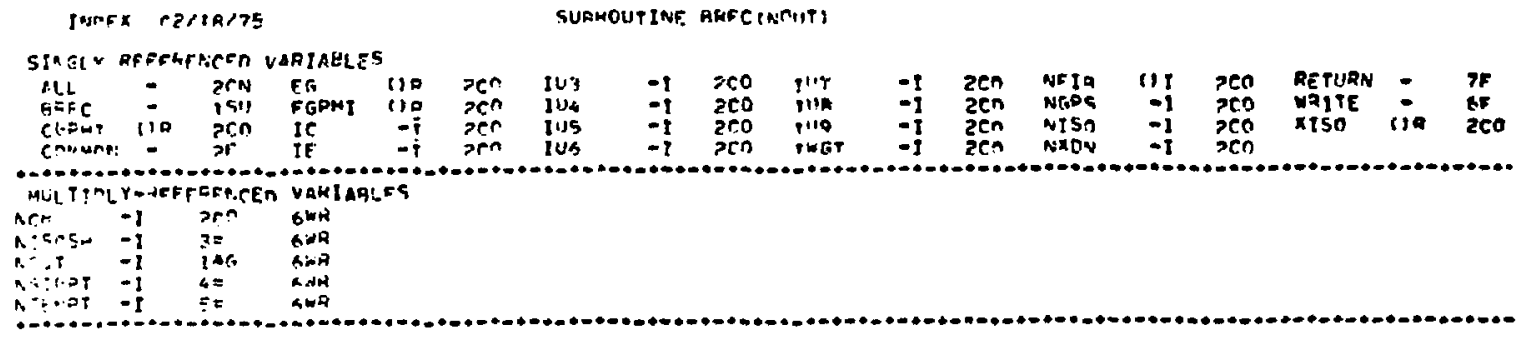




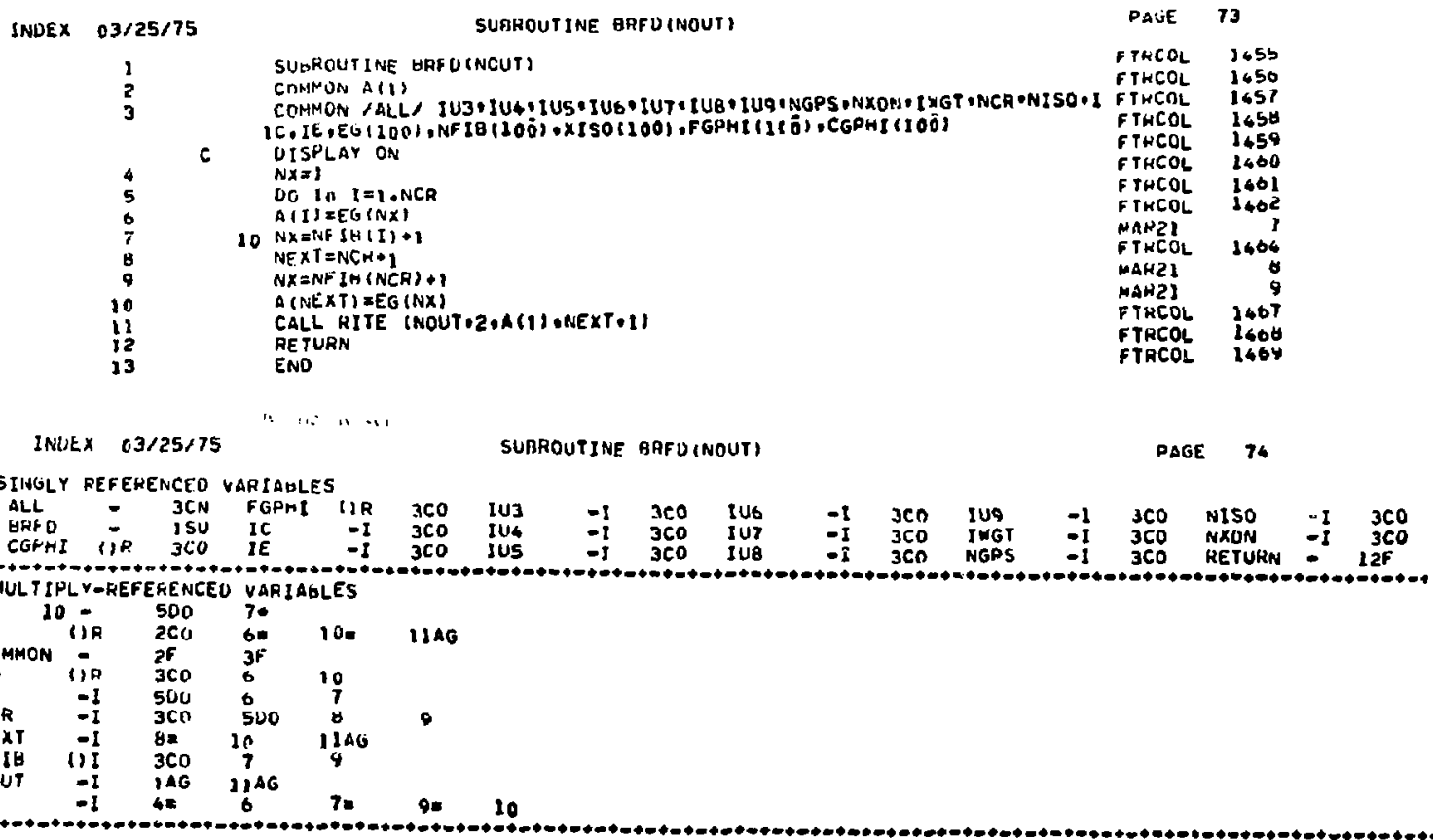

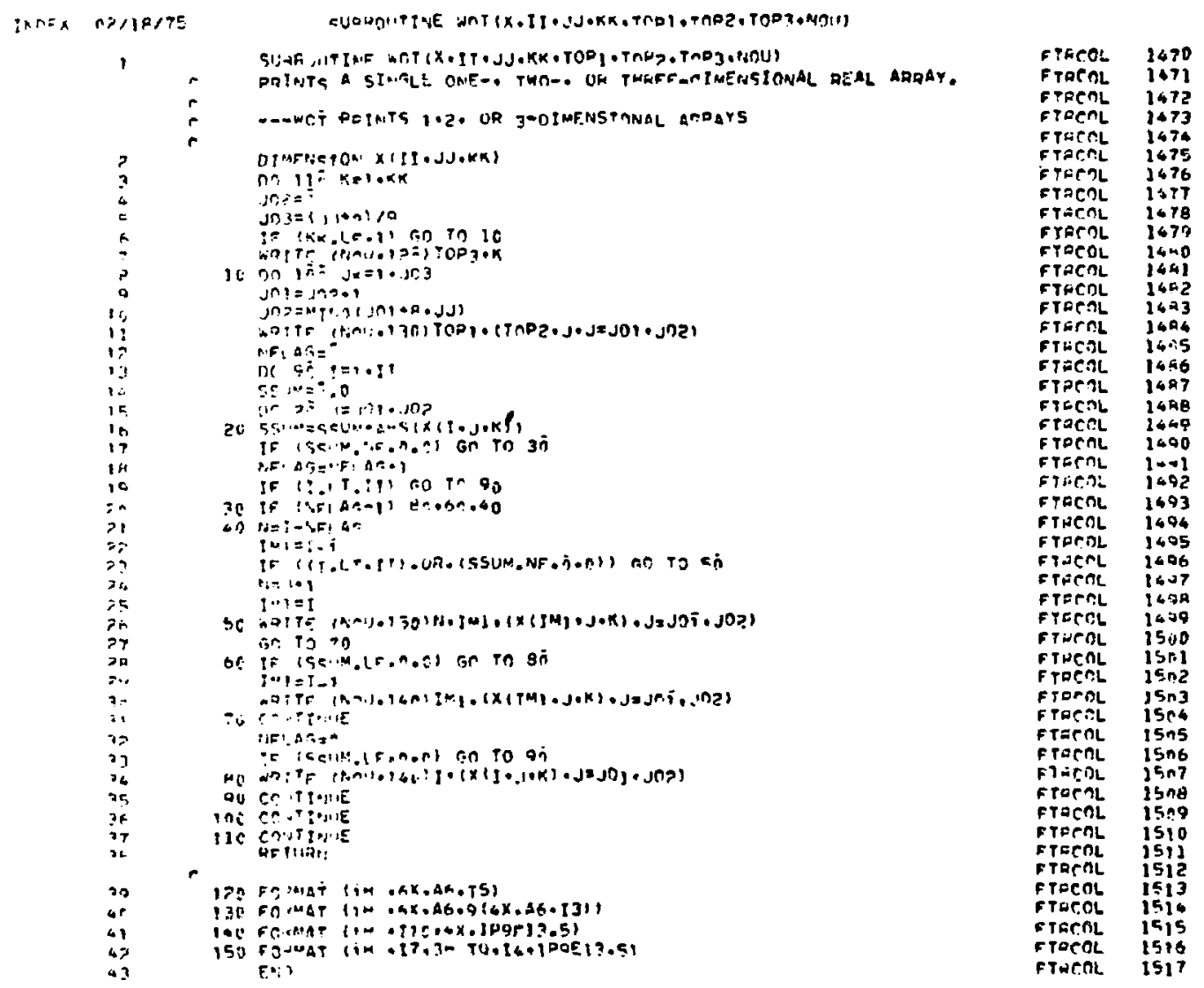




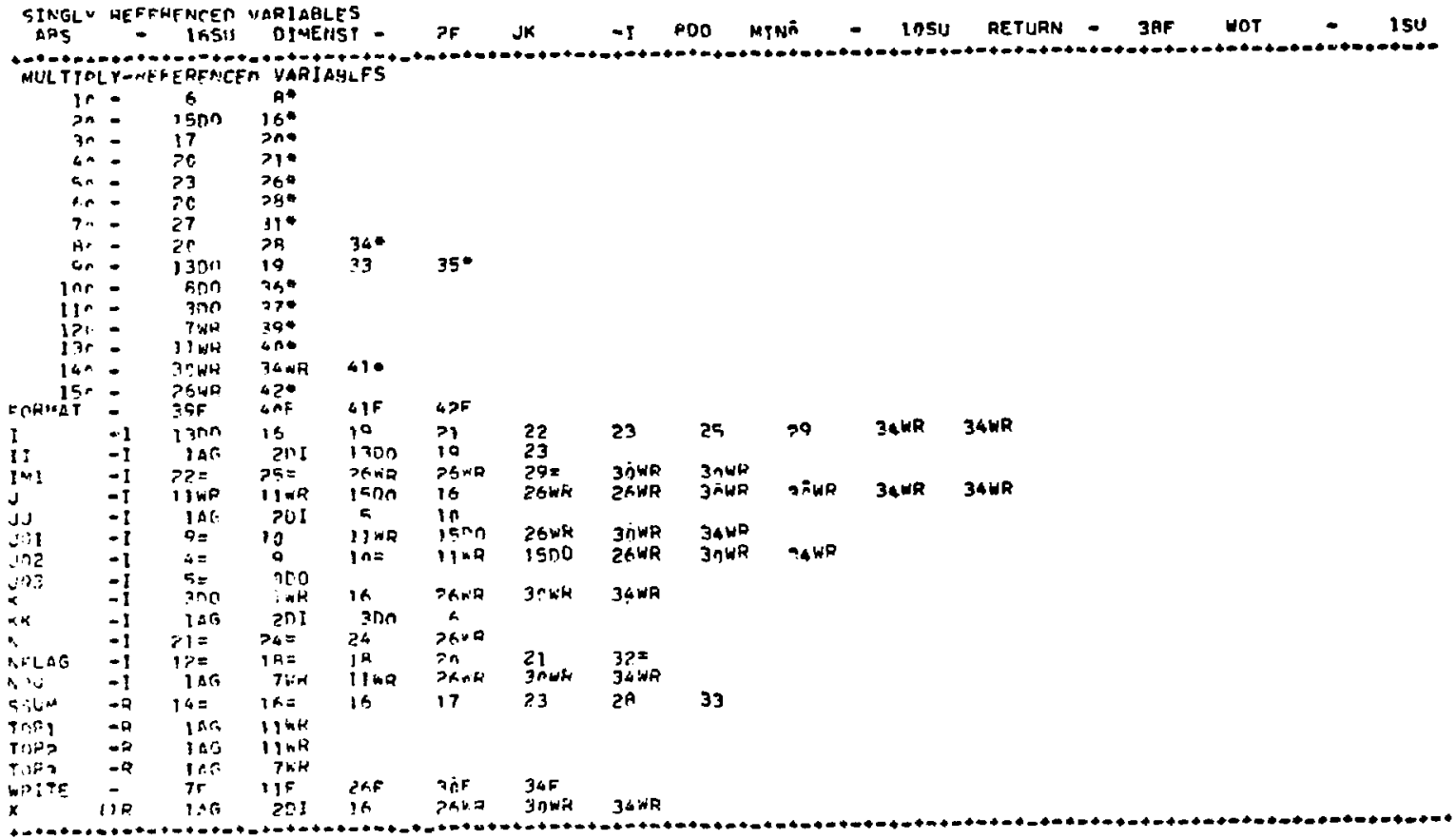

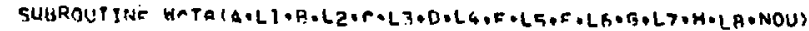

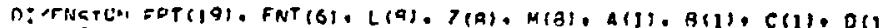
1). F(F), $(1) \cdot G(1) \cdot H(1)$

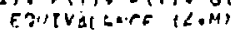

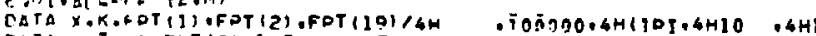

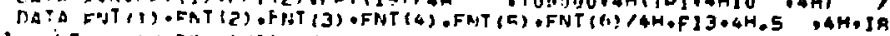

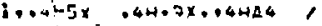

Li!

$[(2)=1 ?$

I. (3) $=17$

$L(i)=14$

$L(5)=15$

$L(4)=15$

$L(A)=0$

$\mathrm{H}=\mathrm{r}$.

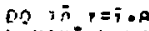

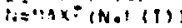

10 COATI*ine

5i: $15=1510 *$

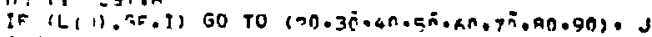

I $11=x$

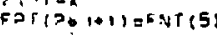

EDT $(2 क, 102)=F N T(6)$

Git in il

20 $7(.1)=411$

an in in:

30 $21:=011$

is in ing

4C $7(1)=0 ! ! !$

कo

$5212=011$

or To ig:

SC $7-6 \mathrm{~s})$

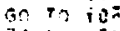

(c) $2 ! j=5$ II

(i) 1 (1) $=5,1$ if

की Tr

9c ?. $11=w$ :

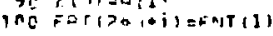

\begin{tabular}{|c|c|}
\hline FTACDL & \\
\hline $\begin{array}{l}\text { FTPCDL } \\
\text { F TACOL }\end{array}$ & \\
\hline $\begin{array}{l}\text { ETRCX } \\
\text { FTRCnL }\end{array}$ & \\
\hline ETachl & \\
\hline $\begin{array}{l}\text { FTRCOL } \\
\text { FTRCOL }\end{array}$ & \\
\hline FTRCAL & \\
\hline FTRCAL & \\
\hline $\begin{array}{l}\text { FTARחL } \\
\text { FTaCחL }\end{array}$ & \\
\hline FTHCDL & \\
\hline FTOCOL & \\
\hline FTACAL & \\
\hline $\begin{array}{l}\text { FrornL } \\
\text { FTent }\end{array}$ & \\
\hline FTPCOL & \\
\hline FTFCOL & \\
\hline $\begin{array}{l}\text { FTECOL } \\
\text { FTHEOL }\end{array}$ & \\
\hline FTerne & \\
\hline FTACOL & \\
\hline FTERAL & \\
\hline FTHCDL & \\
\hline F THEDL & \\
\hline F TfrOL & \\
\hline FTECAL & \\
\hline $\begin{array}{l}\text { FTPCOL } \\
\text { FTUEOL }\end{array}$ & \\
\hline FrCOL & \\
\hline FTREAL & \\
\hline FioenL & \\
\hline F TarnL & \\
\hline $\begin{array}{l}\text { Ftare } \\
\text { ftare }\end{array}$ & \\
\hline $\begin{array}{l}\text { TARML } \\
\text { TACAL }\end{array}$ & \\
\hline & \\
\hline & \\
\hline $\mathrm{FC}$ & \\
\hline & \\
\hline 10 & \\
\hline
\end{tabular}




\begin{tabular}{|c|c|c|c|}
\hline $\begin{array}{l}41 \\
48 \\
43 \\
44 \\
46 \\
45 \\
47 \\
4= \\
40 \\
=6\end{array}$ & $\begin{array}{l}110 \\
123\end{array}$ & 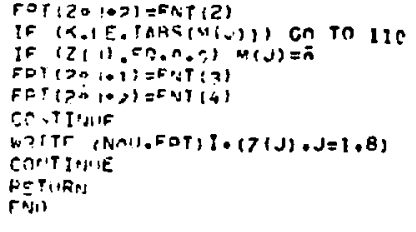 & 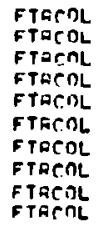 \\
\hline
\end{tabular}

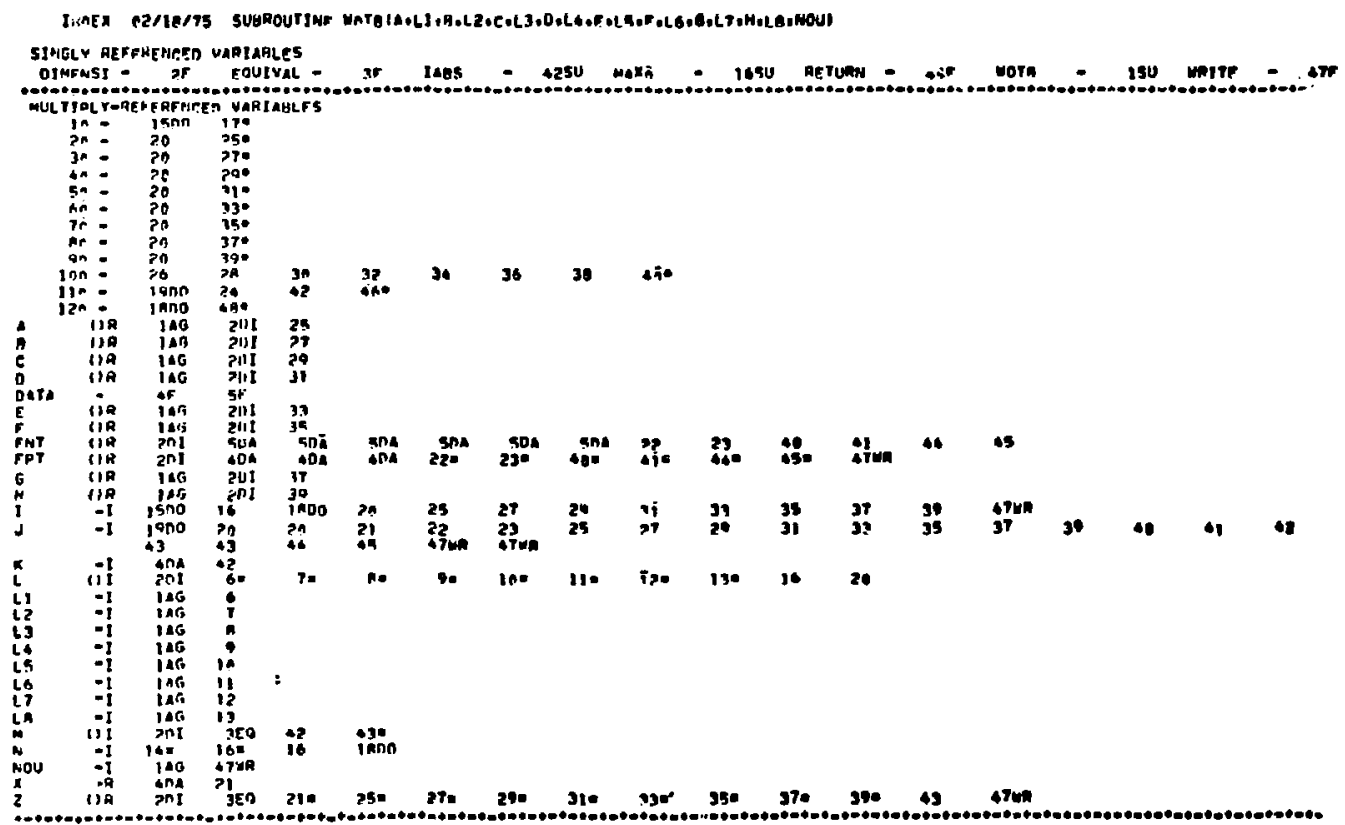

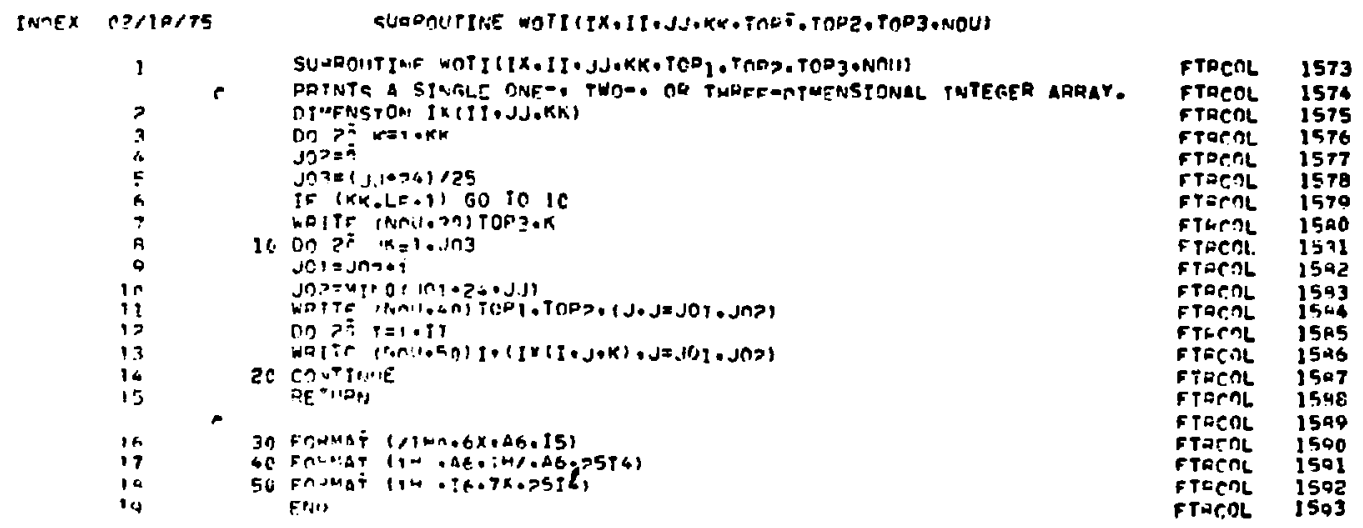




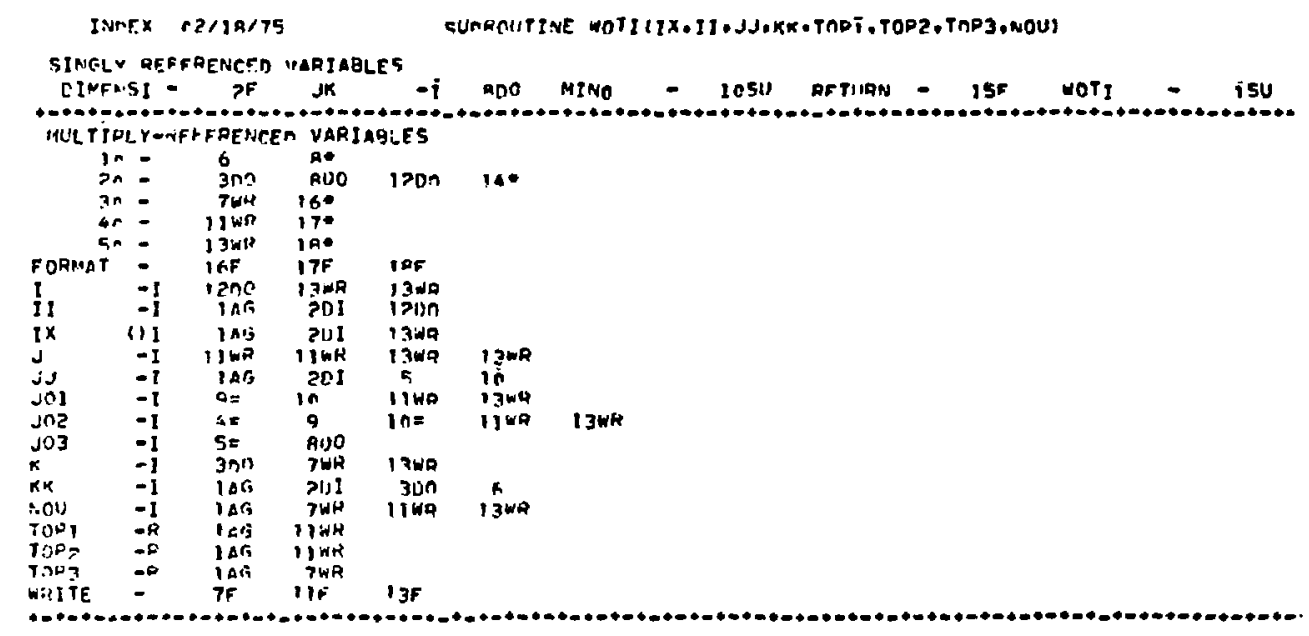

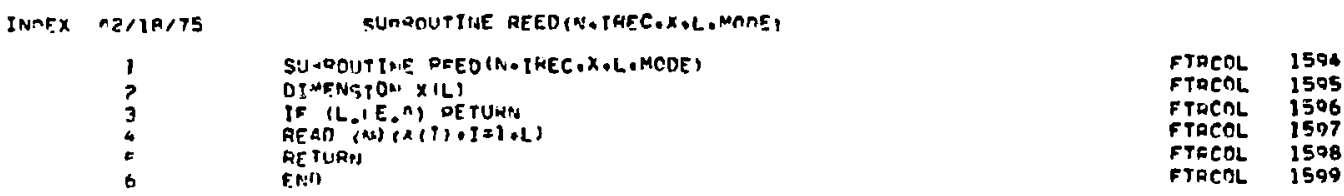

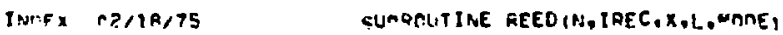

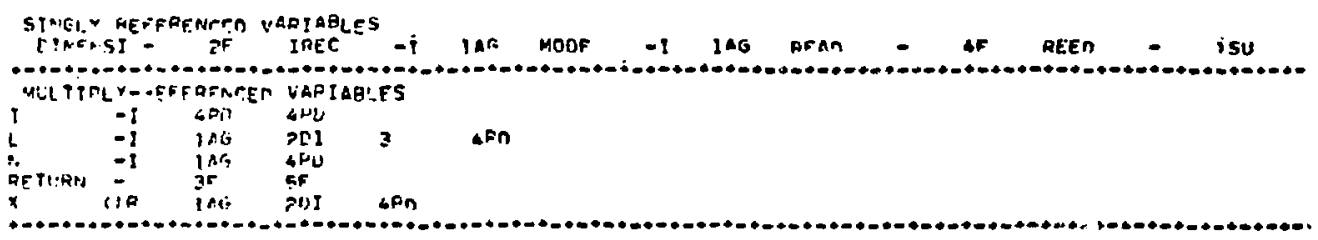

index cr/12/7E

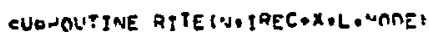

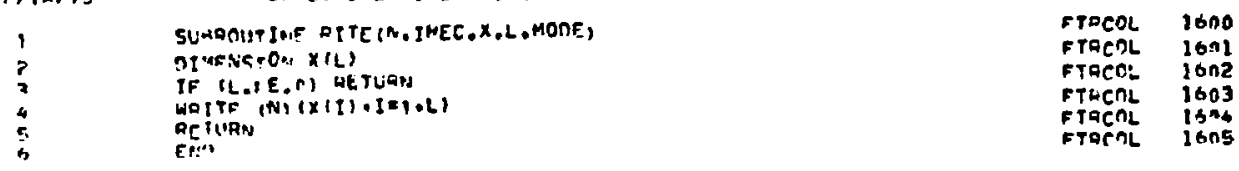

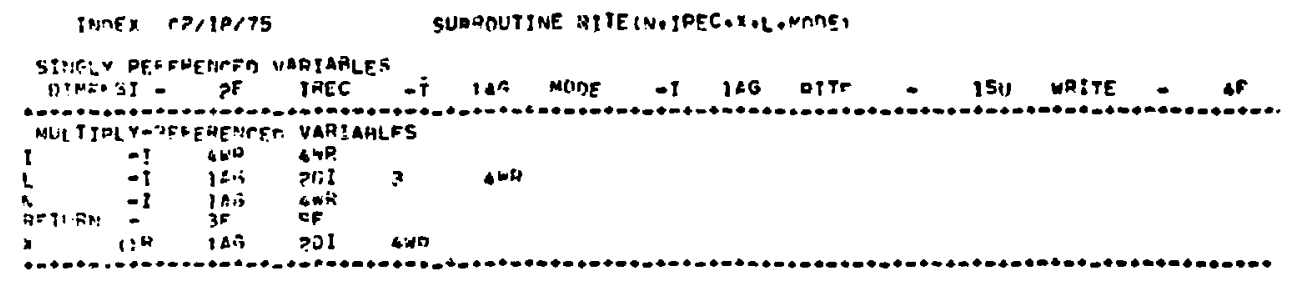




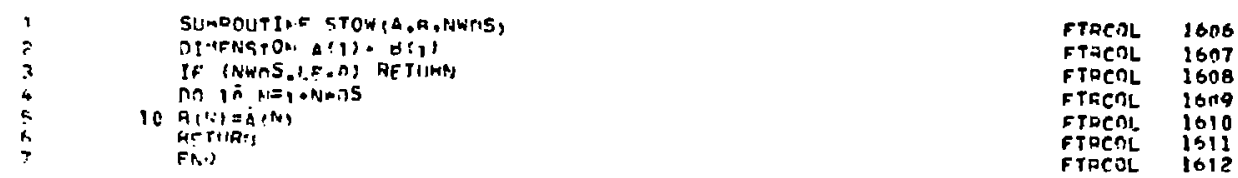

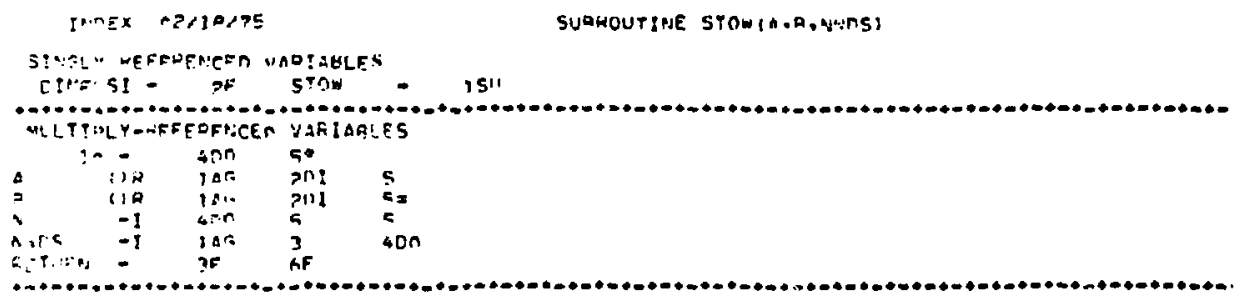

INEE C.7RID/75

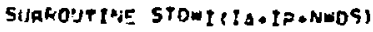

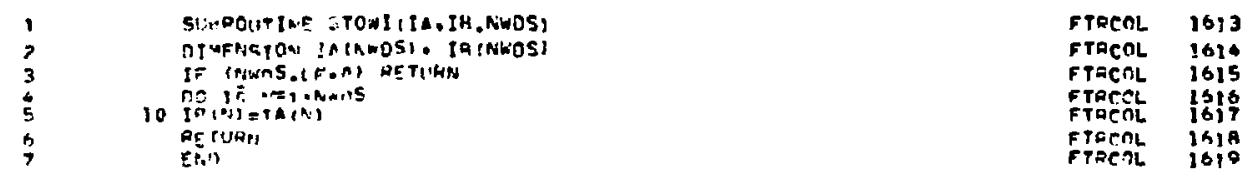

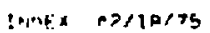

SUAKOUTINE STOWIIIA-IOANWOSI

SI:HL PEERZFHAFR YOOTAHLES

DISF.SI - PF STENT - ISI

1

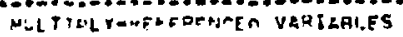

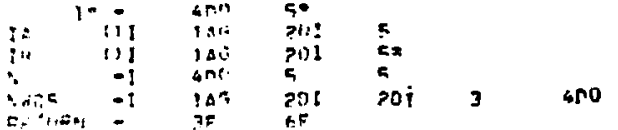

C.

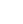

Jut Fa nosiarts

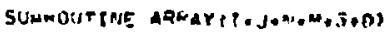

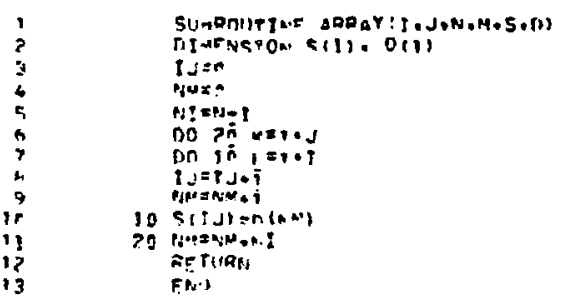

\begin{tabular}{|c|}
\hline 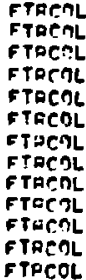 \\
\hline
\end{tabular}




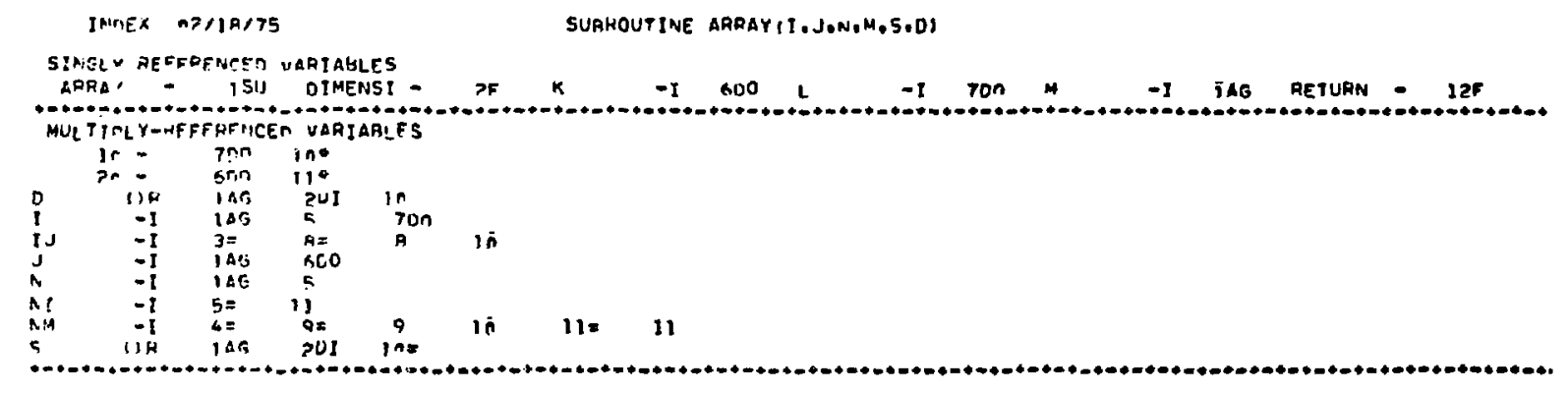

Iung: 02110/7s

masten ImEE

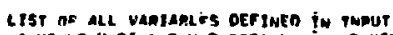

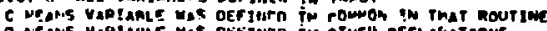

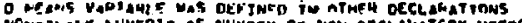

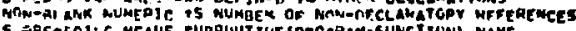

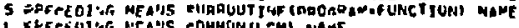

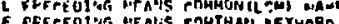

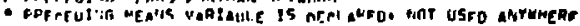

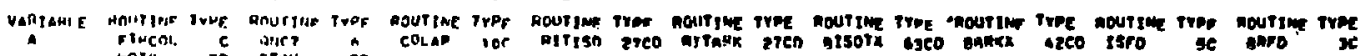

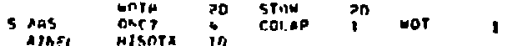

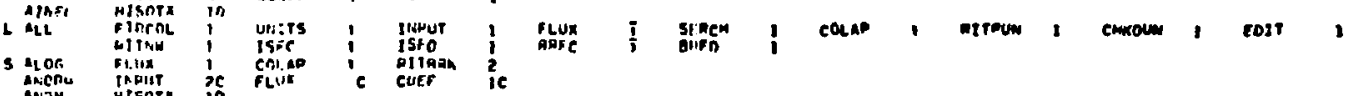

anichu in iplit

apu rasn

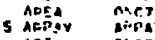

and ant

A7. Firrol

heckape stres

5 hanky
5 pitit

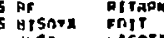

rioco misota

Mere.

hata

3 cref atice

eronar ilicos

chisa oljoint

s caintian

colea cliten

ce core

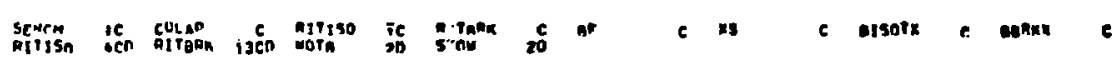

Ganax

Ge

hase

(19)

cnilís

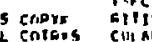

Hifan

hitisn

aphar in

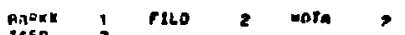

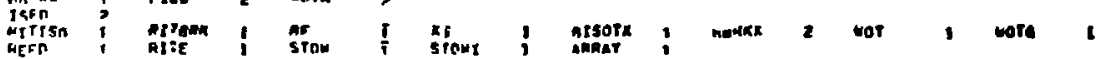

Crit

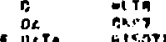

Drtinge Pritat

D. Gir

Dxt ant to

cespr atecol

ceut japut

sentr cmopul ic

En

chiso

Chiten fistix

El tes inf

Etas Hirints

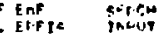

Eos tost Ginet

- Enyival oifiso

esi ance

este anct

ETfun birl zo

Exp lipur

catenena tolet

it wer op

frL

frap anc?

ire ripul rigen

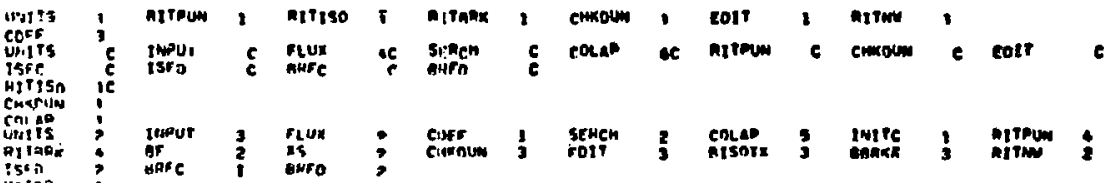

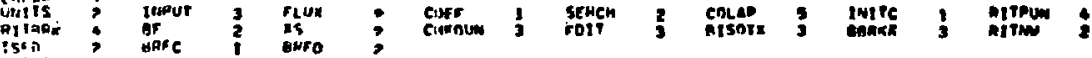

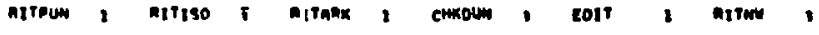

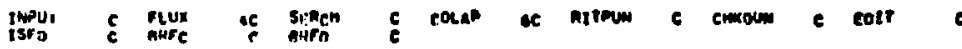

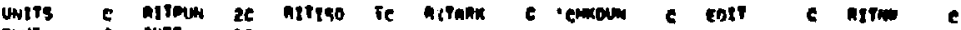

Elua e cuer

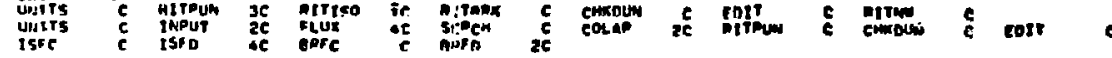

rlux, coef

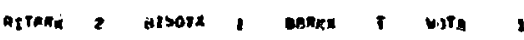

tiur C cluer it

cose 1

vore in

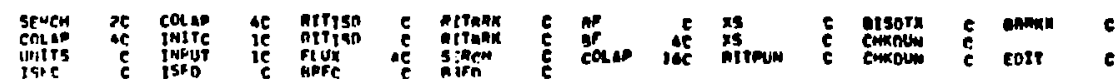




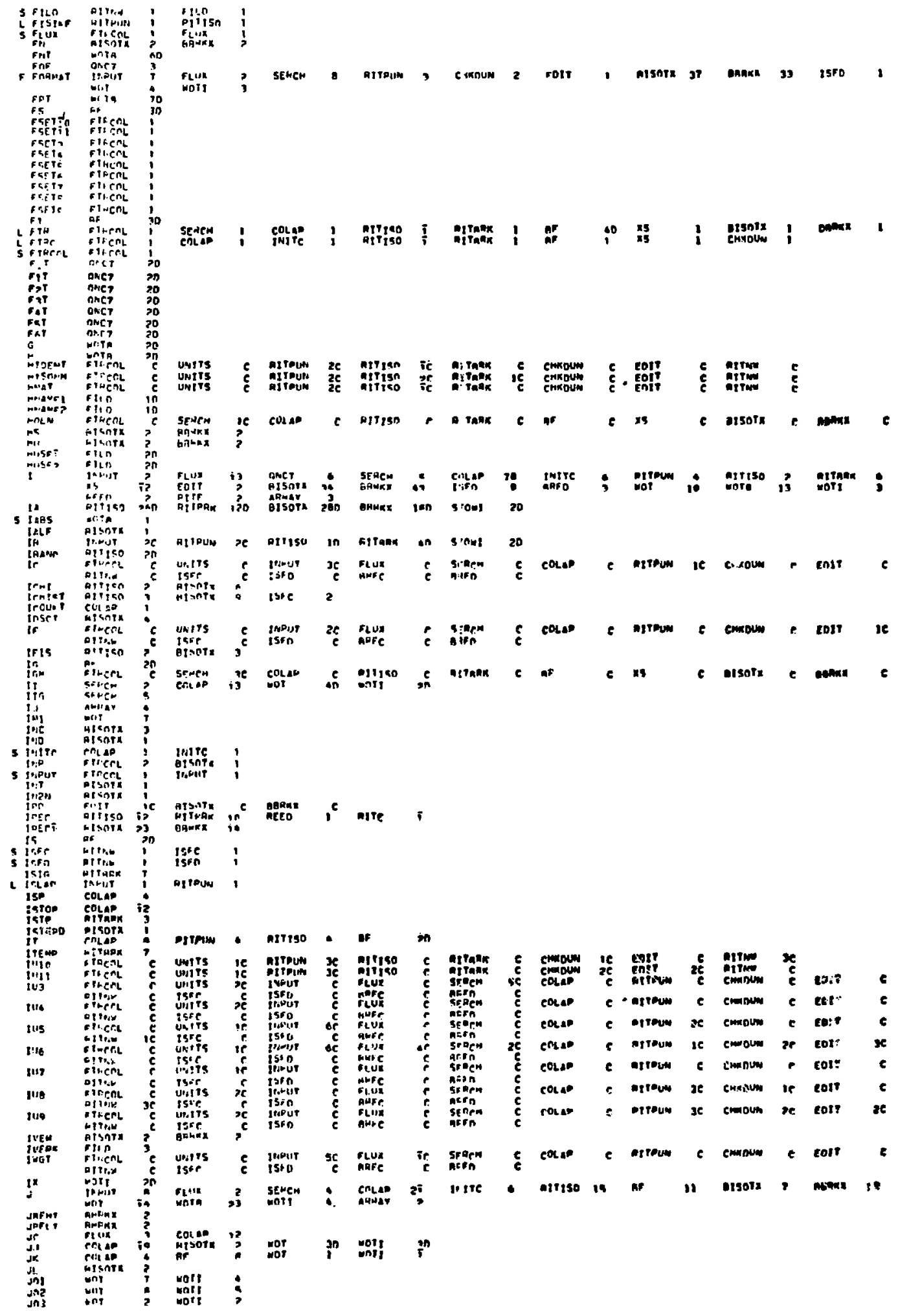




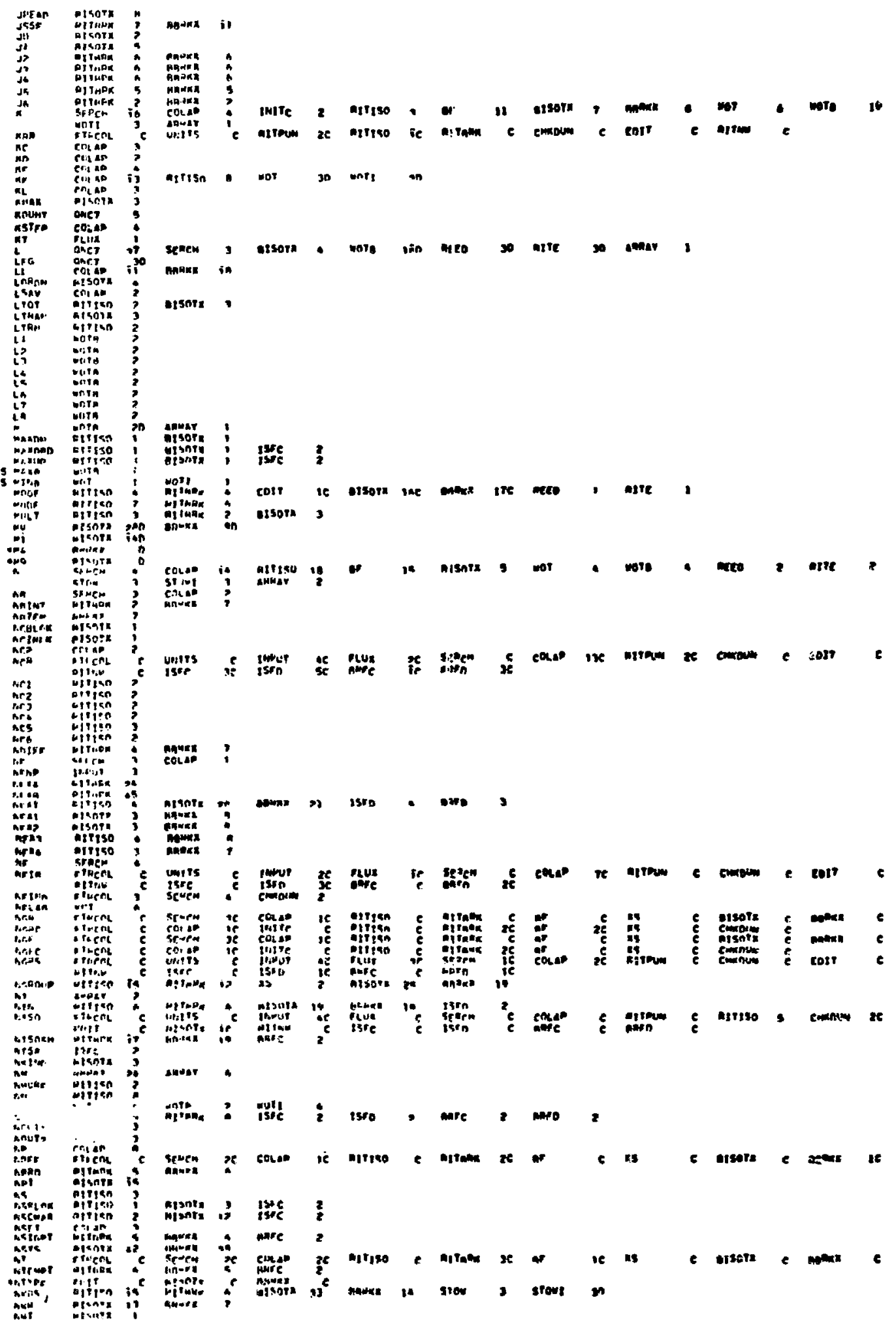




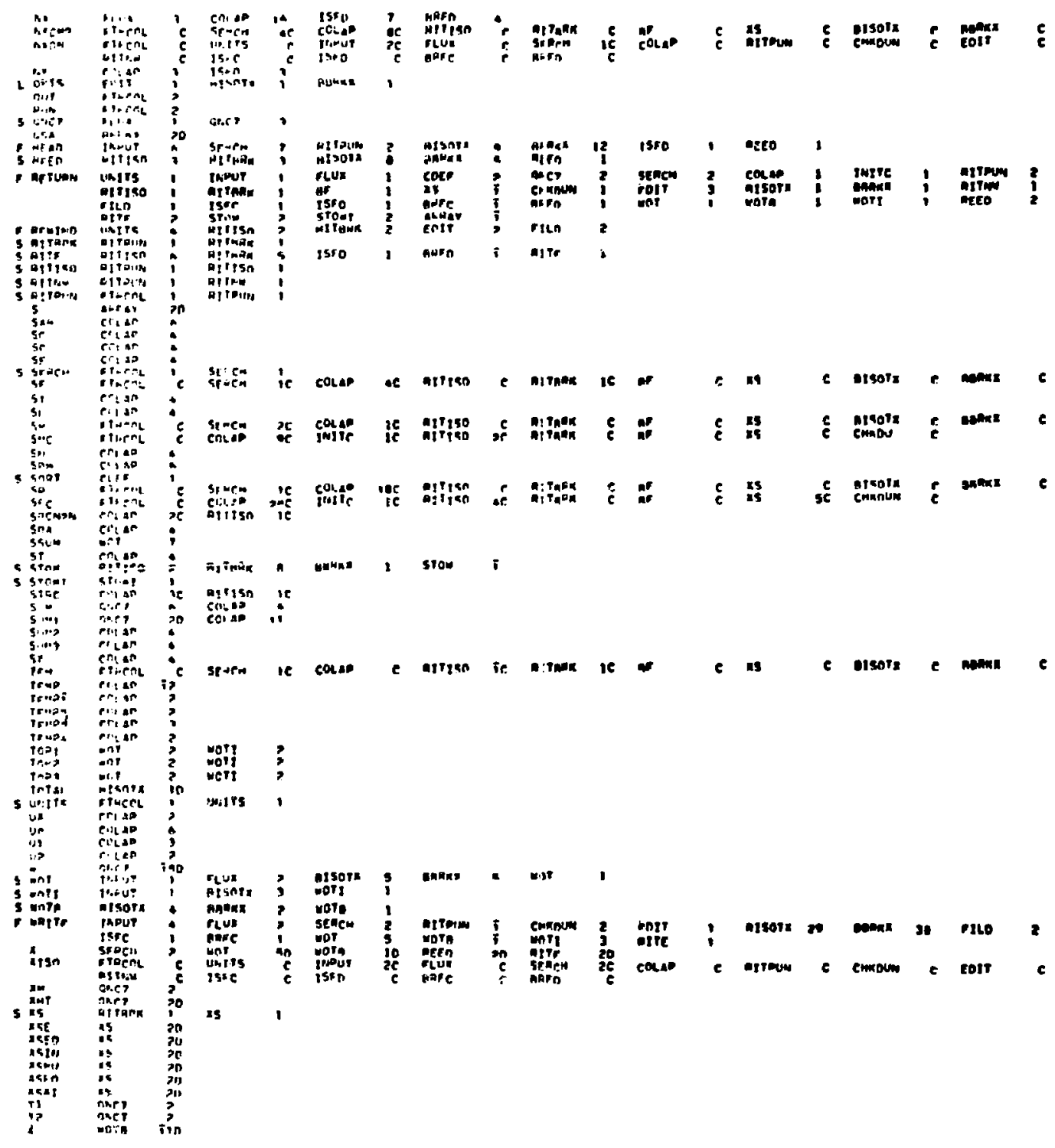

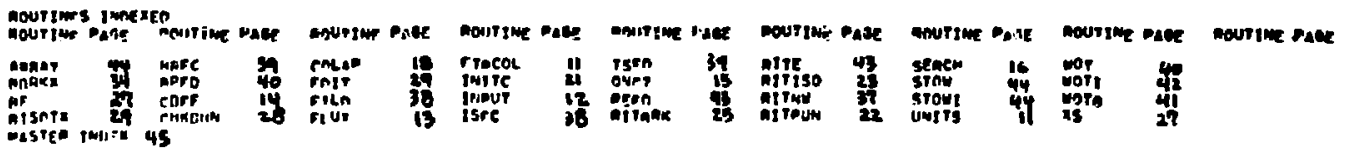


FINE GROUP FLUMES USED IN COLLAPSING

$6 R O$
1
2
3
5
5
6
7
8
20
11
12
13
14
15
28
17
18
19
20
21
22
23
24
25
26
27
28
29

PHI-FV I

4.03357E::7

1.76ri5E:=6

3. OTTARE W

3.27ก7LE $\bullet E$

$2.54113 E+\pi$

2.06909k.คĀ.

1.035?3E+:

1.03373E -F

2.0GPAAE - :

2.06969E* $=6$

2.0694>E०

2. 06ABAE IA

$1.9655 \mathrm{AE}$ : $:$

1.034AME * :

1.15939F: :

2.06779E + :

2. $069055 \cdot 50$

2.06RTAE -

$6.8951 \mathrm{~A}+27$

$6.89709 E+F 7$

$6.69575 E *=7$

2. 07 n $5 E$ tie

2. Q6AASE* *A

2. DGA9AE \& $\mathrm{B}$

2.06F5SE:-

3.1033uE $\div=$

3.104B3E*:

6.20B SAE : $=$

$1.26144 E .=0$

COABSE GROLP FLJX VALUES USEN IN COLLAPSE GROUP PHIEE 1

1.42174E +8

7.2613150

5. 26131ट.28

S. 357\%वE.

2.89 sS $\theta=0$

PAOCESSzNG ISnTOPE NHME 
IAPLT VALLE FON VERSTON ITt CCEC INTERFACE FILES KIPR $T$ misorm Gingot? nDERT ENDF JB

MALT 1165

EFIS5

ECART Gi

PRGCESSING ISCTONE RAME C I145

INPLT VALWE FOH VERSION II CCCE INTERFACE FILES

hITR

nIOENT ENOFHT

HWT 1165

EFISS $\quad-0$.

PAOCESSING ISnTOPE NAME O 1134

IAPUT VALUE FOH VERSION IIJ CCCC INTEARACE FILES xHA 1

n?SONA OIsO5?

nIDENT EROFHB

MUAT 1130

EFISS $\quad-0$.

PROCESSING ISOTCPE HAME NAIIS6

inPLIT VALUE FOR VEASION III CCCC INTERFACE FILES

KTR

niEENT ENDFH3

huAT 1156

EFISS

PRCCESSING ISOTUNE Hame COII2I

INPUT VALUE FGR VERSTON III CCCC INTEAFACE FILES KAR 5

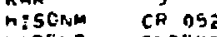

MIOENT ENDFHJ

HMAT 122

EFIS5

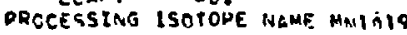

INPUT VALUE FOR VERSION III CCCC INTERFACE FILES KAT 5

KHSONM MN OSE

HIOENT ENOFH

HMAT 1010

EFISS -O.

PROCESSTI G ISTTOHE NAME FFIIRA

INPUT VALUE FOR VERSION TII CCCE INTERFACE FILES $K H{ }^{\circ}$

MISONM FE OSE

HTOENT ENUFA3

HMAT 1180

EFIS5 -0.

ECAPT -0 .

PACEESSING ISOYOPE NAME NTIIZ3

IHPUT VALUE GOA VEASION III CCCC INTERFACE FILES KSP 5

NisCivin AI 052

HIDENT ENUFB?

HMAT 1123

EFISS $\quad-0$.

ECAPT 0 ,

DROCESSING ISOTOHE NaHE MAIII)

thPUT VALUE FOR VeRSION III CCCC INTEAFACE FILES KRR 5

MISONH MO ASE

MIOENT ENUFBS

HMAT IIII

EFISS -0 .

ECAPT ISOT'

PROCESSING ISOTLDE NAME U 1197

InPUT VALUE For VERSION TII CCCC INTERFACE FILES KAP

HISONM US 052

HIDTT 1157

EFISS $\quad 3.20000 E-1$

ECAPT

PAOCESSING ISOTOPE NAME U IIFB
INPUT VALUE FOR VERSION III CCCC INTERFACE FILES KAR

HISONM UO OSF

KMAT 1158

EFISS 3.20000E-2T

ECAPT 0 .

PROCESSING ISOTOPE NAME PIIIISO

INPUT VALUE FOR VEASION iII CCCC INTERFACE FILES KGR

MYSONM PUPOS?

HIDENT ENOFHS

HMAT 1150

EFISS 3,2000ne-1i

ECAPT 0.

PRocessing Isotope name puitís

INPUT VALUE FOF VEASION III CCCC INTERFACE FILES KBP 1 HISONH PUDOS? HIOENT ENUFH3

HMAT 1105

EFISS 3,2nONOE-1T

ECAPT $\rightarrow$.

PROCESSINO zSOTOPE NaME Pillíg

INPUT VALỤE FCQ VERSION III CCCC INTERFACE FILES KBR 1

HISONH PUIO5Z

HIDENT ENOFBS

HMAT 1106

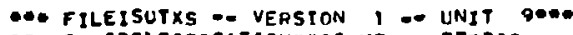

- UUSER IDENTIFTCATIONH HSIMMER FTR300

FILE CONTROL PARAMETFAS

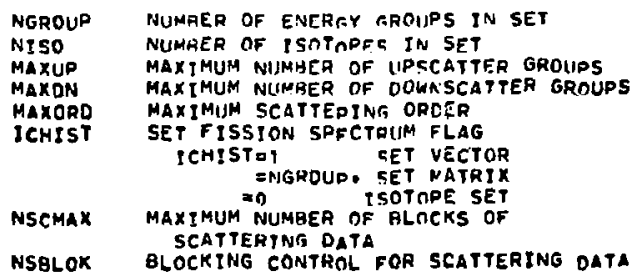

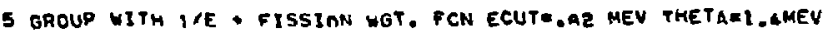

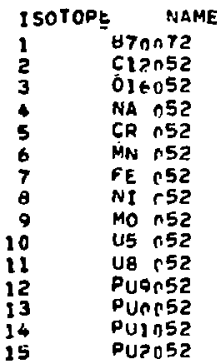

MEAN NEUTRON VELOCTTY IN AROUP I

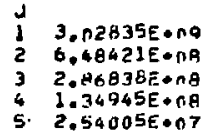

MAXIHIIM ENERGY HOUND

GROUP

$$
\begin{array}{ll}
1 & 1.00000 E+0.7 \\
2 & 4.97900 E+0.5 \\
3 & 1.11100 E+05 \\
4 & 2.55400 E+04 \\
5 & 1.23400 E+03
\end{array}
$$

MINIMUM ENẸRGY HOUND OF SFT

$$
\text { - GAPBAE +ZÁ }
$$

NUMAER OF HECNRDS TO RE SKIPPEO

ISOTOP NUMBER I 


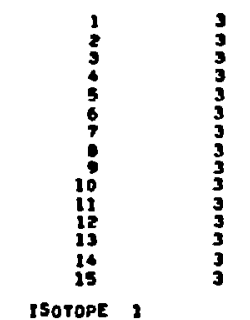

ISOTOPE CONTROL PAGANETERE

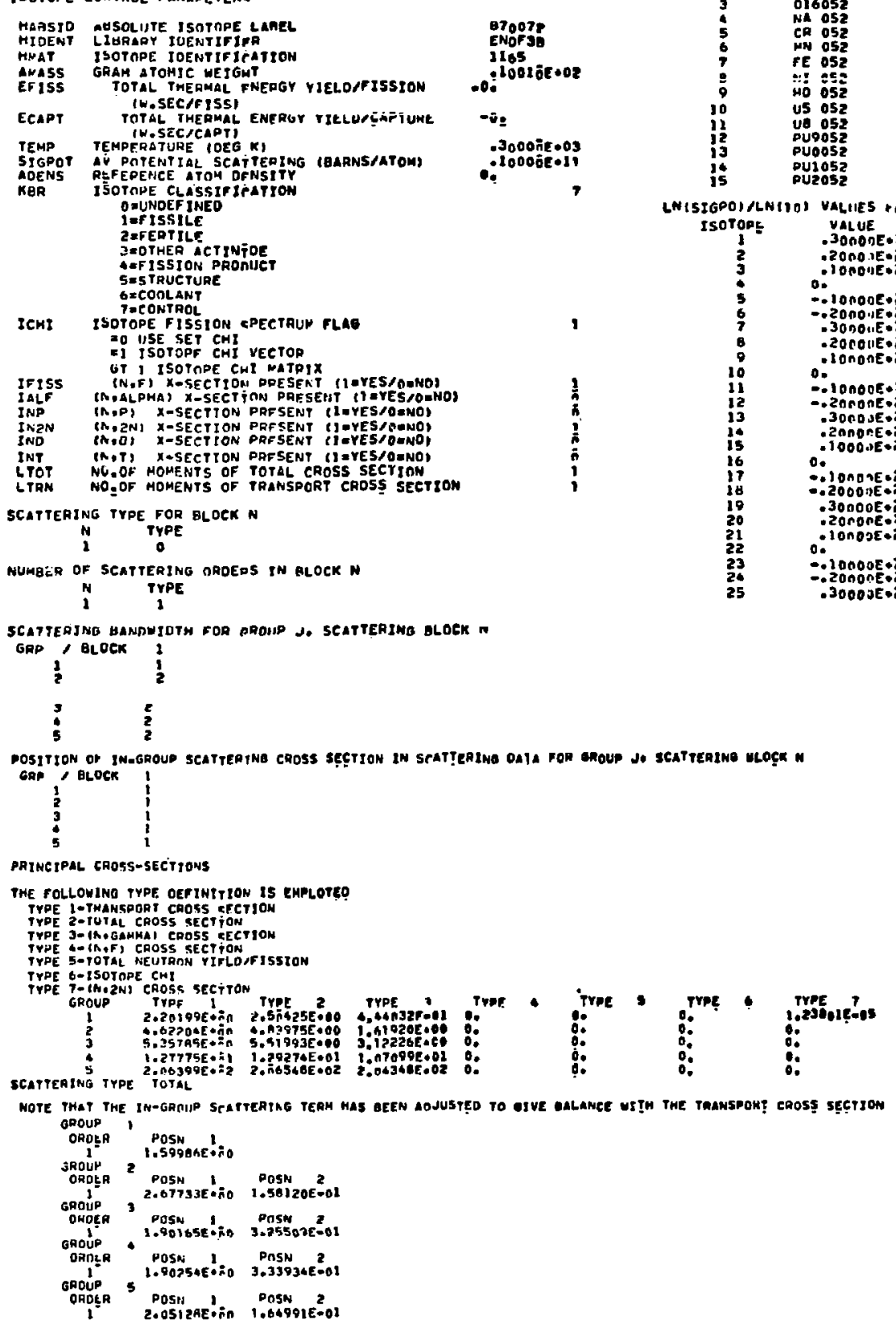

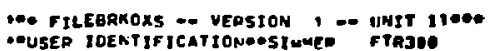

FILE CONTROL DARAMETERS

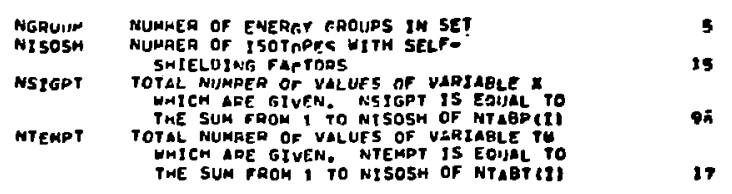

ISOTOPE NAME

R70072

c.2052
016052

:

us 05

pupose

puoss

VALIES +OP ALL ISOTOPES

$2000.1 E \bullet$ ก

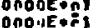

(O)

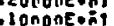

年

OOJE CEI

orone:

o lonogent

3000JEकTि 


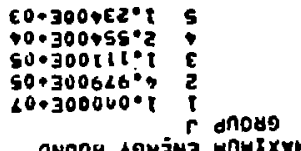

onnog agyins wกw

\begin{tabular}{|c|}
\hline 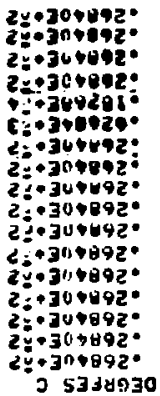 \\
\hline
\end{tabular}

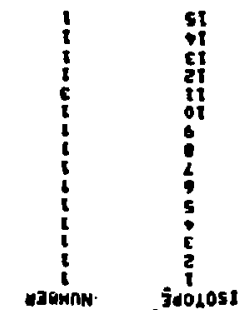

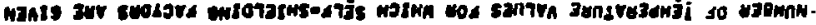

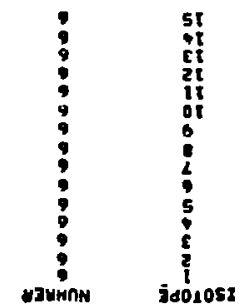

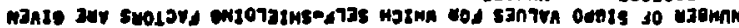

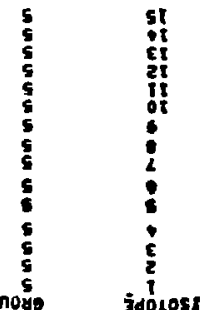

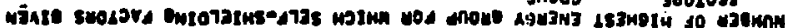

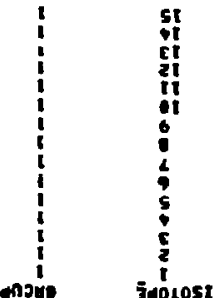

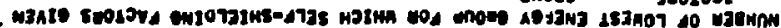

$0: 309<89 *$

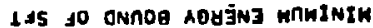

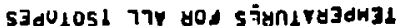

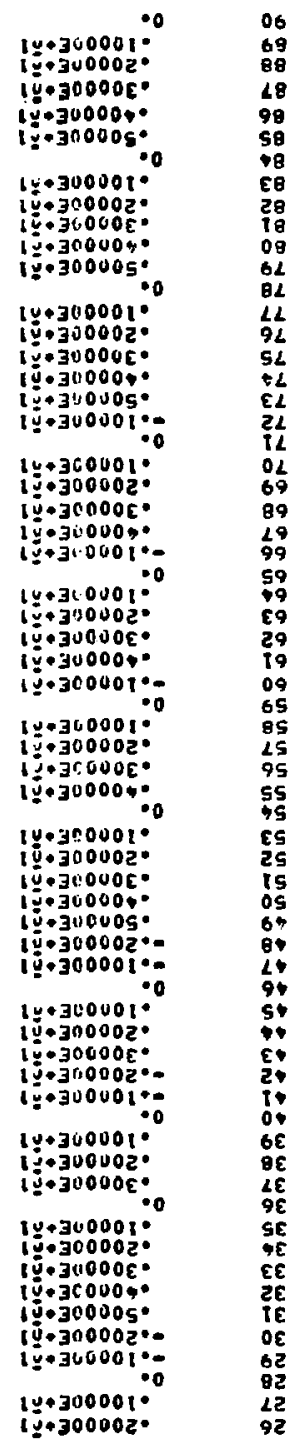


CDIT FOA ISOTOPE NAHEO GTÓT? TOLLCUING IS THE TAFLE INOEX

$J$ GROUP NUMAEP

- TEHotaAtIRE NumaE

M SIGPO NUMHER

TOTAL SELF-SMIELOING ACTARS

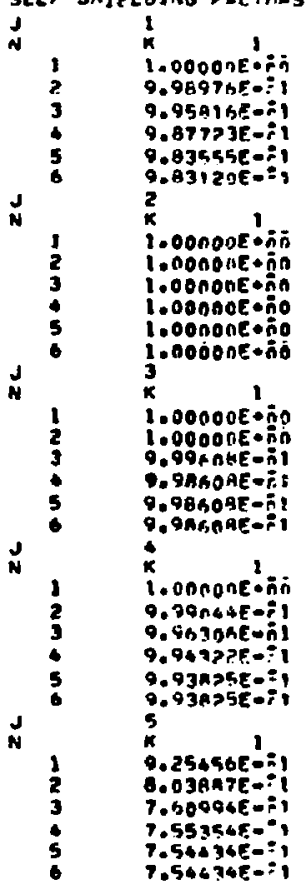

CAPTURE SELF-SMIFLOJMG FAPTADS

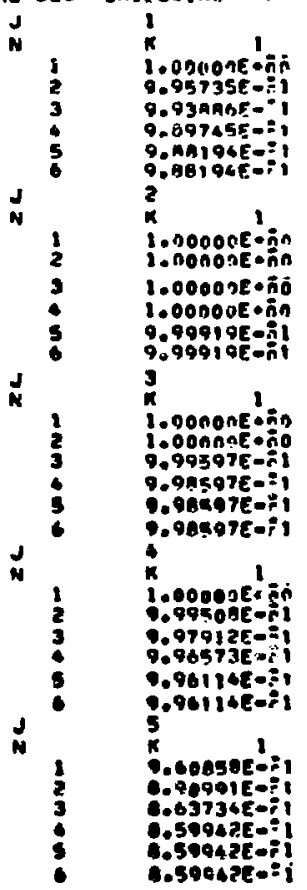

ISOTODE $\quad$

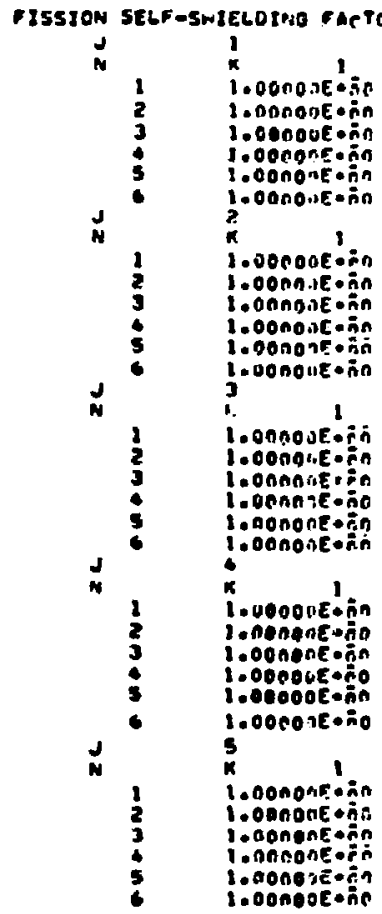

ThansPoAT SELrosnlecotno gactons

isntape

Isoropt I

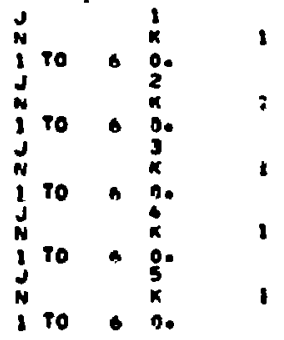

Elastic selfosnieloing fartons

ISOROAE 1

Isorock 1

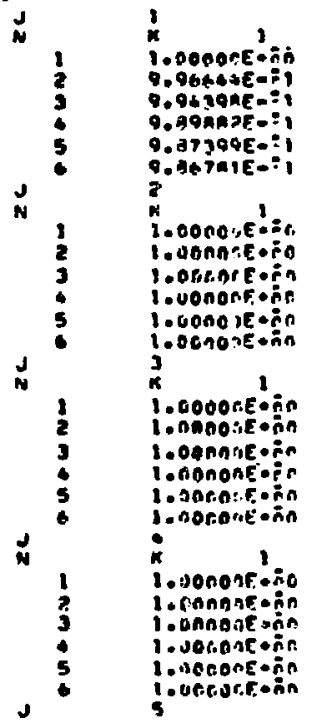


*

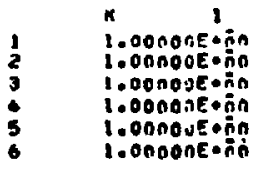

choss setrions

positron ceuss section

Poignital seatreativo

INELLATIE

Elastic

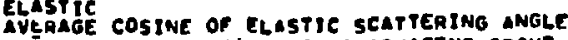

ELASTIC OOMNOSCATTERTNG TO ADJACENT GAOUP

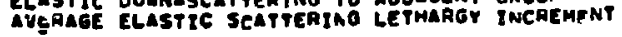

onoup

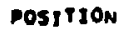

POSITION

\begin{tabular}{|c|}
\hline $\begin{array}{l}0 \\
0 \\
0 \\
0\end{array}$ \\
\hline
\end{tabular}

3.31N6SE-O2 2.02703E.06

$1.49112 E-01 \quad 1.57867 E-01 \quad 1.72125 E-01$

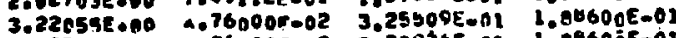
$2.39769 E+A$ A.76000E-0? 3.33934E-01 1.8060ñE-0I

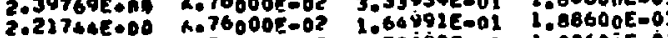
: $2.39769 E+114$ a $2.20000 \mathrm{~F}+00$

$4.76000 E-0 ? \quad 3.55 j 89 E-01$. 1.8660óE-OL 